\title{
INTERPRETAÇÃO ECONÔMICA DE ENSAIOS DE ADUBAÇÃO DE MILHO EM PERNAMBUCO
}

\author{
ISRAEL. PEREIRA DA SILVA
}

Engenheiro-Agrônomo

\author{
Orientador: Dr. Frederico Pimentel Gomes
}

\begin{abstract}
Dissertação apresentada à Escola Superior de Agricultura "Luiz de Queiroz", da Universidade de São Paulo, para obłenção do título de Mestre em Experimentação e Estatística.
\end{abstract}

PIRACICA B A

Estado de São Paulo - Brasil

Junho, 1978 
ii。

Ao Prof. Märio Coetho de Andrade Lima (in memorian)

A meus pais

A minha esposa

DEDICO 


\section{AGRADECIMENTOS}

Ao Professor Dr. Frederico Pimentel Gomes, pela firme orientação prestada a este trabalho, como também pela versão para a lingua inglesa do resumo.

A Universidade Federal Rural de Pernambuco, pela opor tunidade oferecida para realização do curso de pós-graduação.

Ao Instituto de Pesquisas Agronômicas de Pernambuco (IPA) e à Associação Nacional para Difusão de Adubos (ANDA), pelo fornecimento dos dados.

Ao Plano CAPES-PICD e ao PEAS, pela bolsa de estudos concedida.

Ao Professor Dr. Humberto de Campos, pelas valiosas sugestões。

Aos Professores do Departamento de Matemática e Esta tística da Escola Superior de Agricultura "Luiz de Queiroz", pela perfeição dos ensinamentos recebidos.

Aos colegas do curso de pós-graduação, pelo espírito de solidariedade e companheirismo.

Ao Eng ${ }^{8}-A_{g}{ }^{\circledR}$ Nilo Sérgio de Carvalho Pereira, pela valiosa colaboração.

Ao Professor Milton Tavares Bezerra de Melo, Chefe do Departamento de Física e Matemática da UFR-PE, pelo espirito de colaboração e despreendimento com que nos incentivou.

A Srta. Maria Izalina Ferreira Alves, Secretária do Departamento de Matemática e Estatística da ESALQ, pelos trabalhos datilográficos.

A todos que, direta ou indiretamente, colaboraram pa ra a confecção do presente trabalho. 
I $N D I C E$

Pảg.

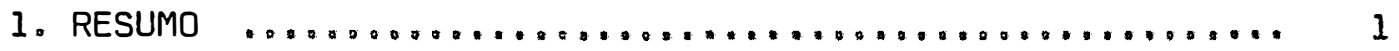

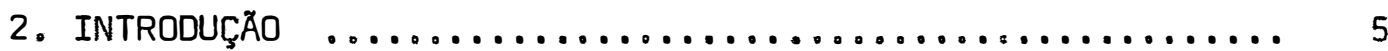

3. REVISÃo DE LITERATURA $\ldots \ldots \ldots \ldots \ldots \ldots \ldots \ldots \ldots \ldots \ldots \ldots \ldots \ldots \ldots \ldots \ldots \ldots$

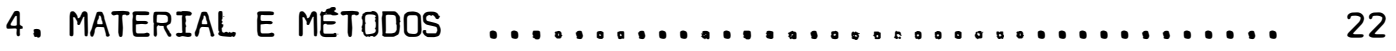

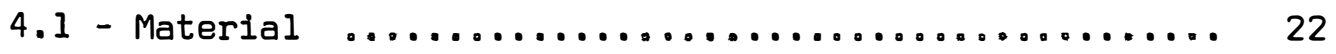

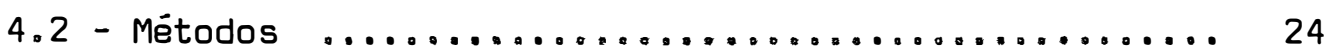

4.2 .1 - Os grupos de ensaios ................ 24

4.2.1.1 - Grupos de ensaios fatoriais .... 25

4.2.1.2 - Grupos dos tratamentos adicionais 26

4.2 .2 - Esquemas estatisticos $\ldots \ldots \ldots \ldots \ldots \ldots \ldots 26$

4.2 .3 - Estudo do modelo polinomial quadrático .... 30

4.2.3.1 - Estimativa dos parâmetros e seus respectivos intervalos de confian-

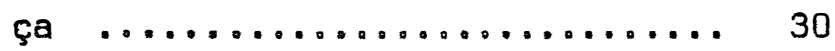

4.2.3.2 - Intervalos de confiança das estima tivas dos rendimentos ........ 37

4.2.3.3 - Doses ötimas dos fatores $x_{1}, x_{2} e$ $x_{3}$ obtidas através da superfície de resposta $\ldots \ldots \ldots \ldots \ldots . . . . . .40$

4.2 .3 .4 - Cortes da superfície ........ 42

4.2.3.5 - Análise da regressão e coeficientes de determinação total da superfície de resposta $\ldots \ldots \ldots \ldots \ldots \ldots .45$ 
Päg。

4.2 .4 - 0 trinômio do segundo grau $\ldots \ldots \ldots \ldots \ldots \ldots . . \ldots 1$

4.2.4.1 - Estimativa dos parâmetros e de seus respectivos intervalos de confiança 51

4.2.4.2 - As doses econômicas e seus intervalos de confiança $\ldots \ldots \ldots \ldots \ldots . . . . .45$

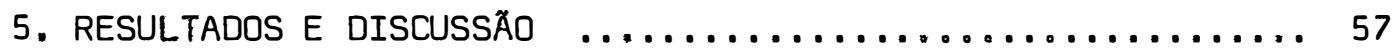

5.1 - O Ajustamento da Regressão Polinomial Quadrätica ... 59

5.1.1 - Resultados e discussão dos parâmetros e seus respectivos intervalos de confiança ...... 59

5.1 .2 - Os intervalos de confiança das estimativas dos rendimentos .........................61

5.1 .3 - As doses ótimas de $\mathrm{N}, \mathrm{P}_{2} \mathrm{O}_{5}$ e $\mathrm{K}_{2} \mathrm{O}$ e discussão do máximo $\ldots \ldots \ldots \ldots \ldots \ldots \ldots \ldots . . \ldots \ldots$

5.1 .4 - Resultados e discussão dos cortes dados na superficie ....................... 66

5.1.5 - Valores de $F$ das regressões e dos desvios das regressões e coeficientes de determinação to-

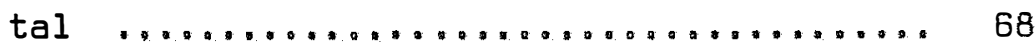

5.2 - 0 Uso do Trinômio do Segundo Grau ............. 70

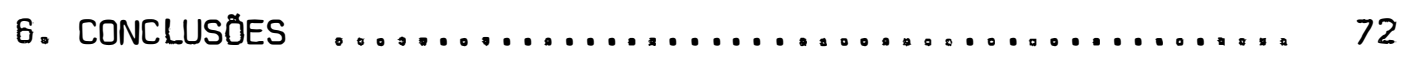

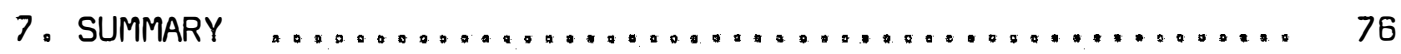

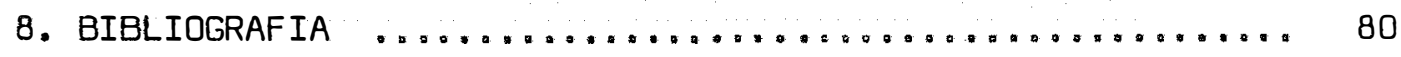

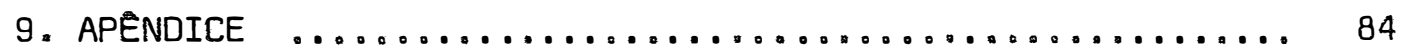




\section{RESUMO}

Com a finalidade de obter resultados que servissem de base à recomendação económica de fórmulas de adubação para a cultura do milho no Estado de Pernambuco, Brasil, dispomos de 37 ensaios fatoriais $3^{3}$ de $N$ 。 P e K, com duas repetições e 34 em blocos casualizados, com sels repetiçōes, onde estudou-se o calcário, também em 3 níveis.

As recomendações apresentadas neste trabalho foram com base em 9 grupos de ensaios, abrangendo 14 municípios e 3 regiōes fisiográficas, nitidamente distintas.

Para os ensaios fatoriais, foi ajustado o modelo de regressäo polinomial quadrätica:

$$
\begin{aligned}
& y=a_{0}+a_{11} x_{1}^{2}+a_{22} x_{2}^{2}+a_{33} x_{3}^{2}+a_{12} x_{1} x_{2}+a_{13} x_{1} x_{3}+a_{23} x_{2} x_{3}+a_{14} x_{1}+ \\
& +a_{24} x_{2}+a_{34} x_{3}
\end{aligned}
$$


onde foram abordados os seguintes aspectos:

1 - Estimativas, intervalos de confiança e teste t dos parâme tros.

2 - Intervalos de confiança das estimativas dos rendimentos.

3 - Doses ötimas de $\mathrm{N}, \mathrm{P}_{2} \mathrm{O}_{5}$ e $\mathrm{K}_{2} \mathrm{O}$ da superfície de resposta.

4 - Comportamento da superfície ao se fixar duas de suas variá veis em suas doses médias.

5 - O ajustamento com base na análise da regressão e no coeficiente de determinação total.

Na determinação das doses econōmicas para o calcário, utilizou-se o trinōmio do $2^{\circ}$ grau.

As principais conclusões obtidas neste trabalho foram:

1 - Os parâmetros da regressão polinomial quadrática, para os grupos de ensaios, apresentaram intervalos de confiança bastante am. plos e até absurdos, em alguns casos. Essa imprecisão reflete-se na estimativa dos rendimentos, embora menos acentuadamente.

2 - A não significância dos parâmetros das interações sugere o estudo da superfície, desprezando-os.

3 - Com exceção dos grupos 1 e 8, o ajustamento da superfície de resposta aos dados pode ser considerado como razoável.

4 - Os cortes da superfície indicaram como economicamente acon selháveis as seguintes quantidades de $\mathrm{N}, \mathrm{P}_{2} \mathrm{O}_{5}$ e $\mathrm{K}_{2} \mathrm{O}$, em kg/ha: 


\begin{tabular}{|c|c|c|c|}
\hline Grupos & $\begin{array}{c}N \\
(\mathrm{~kg} / \mathrm{ha})\end{array}$ & ${ }_{(\mathrm{kg}}^{\mathrm{P}_{2} \mathrm{O}_{5}}$ & $\begin{array}{c}\mathrm{K}_{2}{ }^{0} \\
\left(\mathrm{~kg}{ }_{\mathrm{ha}}\right)\end{array}$ \\
\hline 1 & minimo & 17,3 & mínimo \\
\hline 2 & $\cdots$ & 34,2 & zero \\
\hline 3 & 51,6 & zero & mínimo \\
\hline 4 & 71,0 & 69,7 & 29,0 \\
\hline 5 & 73,6 & 54,3 & minimo \\
\hline 6 & 20,1 & 33,4 & minimo \\
\hline 7 & minimo & zero & minimo \\
\hline 8 & 33,7 & mínimo & minimo \\
\hline 9 & 96,5 & mínimo & 29,2 \\
\hline
\end{tabular}

Obs.:A linha pontilhada significa que a dose obtida extrapolou o in tervalo pesquisado.

5 - Intervalos de confiança para a dose economicamente recomen dável, calculados em casos em que o coeficiente do termo quadrätico näo diferiu significativamente de zero, deram resultados absurdos,o que comprova ser esse cälculo desaconselhävel nessas circunstânclas.

6 - Para os grupos $1,3,4$ \& 6, é recomendada a aplicaçäo de $1000 \mathrm{~kg} / \mathrm{ha}$ de calcärio, e para o grupo 2 a calagem não é recomendada. Os demais grupos apresentaram ponto de mínimo, e não de máximo.

7 - A obtenção do máximo, através da regressão polinomial quadrática,torna-se problemática quando os grupos são constituídos de poucos ensaios, e suas precisões delxam muito a desejar, como foi o nosso caso. 
8 - As recomendações feitas neste trabalho näo merecem muita confiança, em virtude do pequeno número de ensaios de que se dispós. não devendo, portanto, serem encaradas como definitivas. Entretanto, dão uma boa indicação para trabalhos subsequentes. 
5.

\section{INTRODUÇĀO}

Analisando a sltuaçāa da cultura do milho em Pernambuco, depara-se com um quadro näo multo animador. Segundo o Anuärio Estatistico do Instituto Brasileiro de Geografia e Estatística, de 1976, a situação dos três últimos anos, publicados por essa entidade para o Estado de Pernambuco, em quantidade de milho em grão, è a seguinte:

\begin{tabular}{cccc}
\hline Anos & $\begin{array}{c}\text { Area } \\
\text { Colhida } \\
\text { (ha) }\end{array}$ & $\begin{array}{c}\text { Quantidade } \\
\text { Produzida } \\
(\mathrm{t})\end{array}$ & $\begin{array}{c}\text { Rendimento } \\
\text { Médio } \\
(\mathrm{kg} / \mathrm{ha})\end{array}$ \\
\hline 1974 & 520.000 & 405.600 & 780 \\
1975 & 483.000 & 352.685 & 730 \\
1976 & 300.000 & 165.000 & 550 \\
\hline
\end{tabular}


Observa-se, portanto, que não só caiu nesses três úl timos anos a produção, em decorrêncla de menores áreas cultivadas , como também a produtividade. Por outro lado, em relação aos demais Estados brasileiros, Pernambuco que em 1974 ocupava o oitavo lugar em produção e o décimo em produtividade, calu em 1976 para décimo primeiro e décimo quinto respectivamente.

E claro que muitos fatores estão envolvidos no processo, não sendo o problema especificamente econômico. Entretanto, não há dúvidas de que, ao lado de outras técnicas, a adubação recomendada racionalmente possa melhorar sensivelmente esse quadro. Nes se sentido, além de outras culturas, o milho vem sendo estudado pelo Instituto de Pesquisas Agronômicas (IPA) sob vários aspectos e, nesses ültimos anos, a pesquisa tem sido dirigida principalmente no sentido de verificar as respostas de elementos essenciais à planta com a finalidade de melhorar o rendimento cultural. Em 1972, ènfase maior fol dada ao estudo, com a celebração do convênio entre esse órgão de pesquisa e a Associação Nacional Para Difusão de Adubos (ANDA). A finalidade do convênio sería a de estudar as respostas das principais culturas do Estado aos macronutrientes $N, P$ e $K$ e ao cál cário nas regiöes proplcias às culturas. Para isso, foram lançados vários ensaios em 3 anos consecutivos e, com base nos resultados ob tidos, partiu-se para a racionalização do uso desses insumos. Este material, convenientemente trabalhado, poderia servir como ponto de partida para o estudo econômico da aplicação 
desses insumos, estudo esse possível através das chamadas "funções de produção", das quals a superfície polinomial quadrática e o trinômio do $2^{9}$ grau, além da Lei de Mitscherlich, são por demais usados, quando se trata de ajustamento a dados de ensaios de adubação. 
8.

\section{REVISÃO DE LITERATURA}

HEADY e PESEK (1954) apresentaram um estudo sobre cin co diferentes tipos de funções de produção, ajustadas a um ensaio de adubação $N$ e $P$, com níveis variando de 0 a 320 lb/acre, num total de 57 combinações de tratamentos, com a cultura do milho, no Des te de Iowa. Os autores ajustaram um total de 35 equações, sendo apresentadas apenas 5 delas. Para essas cinco funções, foram calcula dos o valor do teste $t$ para os parâmetros e, também, os coeficientes de correlação, chegando à conclusão de que a melhor equação que representava os dados era:

$$
\begin{array}{r}
\hat{Y}=5,682-0,316 \mathrm{~N}-0,147 \mathrm{P}+6,3512 \sqrt{\mathrm{N}}+8,5155 \sqrt{P}+0,3410 \sqrt{\mathrm{NP}} . \\
0 \text { máximo dessa função fol obtido com as doses de } \\
397,6 \mathrm{lb} / \text { acre e } 336,6 \mathrm{lb} / \text { acre de } \mathrm{N} \text { e } \mathrm{P}_{2} \mathrm{O}_{5} \text {, respectivamente, valores }
\end{array}
$$


esses que extrapolaram as doses ensaiadas.

ZAGATTO \& PIMENTEL GOMES (1960) discutiram, sob o pon to de vista econōmico, o uso de algumas funções de produção, tadas a uma variável, notadamente a equação de Mitscherlich e o trinômio do $2^{\circ}$ grau, procurando tornar máxima a função:

$$
z=\frac{R-C}{C}=\frac{R}{C}-1 \text {. }
$$

onde, $R=$ receita bruta;

$C=$ custo de produção.

Os autores preocuparam-se com o fato de que, sob con dições de capital limitado, o mais importante não é a obtenção da produção máxima e sım, o mais alto retorno por cruzeiro dispendido. PIMENTEL GOMES e CAMPOS (1966), trabalhando com ensaios individuais e com grupos de ensaios de adubação de milho, em vários Estados brasileiros, verificaram a predominâncla do efelto de nitrogênio nos diversos tipos de clima e solos em que foram conduzidos os experimentos. Em segundo lugar, colocou-se o fósforo com numerosos casos favoráveis, notadamente no Triângulo Mineiro e Goiàs, enquanto que o potässio, na maioria dos casos, não apresentou efeito significativo. Quanto ao calcário, verificaram também que, em so los ácidos, especialmente nos cerrados, a calagem é favorável e até indispensável em alguns casos.

Os autores determinaram também as doses economicamen te aconselhäveis de nutrientes, para as regiões estudadas, pela Lei 
de Mitscherlich, comparando esses resultados com os obtidos com uma superfície polinomial do $2^{9}$ grau a três variáveis independentes. No caso particular de Pernambuco, recomendaram para a Região do Vale do São Francisco doses de nitrogênio que variaram de 70 a $80 \mathrm{~kg} / \mathrm{ha}$. CAMPOS (1967), utilizando 50 ensaios fatoriais de adubação $3^{3}$ com a cultura de milho na região de Ribeirão Preto (SP), em terra roxa legitima, ajustou o modelo:

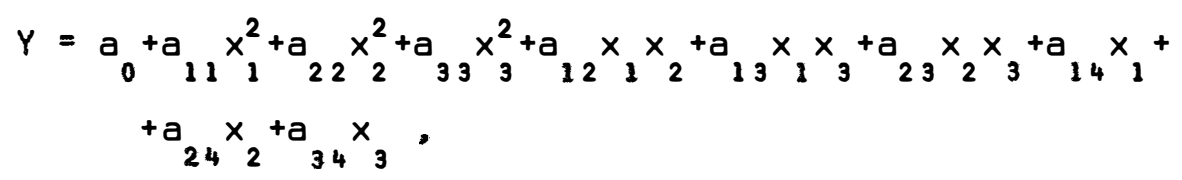

aos referidos dados, preocupando-se em determinar as doses economicamente aconselháveis para $\mathrm{N}, \mathrm{P}_{2} \mathrm{O}_{5}$ e $\mathrm{K}_{2} \mathrm{O}$, apōs a diagonalização da matriz M das derivadas parciais de segunda rordem.

O autor também apresentou um estudo dos cortes na su perfície, fixando duas das três variáveis independentes envolvidas no modelo, em suas doses padrão, bem como um estudo comparativo entre o delineamento rotativo de Box e o fatorial de $3^{3}$, concluindo que, equiparando-se os níveis de nutrientes através da redução à mesma escala e introduzindo-se um fator de correção para as variâncias das estimativas dos parâmetros provenientes do delineamento de Box, os fatoriais $3^{3}$ apresentam maior eficiência.

Quanto ao estudo da superfície, especificamente, o autor tirou uma série de conclusōes, cujas principais são: (a) as estimativas dos parâmetros da equação de superfície de resposta,tan to nos ensaios individuais como nos grupos de ensaios, possuem in- 
tervalos de confiança bastante amplos, traduzindo consequentemente uma imprecisão nas estimativas dos rendimentos, não sendo descartada, portanto, a possibilidade de estimativas negativas para a produ ção; (b) dos 50 ensaios individuais estudados, apenas 7 deram, de fato, uma dose economicamente aconsel hävel de nutriente, surgindo inclusive, um caso da função de receita líquida possuir mínimo. Hou ve, portanto, uma predominància de pontos de sela nos ensaios individuais; (c) há maior tendência de aparecimento de pontos de máximo na função de receita líquida, à medida que se procede ao agrupamento dos ensaios; (d) o estudo da superfície, através dos cortes, revelaram a mesma imprecisão de resultados que a superfície como um todo. Esses cortes ilustram bem o comportamento da suferfície, sob o ponto de vista da dose econômica: (e) a aplicação da regressão polinomial aos ensaios fatoriais $3^{3}$ de adubação se recomenda apenas para grupos de ensaios de boa precisão.

ZAGATTO e PIMENTEL GOMES (1967), estudando as funções de produção a uma, duas e três variáveis independentes, sob o ponto de vista económico, em fatoriais $3^{3}$, discutiram os resultados obtidos na determinação da dose econômica para a função quadrätica, Lei de Mitscherlich e superfície polinomial quadrática do $2^{\circ}$ grau, apre sentando algumas dificuldades que poderiam surgir, como também, algumas medidas para reduzir essas dificuldades, como:

1) Usar sempre grupos de ensaios numerosos ou ensaios isolados, com diversas repetições, e boa precisão. 
2) Não confiar em doses ótimas obtidas a partir de polinômios de coeficientes para os termos do segundo grau em $x_{1}^{2}, x_{2}^{2}, x_{3}^{2}$, etc. que não sejam significativamente diferentes de zero.

3) Verificar se os valores obtidos correspondem realmente a um mäximo.

4) Calcular intervalos de confiança para as doses ótimas encon tradas, para poder julgar o seu real valor.

MORAES (1969), com os dados de um ensaio de adubação NPK em milho, realizado na Venezuela, em delineamento em blocos casualizados com duas repetições, em esquema fatorial $3^{3}$ com níveis não equidistantes, ajustou-lhes uma superfície polinomial de respos ta a três variäveis independentes, representando as doses de $\mathrm{N}, \mathrm{P}_{2} \mathrm{O}_{5}$ e $K_{2} O$, concluindo, entre outras coisas, que:

a) a regressão polinomial obtida possuia intervalos de confian ça relativamente amplos para os parâmetros, razão pela qual os que apresentaram estimativas próximas de zero poderiam mudar de sinal dentro do intervalo de confiança;

b) as estimativas dos parámetros dos termos lineares diferiram

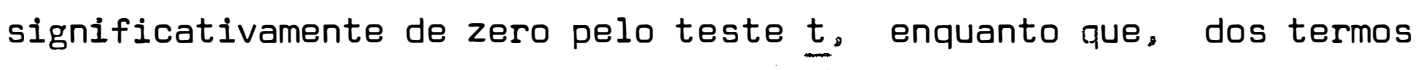
quadräticos, apenas o coeficiente $\hat{a}_{11}$, o fez, o que levou o autor a concluir que, dentro de intervalo considerado, a produção reage qua se linearmente aos nutrientes, sugerindo que o ensaio deveria ter doses mais elevadas, para que se pudesse constatar melhor oe efeitos dos coeficientes dos termos quadräticos: 
c) apesar de, tanto a regressão, como os desvios da regressão serem altamente significativos, o efeito do primeiro supera em muito o do segundo, sendo portanto satisfatória a regressão. Por outro lado, o coeficiente de determinação total da ordem de 0,949 , ou seja, 94,9\% da variação sendo explicada pela regressão, revela um bom ajustamento do modelo aos dados:

d) a função da receita líquida obtida, não possui máximo,e sim ponto de sela.

PIMENTEL GOMES (1969) chama atenção para o fato de que, a simples solução do sistema de equações, formado com as derivadas parciais de primeira ordem da função de receita líquida, não implica, necessariamente, na obtenção de máximo, sendo necessário, portanto, verificar se a matriz $\mathrm{B}$ das derivadas parciais de segunda ordem é definida negativa. O autor relacionou alguns trabalhos onde a não observação dessa condição implicou em interpretações errôneas dos resultados, e mostrou que, pelo fato dos coeficientes dos termos quadráticos serem todos negativos, não necessariamente se obtém um ponto de máximo, sendo preciso, portanto, a diagonalização e pos terior interpretação dos elementos dessa matriz, mesmo nesse caso. Todas essas observações foram feitas pelo autor ao estudar uma superfície polinomial de resposta a três variáveis inde pendentes, salientando ainda que, constatada a existência do máximo, é indicado testar os parâmetros dos termos quadráticos e aceitá-lo se estiver na região explorada pelo experimentador. 


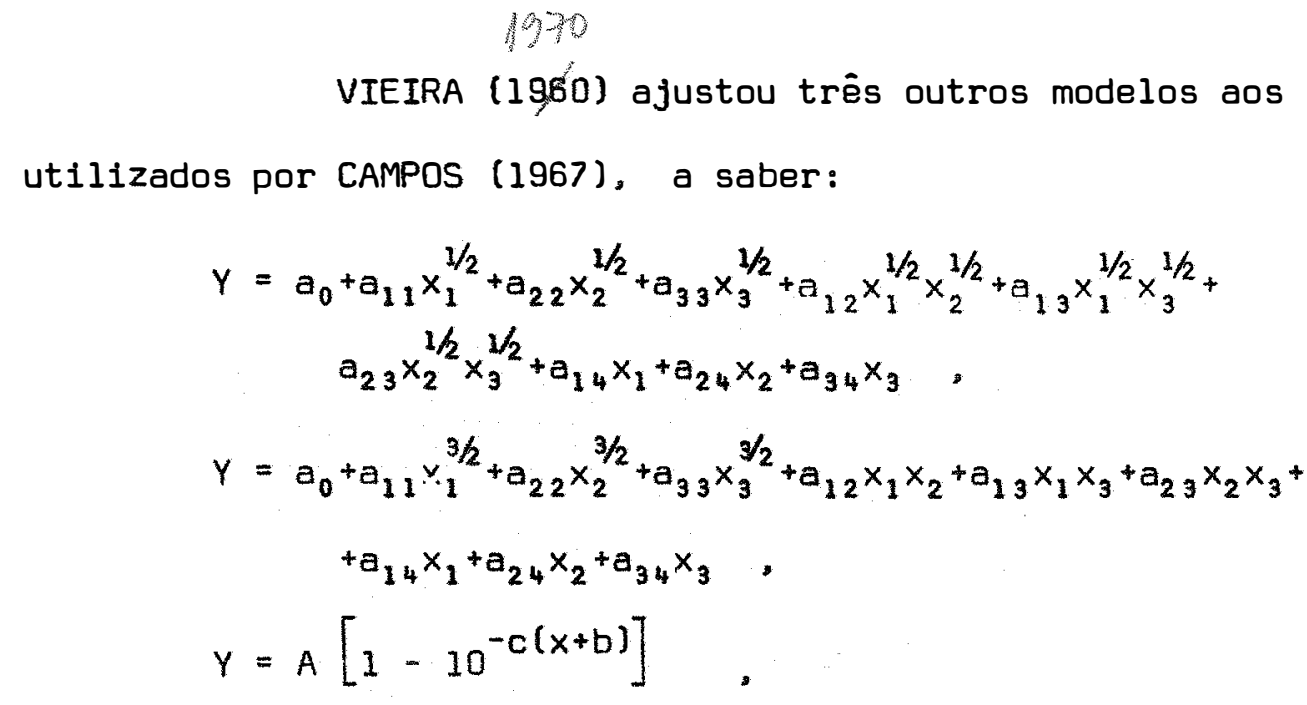

dando ènfase à comparação das doses economicamente aconselháveis de nutrientes, obtidas pelas quatro funções de produção, incluindo o modelo estudado por CAMPOS (1967) tirando, entre outras, as seguintes conclusões:

a) Dos três modelos polinomiais ajustados, dois revelaram-se praticamente iguais nos resultados: o modelo quadrático e o de grau 3/2, indicando, portanto, o uso do primeiro, por apresentar o de grau $3 / 2$ maiores dificuldades de cálculo, principalmente na solução do sistema de equações oriundas da função de receita líquida.

b) E recomendado o uso da Lei de Mitscherlich na determinação da dose econômica de nutrientes, no caso de 3 niveis, uma vez que leva a soluções satisfatörias, através de métodos matemáticos simples. Entretanto, no caso de existirem interações significativas de nutrientes, ou queda de produção, devidas à adubação excessiva, recomenda-se a aplicação de regressão polinomial, preferencialmente o modelo de raiz quadrada. 
c) A aplicação da regressão polinomial aos ensaios de adubação se recomenda apenas para grupos de ensaios cuja análise estatistica dos experimentos mostre efeitos quadráticos significativos, sugerin do a utilização de doses elevadas de nutrientes.

CAMPOS e ARAUJO (1971), com base nas produções médias de 50 ensaios fatoriais de adubação de milho na Região de Ribeirão Preto (SP), ajustaram-1hes as funçōes de produção:

$$
\begin{aligned}
y=a_{0} & +a_{11} x_{1}^{2}+a_{22} x_{2}^{2}+a_{33} x_{3}^{2}+a_{12} x_{1} x_{2}+a_{13} x_{1} x_{3}+a_{23} x_{2} x_{3}+a_{14} x_{1}+ \\
& +a_{24} x_{2}+a_{34} x_{3} .
\end{aligned}
$$

e,

$$
Y=a_{0}+a_{11} x_{1}^{2}+a_{22} x_{2}^{2}+a_{33} x_{3}^{2}+a_{14} x_{1}+a_{24} x_{2}+a_{34} x_{3}
$$

tirando uma série de conclusões, entre outras: (a) verificou-se que as estimativas dos parâmetros correspondentes às interações, não sen do significativas, dão suporte metodológico para o estudo da superficie desprezando-as, fato esse evidenciado por apresentarem, ambos os modelos, coeficiente de determinação de $38 \%$; (b) embora os inter valos de confiança dos parâmetros sejam relativamente amplos, há um estreiramento muito acentuado no caso dos rendimentos culturais. Es se estreitamento é mais acentuado, entretanto, no caso do modelo sem as interações, decorrentes das fórmulas que estimam as variâncias pa ra os dois casos. O importante, porém, é que, nos dois casos, as am plitudes dos intervalos foram sempre inferiores a $10 \%$ dos rendimentos estimados: (c) dos três nutrientes estudados, o fósforo foi o 
que se apresentou mais sensível às oscilações de preço. Considerando uma alternativa, o subsídio dos preços dos nutrientes básicos, por parte do Governo, atenção especial deveria ser dada ao $\mathrm{P}_{2} \mathrm{O}_{5}$, principalmente se uma grande percentagem da variação na taxa de retorno do capital empatado em adubação for explicada por variações no preço deste nutriente; (d) de uma maneira geral, observou-se que as doses económicas obtidas nos cortes da superfície, foram bastante próximas das obtidas na superfície.

WANDERLEY (1971) apresentou um estudo do comportamen to da cultura do milho em Pernambuco, quanto à adubação com $N, P, K$, Ca e micronutrientes, em 6 locais diferentes, concluindo que houve predominância do efeito de NPK. Em segundo lugar colocou-se o calcário, com resultados favoráveis em três dos seis locais estudados, não havendo resposta para a mistura de microelementos, com exceção de um único local. O autor salientou ainda que, só foi possivel estabelecer doses econômicas para dois dos seis locais estudados, mes mo assim com restrições. Finalmente, observou a falta de estrutura básica uniforme na experimentação com adubação de milho, o que prejudica, em grande parte, a obtenção de conclusōes gerais vālidas pa ra todo o Estado de Pernambuco.

OLIVEIRA (1974), comparando as precisões das estimativas dos parámetros dos coeficientes dos modelos do grupo fatorial $3^{k}$, para $k=2,3,4$, relativamente às estimativas correspondentes, obtidas dos modelos de delineamento composto central rotativo, para 
$k=2,3,4$, usando um ou mais pontos no centro, concluiu que, o gru po fatorial estudado é mais eficiente para o ajustamento de superfí cie de resposta, do que os delineamentos compostos centrais rotativos correspondentes.

VADLAMUDI e THIMM (1974), com base em dois ensaios de adubação de milho, realizados no Deste do Kenya, durante os anos de 1968 e 1969, ajustaram-lhes uma função de produção quadrática. inicialmente com 4 variáveis independentes $(N, P, K$ e D), sendo a última delas, representando a variação de 1968 até 1969. Essa variável assume o valor - 1 em 1968 e 1 em 1969. Quando $D=0$, a equação representa a função de produção média dos dois anos. 0 estudo dessa função levou os autores a suprimirem alguns termos, cujos coe ficientes apresentaram valor $\underline{t}$ muito baixo, não comprometendo, entretanto, a correlação entre os dados e o novo modelo, uma vez que não houve praticamente variação entre os dois coeficientes de deter minação, calculados para os dois casos.

Finalmente, tomando $D=0$ e fixando o valor de $k$,tam bém em zero, os autores obtiveram a seguinte função:

$$
\begin{aligned}
Y=36,271239 & +0,222608 N+0,206326 P-0,000496 N^{2}- \\
& -0,000880 P^{2}+0,000138 N P,
\end{aligned}
$$

com a qual foram determinadas as doses economicamente aconsel háveis de $N$ e $P$, considerando-se várias relações entre preços unitários dos nutrientes em relação ao produto. 
Os autores, examinando as relações de preços na época do trabalho, determinaram as quantidades de $173,52 \mathrm{~kg} / \mathrm{ha}$ e 105,24 $\mathrm{kg} / \mathrm{ha}$ de $\mathrm{N}$ e $\mathrm{P}$, respectivamente, como sendo as doses economicamente aconselháveis, o que daria uma receita líquida de Sh 886,62 por hec tare. Finalmente concluiram que, mesmo sob condições desfavoráveís, o uso de quantidades ótimas de N e P é recomendado para aumentar o lucro do produto em Kenya.

RAMIREZ DÅVILA e BENITEZ (1975), pesquisando 4 funções de produção, a saber:
1) Mitscherlich:$$
Y=A-B C^{X}
$$
2) Cobb-Douglas:$$
Y=A X^{B}
$$
3) Quadrática:$$
Y=A+B X+C X^{2}
$$
4) Raiz Quadrada:
$Y=A+B X+C X^{1 / 2}$,

quanto às vantagens e desvantagens de seu emprego, chegaram às seguintes conclusões:

a) No modelo de Mitscherlich, sendo assintótico ao valor $A$, o produto marginal nunca alcança valores negativos, indicadores do desequilíbrio biológico das plantas, causado por doses excessivas de fertilizantes, tendo, portanto, limitaçōes no seu uso.

b) 0 modelo de Cobb-Douglas permite somente, ou bem um constan te crescimento, ou decréscimo na produção marginal, não permitindo uma curva que inclua os três casos, não podendo, portanto, ser usada nos casos de existir produção marginal crescente e decrescente. 
c) 0 modelo polinomial quadrática pode ser usado com vantagens quando se trabalha com doses altas de nutrientes, pois permite mostrar possível diminuição na produção. Essa curva, por ser parabóli$c a$, é simétrica em relação ao valor máximo de $Y$, Na prätica, entretanto, a curva de resposta aos nutrientes mostra geralmente um pata mar amplo, onde a produção se estabiliza para logo ir decrescendo, devido às alterações fisiológicas provccadas por doses altas. Este decréscimo não guarda, entretanto, simetria com o crescimento.

d) 0 modelo de raiz quadrada é uma modificação do modelo polinomial quadrático, pertencente a uma familia de modelos que pode ser representada por:

$$
Y=A+B X^{d}+C X^{2 d}
$$

onde d é uma constante positiva.

CAMPOS \& PIMENTEL GOMES (1976) apresentam um interes sante estudo sobre as interaçōes lineares, $N^{\prime} \times P^{\prime}, N^{\prime} \times K^{\prime}$ e $P^{\prime} \times K^{\prime}$, em quatro grupos de ensaios fatoriais $3^{3}$ de adubação de milho, com $N$. $P$ e K, num total de 117 experimentos com 3.159 parcelas. Os autores concluiram, com grande generalidade, que, nesses tipos de ensaios, quando se consideram doses moderadas de nutrientes, as interações são de importáncia secundária e, na grande maioria dos casos, são de pequena monta, quando comparadas aos efeitos principais dos nutrientes. 
20 .

D'AULISIO (1976) determinou os intervalos de confian ça dos pontos de máximo ou de mínimo da função:

$$
P=\tilde{a}+6 P_{1}(x)+\hat{c} P_{2}(x)
$$

onde $P_{1}(x)$ e $P_{2}(x)$ são polinômios ortogonais de primeiro e segundo grau, respectivamente, por 4 processos distintos, a saber:

1) Método de Fieller:

2) Método empírico (por simulação):

3) Através de $V_{1}(x)$ (fórmula usual da variância):

4) Através de $V_{2}(x)$ (fórmula deduzida por D'AULISIO (1970).

A autora concluiu que o método de Fieller dá resulta dos absurdos para $\sigma^{2}>0,2500$. Em relação aos intervalos pelo método empírico, tomado como padrão, foram excessivamente amplos os dados por $V_{1}(x)$, e excessivamente curtos os obtidos a partir de $V_{2}(x)$. Dos 4 processos utilizados, os mais recomendáveis são os calculados pelo método empírico e a partir de $V_{2}(x)$, embora este seja um tanto curto.

COSTA (1977) apresenta um estudo de dois modelos polinomiais, sendo um deles o de raiz quadrada,ajustados a 27 ensaios fatoriais de adubação $3^{3}$, com a cultura do arroz, em diversas localidades do Estado de Goiás, concluindo que, tanto os valores de $F$ das regressões, como os coeficientes de determinação $R^{2}$, foram concordantes em ambos os modelos, em todos os casos estudados. As doses econômicas, obtidas nos cortes, foram bastante próximas das en- 
contradas nas superfícies, e seus intervalos de confiança muito amplos, dificultando a recomendação de adubação. 0 autor salientou ain da que, para a cultura do arroz, recomenda-se a aplicação das super fícies de resposta somente para grupos de ensaios de boa precisão. 


\section{MATERIAL E METODOS}

\section{1. - Material}

Os dados a serem utilizados neste trabalho foram gen tilmente cedidos pelo Instituto de Pesquisas Agronômicas do Estado de Pernambuco (IPA) e pela Associação Nacional para Difusão de Adubos (ANDA), e constam de ensaios fatoriais $3^{3}$ de adubação, com duas repetições e tratamentos adicionais. No esquema fatorial, onde os fa tores estudados foram nitrogênio, fösforo e potássio, houve o confundimento de dois graus de liberdade da interação tripla, sendo adotado, para todos eles, o grupo w de confundimento. 0 calcário foi estudado como tratamento adicional em blocos casualizados, com 6 re petições e em 3 nÍveis.

$$
\text { Estes ensaios foram lançados em Estações Experimen- }
$$
tais do IPA e em fazendas particulares, em locais proplcios à cultu 
ra, abrangendo de uma certa forma todas as regiōes fisiográficas do Estado de Pernambuco. Todos os experimentos utilizados neste trabatho foram de mesma estrutura, com as seguintes características:

a) Detalhes do ensaio:
a.1) Variedade:
Azteca
a.2) Espaçamento:
$1,00 \mathrm{~m} \times 0,40 \mathrm{~m}$
a.3) Área total:
$2.872 \mathrm{~m}^{2}$
a.4) Ârea ütil:
$1.296 \mathrm{~m}^{2}$

b) Detalhes da parcela:

b.1) Area total: $30 \mathrm{~m}^{2}$

b.2) Ârea ütil: $18 \mathrm{~m}^{2}$

b.3) Número de linhas:

b.4) Número de linhas úteis:

b.5) Número de covas por linha: 15

๖.6) Número de plantas: 150

b.7) Número de plantas úteis: 90

๖.8) Número de plantas por cova: 2

c) Níveis dos nutrientes (kg/ha):
c.l) Nitrogênio:
$0-50-100$
c.2) Fósforo:
D- $60-120$
c.3) Potássio:
$0-30-60$
c.4) Calcário:
$0-1000-2000$ 
d) Fonte dos nutrientes:
d.1) Nitrogênio:
Sulfato de amônio ( $20 \%$ de N)
d.2) Fósforo:
Superfosfato simples $\left(20 \%\right.$ de $\mathrm{P}_{2} \mathrm{O}_{5}$ )
d.3) Potássio:
Cloreto de potássio $\left(60 \%\right.$ de $\left.\mathrm{K}_{2}{ }^{0}\right)$
d.4) Calcário:
Calcário dolomítico ( $\mathrm{Ca}+\mathrm{Mg})$

e) Modo de aplicação dos adugos:

O fósforo, o potássio, o calcário e $30 \%$ de nitrogênio foram aplicados em fundação, e o restante do nitrogênio 30 dias após, em cobertura.

\section{2 - Mètodos}

\subsection{1 - Os grupos de ensaios}

Os grupos de ensaios, objetivo principal do nosso es tudo, com vistas à recomendação de doses econômicas dos nutrientes em questão, variaram de 2 a 6 experimentos, tanto para N, P e K, co mo para o calcário, apesar dos grupos serem, em alguns casos, cons tituídos de ensaios diferentes para ambos os casos.

CAMPOS (1967), ZAGATTO \& PIMENTEL GOMES (1967), VIEI RA (1970) e COSTA (1977) recomendam o uso de grupos de ensaios nume rosos e de boa precisão; entretanto, dada a heterogeneidade dos fatores climáticos e edáficos a que está submetido o Estado de Pernam buco, várias são as regiões consideradas fisiograficamente diferen- 
tes, o que torna problemático o agrupamento de maior nümero de ensaios. Além disso, um outro fator que contribuiu para o tamanho dos grupos foi a heterogeneidade das variāncias residuais que não permi tiu, em alguns casos, grupar ensaios de caracterfsticas semelhantes. Logo, na formaçäo dos grupos foram levados em conta os seguintes fa tores:
a) Regiões fisiogräficas;
b) Tipos de solos e vegetação;
c) Quadrados médios residuais.

\subsubsection{1 - Grupos dos ensaios fatoriais}

\begin{tabular}{|c|c|c|c|}
\hline Grupos & Municípios & $N^{2}$ de ensatos & Anos agrícolas \\
\hline 1 & Vitória de Santo Antão & 2 & 73,74 \\
\hline \multirow[t]{2}{*}{2} & Glória do Goitä & 2 & 72.73 \\
\hline & Limoeiro & 3 & $72,73,74$ \\
\hline \multirow[t]{2}{*}{3} & Surubim & 3 & $72,73,74$ \\
\hline & Vertentes & 3 & $72,73,74$ \\
\hline \multirow[t]{2}{*}{4} & Timbaúba & 2 & 72,74 \\
\hline & Vicência & 2 & 72,73 \\
\hline 5 & Caruaru & 5 & $72,73,74$ \\
\hline \multirow[t]{2}{*}{8} & Bom Conselho & 3 & $72,73,74$ \\
\hline & Garanhuns & 2 & 72,73 \\
\hline 7 & Serra Talhada & 2 & 73,74 \\
\hline \multirow[t]{2}{*}{8} & Belém do São Francisco & 3 & 73,74 \\
\hline & Petrolândia & 2 & 72,74 \\
\hline 9 & Tacaratu & 3 & $72,73,74$ \\
\hline
\end{tabular}




\subsubsection{2 - Grupos dos tratamentos adicionais}

\begin{tabular}{|c|c|c|c|}
\hline Grupos & Municipios & $N^{8}$ de ensaios & Anos agricolas \\
\hline 1 & Vitōria de Santo Antäo & 3 & $72,73,74$ \\
\hline \multirow[t]{2}{*}{2} & Glörla do Goitá & 3 & $72,73,74$ \\
\hline & Limoeiro & 3 & $72,73,74$ \\
\hline \multirow[t]{2}{*}{3} & Surubim & 3 & $72,73,74$ \\
\hline & Vertentes & 2 & 72,74 \\
\hline \multirow[t]{2}{*}{4} & Timbauba & 3 & $72,73,74$ \\
\hline & Vicência & 2 & 73,74 \\
\hline 5 & Caruaru & 4 & $72,73,74$ \\
\hline \multirow[t]{2}{*}{6} & Bom Conselho & 2 & 73,74 \\
\hline & Garanhuns & 2 & 72,73 \\
\hline \multirow[t]{2}{*}{8} & Belém do São Francisco & 3 & 73,74 \\
\hline & Petrolândıa & 2 & 72,74 \\
\hline \multirow[t]{2}{*}{9} & Tacaratu & 2 & 72,74 \\
\hline & .2 - Esquemas estatisti & & \\
\hline
\end{tabular}




\begin{tabular}{lc}
\hline Fontes de Variação & G.L. \\
\hline Nitrogénio $(N)$ & 2 \\
Fósforo $(P)$ & 2 \\
Potässıo $(K)$ & 2 \\
Interação (NxP) & 4 \\
Interação (NxK) & 4 \\
Interação (PxK) & 4 \\
Interação (NxPxK) (parte não confundida) & 6 \\
Blocos & 5 \\
Resíduo & 24 \\
\hline T o t a l & 53 \\
\hline
\end{tabular}

Para o calcário, o esquema usado é o que se segue:

\begin{tabular}{lc}
\hline Fontes de Variação & G.L. \\
\hline Calcário & 2 \\
Blocos & 5 \\
Resíduo & 10 \\
\hline Tota 1 & 17 \\
\hline
\end{tabular}

As análises conjuntas foram feitas de acordo com o modelo matemático:

$$
x_{i j k}=m+t_{i}+l_{k}+b_{j k}+(t \ell)_{i k}+e_{i j k} .
$$


28 .

com $1=1,2, \ldots, I$

$$
\begin{aligned}
& j=1,2, \ldots, j ; \\
& k=1,2, \ldots, k ;
\end{aligned}
$$

onde, $x_{i j k}$ corresponde à produçäo da parcela do tratamento $i$, no blo co $j$ e no ensaio $k$;

m representa a média geral do ensaio:

$t_{1}$ refere-se ao efeito do tratamento i;

$\ell_{k}$ representa o efeito do local $\underline{k}_{z}$

$b_{j k}$ representa o efeito do bloco $\underline{j}$ no local $k_{j}$

$(t \ell)_{1 k}$ é o efeito da interação do tratamento 1 com o local $\underline{k}$;

$e_{i j k}$ corresponde ao efelto do acaso.

De acordo com esse modelo, os esquemas das anälises conjuntas para o nitrogênı, o fósforo, o potássio e o calcário são respectivamente:

\begin{tabular}{lc}
\hline Funtes de Variação & G.L. \\
\hline Ensaios $(E)$ & $K-1$ \\
Nitrogênio $(N)$ & 2 \\
Interação $(E \times N)$ & $2(K-1)$ \\
Residuo médio & $24 K$ \\
\hline
\end{tabular}




\begin{tabular}{lc}
\hline Fontes de Variação & G.L. \\
\hline Ensaios $(E)$ & $K-1$ \\
Fósforo $(P)$ & 2 \\
Interação (ExP) & $2(K-1)$ \\
Residuo mëdio & $24 K$ \\
\hline
\end{tabular}

\begin{tabular}{lc}
\hline Fontes de Variação & G.L. \\
\hline Ensaios $(E)$ & $K-1$ \\
Potássio $(K)$ & 2 \\
Interação (ExK) & $2(K-1)$ \\
Residuo médto & $24 K$ \\
\hline
\end{tabular}

\begin{tabular}{lc}
\hline Fontes de Variação & G.L. \\
\hline Ensaios $(E)$ & $\mathrm{K}-1$ \\
Calcärio $(C)$ & 2 \\
Interação (ExC) & $2(\mathrm{~K}-1)$ \\
Residuo mëdio & $10 \mathrm{~K}$ \\
\hline
\end{tabular}

No caso em que os efeitos principais de $N, P, K$ e $C a$ apresentaram significāncia estatística e os graus de liberdade permitiram, fol feito o desdobramento desses em seus efeitos lineares e quadräticos. 
30 .

\subsection{3 - Estudo do modelo polinomial quadrātico}

\subsubsection{1 - Estimativas dos parâmetros e seus respec tivos intervalos de confiança}

Para a função de produção:

$$
\begin{aligned}
y= & a_{0}+a_{11} x_{1}^{2}+a_{22} x_{2}^{2}+a_{33} x_{3}^{2}+a_{12} x_{1} x_{2}+a_{13} x_{1} x_{3}+a_{23} x_{2} x_{3}+a_{14} x_{1}+ \\
& +a_{24} x_{2}+a_{34} x_{3} .
\end{aligned}
$$

onde, $x_{1}, x_{2}, x_{3}$ representam as doses de $N, P_{2} \mathrm{O}_{5}$ e $K_{2} \mathrm{O}_{\text {; }}$

$a_{11}, a_{22}, a_{33}$ são os coeficientes dos efeitos quadräticos, res pectivamente de $N, P$ e $K_{\text {: }}$

$a_{12}, a_{13}, a_{23}$ são os coeficientes das interações duplas:

$a_{14}, a_{24}, a_{34}$ são os coeficientes dos efeitos lineares respec tivamente de $N, P$ e $K$;

facilmente se obtém, através de métodos matriciais utilizados na teoría da regressão, as matrizes $X^{\prime} X=s e\left(X^{\prime} X\right)^{-1}=s^{-1}$, após a codificação das variáveis $x_{1}, x_{2}$ e $x_{3}$ em $-1,0$ e 1 , através da transfor mação:

$$
x_{i}=\frac{x_{1}-\bar{x}}{q} \text {, }
$$

onde: $x_{1}=0,50,100, q=50$;

$x_{2}=0,60,120, q=60$

$x_{3}=0,30,60, q=30$; 


$$
X=S=\left[\begin{array}{cccccccccc}
54 & 36 & 36 & 36 & 0 & 0 & 0 & 0 & 0 & 0 \\
36 & 36 & 24 & 24 & 0 & 0 & 0 & 0 & 0 & 0 \\
36 & 24 & 36 & 24 & 0 & 0 & 0 & 0 & 0 & 0 \\
36 & 24 & 24 & 36 & 0 & 0 & 0 & 0 & 0 & 0 \\
0 & 0 & 0 & 0 & 24 & 0 & 0 & 0 & 0 & 0 \\
0 & 0 & 0 & 0 & 0 & 24 & 0 & 0 & 0 & 0 \\
0 & 0 & 0 & 0 & 0 & 0 & 24 & 0 & 0 & 0 \\
0 & 0 & 0 & 0 & 0 & 0 & 0 & 36 & 0 & 0 \\
0 & 0 & 0 & 0 & 0 & 0 & 0 & 0 & 36 & 0 \\
0 & 0 & 0 & 0 & 0 & 0 & 0 & 0 & 0 & 36
\end{array}\right]
$$

$$
\left(X^{\prime} X\right)^{-1}=S^{-1}=\left[\begin{array}{cccccccccc}
7 / 54 & -1 / 18 & -1 / 18 & -1 / 18 & 0 & 0 & 0 & 0 & 0 & 0 \\
-1 / 18 & 1 / 12 & 0 & 0 & 0 & 0 & 0 & 0 & 0 & 0 \\
-1 / 18 & 0 & 1 / 12 & 0 & 0 & 0 & 0 & 0 & 0 & 0 \\
-1 / 18 & 0 & 0 & 1 / 12 & 0 & 0 & 0 & 0 & 0 & 0 \\
0 & 0 & 0 & 0 & 1 / 24 & 0 & 0 & 0 & 0 & 0 \\
0 & 0 & 0 & 0 & 0 & 1 / 24 & 0 & 0 & 0 & 0 \\
0 & 0 & 0 & 0 & 0 & 0 & 1 / 24 & 0 & 0 & 0 \\
0 & 0 & 0 & 0 & 0 & 0 & 0 & 1 / 36 & 0 & 0 \\
0 & 0 & 0 & 0 & 0 & 0 & 0 & 0 & 1 / 36 & 0 \\
0 & 0 & 0 & 0 & 0 & 0 & 0 & 0 & 0 & 1 / 36
\end{array}\right]
$$


A matriz $X ' Y$ é obtida pré-multiplicando-se a matriz $X$ pelo vetor das observaçöes $Y$. Verifica-se que:

$$
X^{\prime} Y=\left[\begin{array}{l}
G \\
N_{0}+N_{2} \\
P_{0}+P_{2} \\
K_{0}+K_{2} \\
N^{\prime} \times P^{\prime} \\
N^{\prime} \times K^{\prime} \\
P^{\prime} \times K^{\prime} \\
N^{\prime} \\
P^{\prime} \\
K^{\prime}
\end{array}\right]
$$

onde, $N_{0}, P_{0}$ e $K_{0}$ são os totais das parcelas sem nitrogênio, fósforo e potássio, respectivamente:

$N_{2}, P_{2}$ e $K_{2}$ são os totais das parcelas com a dose 2 de $N, P$ e K, respectivamente:

$N^{\prime} X^{\prime}, N^{\prime} \times K^{\prime}$ e $P^{\prime} \times K^{\prime}$ são os efeitos das interações lineares. isto é:

$$
\begin{aligned}
& N^{\prime} \times P^{\prime}=N_{0} P_{0}+N_{2} P_{2}-N_{0} P_{2}-N_{2} P_{0}, \\
& N^{\prime} \times K^{\prime}=N_{0} K_{0}+N_{2} K_{2}-N_{0} K_{2}-N_{2} K_{0}= \\
& P^{\prime} \times K^{\prime}=P_{0} K_{0}+P_{2} K_{2}-P_{0} K_{2}-P_{2} K_{0}=
\end{aligned}
$$


$N^{\prime}, P^{\prime} e K^{\prime}$ são os efeitos lineares de nitrogênio, fósforo e potássio, respectivamente, ou seja:

$$
\begin{aligned}
& N^{\prime}=N_{2}-N_{0}, \\
& P^{\prime}=P_{2}-P_{0}, \\
& K^{\prime}=K_{2}-K_{0} .
\end{aligned}
$$

0 vetor das estimativas dos parâmetros $\dot{B}$ é obtido co mo se segue:

$$
\hat{B}=S^{-1} X \cdot Y=\left[\begin{array}{l}
a_{0} \\
a_{12} \\
a_{22} \\
a_{33} \\
a_{12} \\
\hat{a}_{13} \\
a_{23} \\
\hat{a}_{14} \\
a_{24} \\
a_{34}
\end{array}\right]
$$

onde: $\hat{a}_{0}=\frac{7}{54} G-\frac{1}{18}\left[\left(N_{0}+N_{2}\right)+\left(P_{0}+P_{2}\right)+\left(K_{0}+K_{2}\right)\right]$

$$
\begin{aligned}
& =\frac{7}{54} G-\frac{1}{18}\left[\left(G-N_{1}\right)+\left(G-P_{1}\right)+\left(G-K_{1}\right)\right] \\
& =\frac{1}{18}\left(N_{1}+P_{1}+K_{1}\right)-\frac{G}{27}
\end{aligned}
$$




$$
\begin{aligned}
& \hat{a}_{12}=\frac{N_{0}+N_{2}}{12}-\frac{G}{18}=\frac{N_{0}-2 N_{1}+N_{2}}{36}=\frac{N^{\prime \prime}}{36} ; \\
& \hat{a}_{22}=\frac{P_{0}+P_{2}}{12}-\frac{G}{18}=\frac{P_{0}-2 P_{1}+P_{2}}{36}=\frac{P^{\prime \prime}}{36} ; \\
& \hat{a}_{33}=\frac{K_{0}+K_{2}}{12}-\frac{G}{18}=\frac{K_{0}-2 K_{1}+K_{2}}{36}=\frac{K^{\prime \prime}}{36}: \\
& \hat{a}_{12}=\frac{N^{\prime} \times P^{\prime}}{24} ; \\
& \hat{a}_{13}=\frac{N^{\prime} \times K^{\prime}}{24} ; \\
& \hat{a}_{23}=\frac{P^{\prime} \times K^{\prime}}{24} \\
& \hat{a}_{14}=\frac{P^{\prime}}{36}: \\
& 36
\end{aligned}
$$

onde. $N "$ " $P "$ e $K "$, säo os efeltos quadräticos, respectivamente de $N_{*}$ $P$ e K.

No caso dos grupos de ensaios, o vetor $\vec{B}$ serla:

$$
\beta=s^{-1} x \cdot \bar{P}
$$

onde $\bar{Y} \ddot{e}$ o vetor das médias das observações. 
A matriz de dispersão $\underline{D}$ das variâncias e covariàncias dos paràmetros seria, para o caso dos ensalos individuais:

$$
D=S^{-1} \sigma^{2} \quad \text {. }
$$

portanto,

$$
\begin{aligned}
v\left(\hat{a}_{0}\right) & =\frac{7}{54} \sigma^{2}, \\
V\left(\hat{a}_{13}\right) & =V\left(\hat{a}_{22}\right)=V\left(\hat{a}_{33}\right)=\frac{1}{12} \sigma^{2}, \\
V\left(\hat{a}_{12}\right) & =V\left(\hat{a}_{13}\right)=V\left(\hat{a}_{23}\right)=\frac{1}{24} \sigma^{2}, \\
V\left(\hat{a}_{14}\right) & =V\left(\hat{a}_{24}\right)=V\left(\hat{a}_{34}\right)=\frac{1}{36} \sigma^{2}, \\
\operatorname{cov}\left(\hat{a}_{0}, \hat{a}_{11}\right) & =\operatorname{cov}\left(\hat{a}_{0}, \hat{a}_{22}\right)=\operatorname{cov}\left(\hat{a}_{0}, \hat{a}_{33}\right)=-\frac{1}{18} \sigma^{2} .
\end{aligned}
$$

As demais covarianncias são todas nulas.

As estimativas dessas varianncias e covariàncias são obtidas substituindo-se $\sigma^{2}$ pela estimativa da variància residual $s^{2}=$ QMR。

Para os grupos de $N$ ensaios, a matriz de dispersão será:

$$
D=\frac{S^{-1}}{N} \sigma^{2} \text {. }
$$

Portanto, as variâncias e covariâncias dos parâmetros nesse caso serão as mesmas dos ensalos individuals, divididas por $\underline{N}$ e suas estimativas são obtidas substituindo-se $\sigma^{2}$ por $s^{2}$, no caso a 
36 .

estimativa da variância residual média de cada grupo.

Com as estimativas das variàncias das estimativas dos paràmetros, facilmente chega-se aos seus intervalos de confiança, ad mitindo-se que tenham uma distribuição aproximadamente normal:

$$
\begin{aligned}
& I C=\left[\hat{a}_{0}-t s \sqrt{\frac{7}{54}}, \hat{a}_{0}+t s \sqrt{\frac{7}{54}}\right], \\
& I C=\left[a_{i 1}-t \frac{s}{\sqrt{12}}, \hat{a}_{i 1}+t \frac{s}{\sqrt{12}}\right] \quad(i=1,2,3), \\
& I C=\left[\hat{a}_{i j}-t \frac{s}{\sqrt{24}}, \hat{a}_{i j}+t \frac{s}{\sqrt{24}}\right] \quad(1=1,2 ; j=2,3 ; i \neq j), \\
& I C=\left[\hat{a}_{i 4}-t \frac{s}{6}, \hat{a}_{i 4}+t \frac{s}{6}\right] \quad(i=1,2,3),
\end{aligned}
$$

Ao nivel de $95 \%$ de probabilidade e tomando-se $t=2$,

virá:

$$
\begin{aligned}
& I C=\left[a_{0}-0,72 \mathrm{~s}, \hat{a}_{0}+0,72 \mathrm{~s}\right], \\
& I C=\left[\hat{a}_{i 1}-0,58 \mathrm{~s}, \hat{a}_{1 j}+0,58 \mathrm{~s}\right], \\
& I C=\left[\hat{a}_{1 j}-0,41 \mathrm{~s}, \hat{a}_{1 j}+0,41 \mathrm{~s}\right], \\
& I C=\left[\hat{a}_{14}-0,33 \mathrm{~s}, \hat{a}_{14}+0,33 \mathrm{~s}\right],
\end{aligned}
$$


Consequentemente, para os grupos de $\mathrm{N}$ ensaios tere-

mos:

$$
\begin{aligned}
& I C=\left[\hat{a}_{0}-0,72 \frac{s}{\sqrt{N}}, \hat{a}_{0}+0,72 \frac{s}{\sqrt{N}}\right], \\
& I C=\left[\hat{a}_{11}-0,58 \frac{s}{\sqrt{N}}, \hat{a}_{11}+0,58 \frac{s}{\sqrt{N}}\right], \\
& I C=\left[\hat{a}_{i j}-0,41 \frac{s}{\sqrt{N}}, a_{i j}+0,41 \frac{s}{\sqrt{N}}\right], \\
& I C=\left[a_{14}-0,33 \frac{s}{\sqrt{N}}, a_{i 4}+0,33 \frac{s}{\sqrt{N}}\right],
\end{aligned}
$$

Para os grupos de ensaios, foi aplicado o teste $t$ pa ra os coeficientes das equações de regressão através da fórmula:

$$
t=\frac{\hat{a}-0}{\sqrt{b(a ̂)}},
$$

onde à é a estimativa do parâmetro em questão.

$$
\text { i. }
$$

\subsubsection{2 - Intervalos de confiança das estimativas dos rendimentos}

CAMPOS (1967) por inspeção do quadro $I$, onde determi nou as funções estimadoras dos 27 tratamentos estudados nos ensaios fatoriais, chegou à conclusão de que as expressões das variâncias das estimativas dos rendimentos, formavam apenas 4 grupos distintos, a saber: 


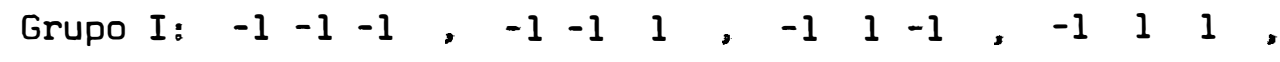

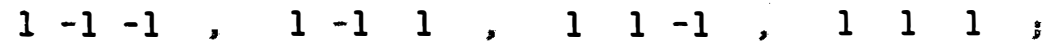

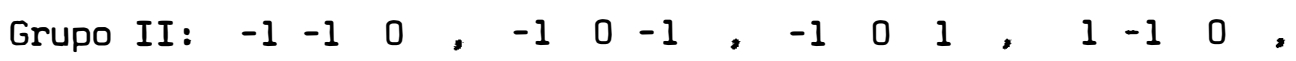

$$
\begin{aligned}
& \begin{array}{llllllllllllll} 
& -1 & 1 & 0 & 1 & -1 & 0 & 1 & 1 & 0 & -1 & -1
\end{array} \text {. }
\end{aligned}
$$

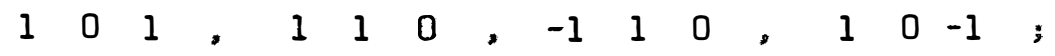

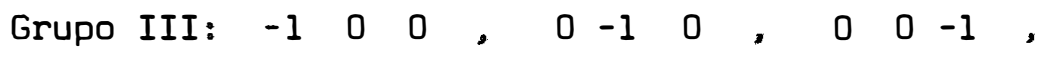

$$
100,0010,0001 \text {, }
$$

Grupo IV: $0 \quad 0 \quad 0$.

Partindo-se da estimativa $\hat{y}$ e aplicando-se as propriedades da variância nessa função vamos ter:

$$
\begin{aligned}
\hat{y}= & \hat{a}_{0}+\hat{a}_{11} x_{1}^{2}+\hat{a}_{22} x_{2}^{2}+\hat{a}_{33} x_{3}^{2}+\hat{a}_{12} x_{1} x_{2}+\hat{a}_{13} x_{1} x_{3}+\hat{a}_{23} x_{2} x_{3}+\hat{a}_{14} x_{1}+ \\
& +\hat{a}_{24} x_{2}+\hat{a}_{34} x_{3}, \\
V(\hat{y})=V\left(\hat{a}_{0}\right) & +x_{1}^{4} V\left(\hat{a}_{11}\right)+x_{2}^{4} V\left(\hat{a}_{22}\right)+x_{3}^{4} V\left(\hat{a}_{33}\right)+x_{1}^{2} x_{2}^{2} V\left(\hat{a}_{12}\right)+x_{1}^{2} x_{3}^{2} V\left(\hat{a}_{13}\right)+ \\
& +x_{2}^{2} x_{3}^{2} V\left(\hat{a}_{23}\right)+x_{1}^{2} V\left(\hat{a}_{14}\right)+x_{2}^{2} V\left(\hat{a}_{24}\right)+x_{3}^{2} V\left(\hat{a}_{34}\right)+2 x_{1}^{2} \operatorname{CoV}\left(\hat{a}_{0}, \hat{a}_{13}\right)+ \\
& +2 x_{2}^{2} \operatorname{coV}\left(\hat{a}_{0}, \hat{a}_{22}\right)+2 x_{3}^{2} \operatorname{coV}\left(\hat{a}_{0}, \hat{a}_{33}\right)
\end{aligned}
$$

então, para os quatro grupos, as expressões das estimativas das variàncias dos rendimentos e seus respectivos intervalos de confiança ao nível de $95 \%$ de probabilidade dos grupos de ensaios são:

Grupo I:

$$
\nabla(\bar{\gamma})=\nabla\left(\hat{a}_{0}\right)+3 V\left(\hat{a}_{11}\right)+3 V\left(\hat{a}_{1 j}\right)+3 V\left(\hat{a}_{i_{4}}\right)+6 \operatorname{CoV}\left(\hat{a}_{0}, \hat{a}_{1 i}\right)
$$




$$
\begin{aligned}
& \hat{V}(\hat{y})=\frac{55}{216} \cdot \frac{s^{2}}{N}, \\
& I C=\left[\hat{y}-1,01 \frac{s}{\sqrt{N}}, \hat{y}+1,01 \frac{s}{\sqrt{N}}\right] .
\end{aligned}
$$

Grupo II:

$$
\begin{aligned}
& \hat{V}(\hat{y})=V\left(\hat{a}_{0}\right)+2 V\left(\hat{a}_{11}\right)+V\left(\hat{a}_{1 j}\right)+2 V\left(\hat{a}_{14}\right)+4 \operatorname{Cô}\left(\hat{a}_{0}, \hat{a}_{1 i}\right) \\
& =\frac{37}{216} \cdot \frac{s^{2}}{N} \text {, } \\
& I C=\left[\bar{y}-0,83 \frac{s}{\sqrt{N}}, \bar{y}+0,83 \frac{s}{\sqrt{N}}\right] .
\end{aligned}
$$

Grupo III:

$$
\begin{aligned}
& \nabla(\hat{y})= \nabla\left(\hat{a}_{0}\right)+\nabla\left(\hat{a}_{i 1}\right)+\nabla\left(\hat{a}_{i 4}\right)+2 \operatorname{Cov}\left(\hat{a}_{0}, \hat{a}_{i i}\right) \\
&= \frac{7}{54} \cdot \frac{s^{2}}{N}, \\
& I C=\left[\bar{y}-0,72 \frac{s}{\sqrt{N}}, \hat{y}+0,72 \frac{s}{\sqrt{N}}\right] .
\end{aligned}
$$

Grupo IV:

$$
\begin{aligned}
V(\hat{y})=V\left(\hat{a}_{0}\right)= & \frac{7}{54} \cdot \frac{s^{2}}{N} . \\
I C & =\left[\hat{y}-0,72 \frac{s}{\sqrt{N}}, \hat{y}+0,72 \frac{s}{\sqrt{N}}\right] .
\end{aligned}
$$


40.

4.2.3.3 - Doses ótimas dos fatores $x_{1}, x_{2} e x_{3}$ ob tidas atravēs da superfície de resposta

Partindo da função de receita 1 lquida:

$$
L\left(x_{1}, x_{2}, x_{3}\right)=w \bar{y}-t_{1} x_{1}-t_{2} x_{2}-t_{3} x_{3}-m \text {, }
$$

onde: $\hat{y}=\hat{a}_{0}+\hat{a}_{11} x_{1}^{2}+\hat{a}_{22} x_{2}^{2}+\hat{a}_{33} x_{3}^{2}+\hat{a}_{12} x_{1} x_{2}+\hat{a}_{13} x_{1} x_{3}+\hat{a}_{23} x_{2} x_{3}+\hat{a}_{14} x_{1}+$

$$
+\hat{a}_{24} x_{2}+\hat{a}_{34} x_{3}
$$

$w=$ preço de venda do produto:

$t_{1}=$ preço da dose unitäria $x_{1}$ :

$t_{2}=$ preço da dose unitäria $x_{2}$;

$t_{3}=$ preço da dose unitária $x_{3}$ :

$m=$ são as despesas fixas.

As condições necessárias para que a função de receita liquida tenha um máximo em $\left(x_{1}^{+}, x_{2}^{+}, x_{3}^{+}\right)$, são as seguintes:

$$
\begin{aligned}
& d L\left(x_{1}, x_{2}, x_{3}\right) \equiv 0, \quad d^{2} L\left(x_{1}, x_{2}, x_{3}\right)<0 . \\
& \text { Da primeira condição, derivando-se parcialmente a fun }
\end{aligned}
$$
çäo de receita Ilquida en relação às variáveis $x_{1}, x_{2} e x_{3}$, chega-se ao seguinte sistema de equações:

$$
\left\{\begin{array}{l}
2 \hat{a}_{11} x_{1}+\hat{a}_{12} x_{2}+\hat{a}_{13} x_{3}=\frac{t_{1}}{w}-\hat{a}_{14} \\
\hat{a}_{12} x_{1}+2 \hat{a}_{22} x_{2}+\hat{a}_{23} x_{3}=\frac{t_{2}}{w}-\hat{a}_{24} \\
\hat{a}_{13} x_{1}+\hat{a}_{23} x_{2}+2 \hat{a}_{33} x_{3}=\frac{t}{w}-\hat{a}_{34}
\end{array},\right.
$$


A solução do sistema de equações apresentado pode nos conduzir a uma receita líquida máxima se a segunda condiçäo for verificada, ou seja, se a matriz $M$ das derivadas parciais de segunda ordem for definida negativa. Assim, segundo PERLIS (1958), temos:

$$
M=w\left[\begin{array}{ccc}
2 \hat{a}_{11} & \hat{a}_{12} & \hat{a}_{13} \\
\hat{a}_{12} & 2 \hat{a}_{22} & \hat{a}_{23} \\
\hat{a}_{13} & \hat{a}_{23} & 2 \hat{a}_{33}
\end{array}\right]=w A \text {. }
$$

Como w é um número real positivo, se a matriz $A$ for definida negativa, assegura-se então a existência do máximo. Logo, através de operações de congruēncla com a matriz $A$, e segundo RAO (1952), obtém-se a matriz diagonal D.

$$
D=\left[\begin{array}{ccc}
\Delta_{1} & 0 & 0 \\
0 & \Delta_{2} / \Delta_{1} & 0 \\
0 & 0 & \Delta_{3} / \Delta_{2}
\end{array}\right],
$$

onde: $\Delta_{1}=2 \hat{a}_{11}$;

$$
\begin{aligned}
& \Delta_{2}=\left|\begin{array}{ll}
2 \hat{a}_{11} & \hat{a}_{12} \\
\hat{a}_{12} & 2 \hat{a}_{22}
\end{array}\right| ; \\
& \Delta_{3}=|A|:
\end{aligned}
$$

então, se $\Delta_{1}<0_{3} \Delta_{2} / \Delta_{1}<0 ; \Delta_{3} / \Delta_{2}<0$; realmente a solução do sistema de equaçōes nos conduz a uma receita líquida máxima.

Com base nessa teoria, foram resolvidos os sistemas de equaçöes e discutido a existência do mäximo para os grupos de en saios, considerando-se para tal, as seguintes relações de preços w/t, 
42 .

obtidas na praça de Recife:

$$
\begin{aligned}
& \frac{w}{t_{1}}=0,1304, \\
& \frac{w}{t_{2}}=0,1551 . \\
& \frac{w}{t_{3}}=0,3866 .
\end{aligned}
$$

\subsubsection{4 - Cortes da superficie}

Os cortes da superficie são obtidos fixando-se duas das três variáveis em suas doses médias, no caso presente, $x_{i}=0$. Assim, para se estudar o comportamento da superfície quando são fixados fósforo e potássio, em suas doses médias, temos:

18 Corte: $x_{2}=0$ e $x_{3}=0$ :

$$
\hat{y}_{1}=\hat{y}\left(x_{1}, 0,0\right)=\hat{a}_{0}+\hat{a}_{14} x_{1}+\hat{a}_{11} x_{1}^{2} .
$$

Nesse caso, a função de receita líquida é:

$$
L_{1}=L\left(x_{1}, 0,0\right)=w \bar{y}_{1}-t_{1} x_{1}-m,
$$

e as condições para se obter o máximo são:

$$
\frac{d L_{1}}{d x_{1}}=0 \quad, \quad \frac{d^{2} L_{1}}{d x_{1}^{2}}<0 .
$$

Da primeira condição facilmente se obtém: 


$$
x_{1}^{*}=\frac{1}{2 \bar{a}_{11}}\left(\frac{t_{1}}{w}-\hat{a}_{14}\right)
$$

Por outro lado,

$$
\frac{d^{2} L_{1}}{d x_{1}^{2}}=2 w \hat{a}_{11}
$$

Como $w>0$, a condição para que a função de receita Ifquida seja mäxima em $x_{1}^{*}$, nesse caso, é que tenhamos $\hat{a}_{11}<0$.

Segundo PIMENTEL GOMES (1953), uma aproximação da va riância de $x_{1}^{*}$ pode ser obtida através da fórmula de Taylor. Esse processo, de acordo com D'AULISIO (1976), é recomendável, embora dê intervalos de confiança um tanto curtos. Assim, por diferenciação, vem:

$$
d x_{1}^{*}=\frac{1}{2 \hat{a}_{11}} d \hat{a}_{14}-\frac{1}{2 \hat{a}_{11}^{2}}\left(\frac{t_{1}}{w}-\hat{a}_{14}\right) d \hat{a}_{11} \text {. }
$$

então,

$$
\begin{aligned}
V\left(x_{1}^{*}\right)=\frac{1}{4 \hat{a}_{11}^{2}} V\left(\hat{a}_{14}\right) & +\frac{1}{4 \hat{a}_{11}^{-4}}\left(\frac{t_{1}}{w}-\hat{a}_{14}\right)^{2} \nabla\left(\hat{a}_{11}\right)+ \\
& +\frac{1}{2 \hat{a}_{11}^{3}}\left(\frac{t}{w}-\hat{a}_{14}\right) \operatorname{Côv}\left(\hat{a}_{11}, \hat{a}_{14}\right),
\end{aligned}
$$

e como $\operatorname{Cov}\left(\hat{a}_{11}, \hat{a}_{14}\right)=0$, e dispondo-se de $V\left(\hat{a}_{14}\right)$ e $V\left(\hat{a}_{11}\right)$, chega-se facilmente à expressão da estimativa da variância de $x_{1}^{*}$ para os gru pos de ensaios calculados neste trabal ho: 


$$
\left.\nabla\left(x_{1}^{*}\right)=\frac{s^{2}}{48 N \hat{a}_{11}^{2}}\left[\frac{\frac{t_{1}}{w}-\hat{a}_{14}}{\left(\frac{\hat{a}_{11}}{2}\right.}\right)^{2}+\frac{1}{3}\right],
$$

e admitindo-se que a distribuição de $x_{1}^{*}$ seja aproximadamente normal, - intervalo de confiança de $x_{1}^{*}$ é:

$$
I C=\left[x_{1}^{*}-\operatorname{ts}\left(x_{1}^{*}\right), x_{1}^{*}+t s\left(x_{1}^{*}\right)\right]
$$

Fizeram-se analogamente os outros cortes.

$$
\begin{aligned}
& 28 \text { Corte: } x_{1}=0, x_{3}=0 \\
& \hat{y}_{2}=\hat{y}\left(0, x_{2}, 0\right)=\hat{a}_{0}+\hat{a}_{24} x_{2}+\hat{a}_{22} x_{2}^{2} \\
& L_{2}=L\left(0, x_{2}, 0\right)=w \bar{y}_{2}-t_{2} x_{2}-m \\
& \frac{d L_{2}}{d x_{2}}=0 \Longrightarrow x_{2}^{*}=\frac{1}{2 \tilde{a}_{22}}\left(\frac{t_{2}}{w}-\tilde{a}_{24}\right) \\
& \frac{d^{2} L_{2}}{d x_{2}^{2}}<0 \Rightarrow \hat{a}_{22}<0 \\
& \hat{V}\left(x_{2}^{*}\right)=\frac{s^{2}}{48 N \hat{a}_{22}^{2}}\left[\frac{\left.\frac{t_{2}}{w}-\hat{a}_{24}\right)^{2}+\frac{1}{3}}{\hat{a}_{22}}\right] \\
& I C=\left[x_{2}^{*}-\operatorname{ts}\left(x_{2}^{*}\right), x_{2}^{*}+t s\left(x_{2}^{*}\right)\right]
\end{aligned}
$$


38 Corte: $x_{1}=0, x_{2}=0$

$$
\begin{aligned}
& \bar{y}_{3}=\hat{y}\left(0,0, x_{3}\right)=\hat{a}_{0}+\vec{a}_{34} x_{3}+\hat{a}_{3} x_{3}^{2}, \\
& L_{3}=L\left(0,0, x_{3}\right)=w \hat{y}_{3}-t_{3} x_{3}-m, \\
& \frac{d L_{3}}{d x_{3}}=0 \Longrightarrow x_{3}^{*}=\frac{1}{2 \hat{a}_{33}}\left(\frac{t_{3}}{w}-\hat{a}_{34}\right), \\
& \frac{d^{2} L_{3}}{d x_{3}^{2}}<0 \Rightarrow \hat{a}_{33}<0, \\
& V\left(x_{3}^{*}\right)=\frac{1}{48 N a_{33}^{2}}\left[\frac{\left.\frac{t_{3}}{w}-a_{34}\right)^{2}+\frac{1}{3}}{a_{33}}\right] s^{2} \text {, } \\
& I C=\left[x_{3}^{*}-\operatorname{ts}\left(x_{3}^{*}\right), x_{3}^{*}+t s\left(x_{3}^{*}\right)\right] \text {. }
\end{aligned}
$$

4.2.3.5 - Anälise da regressão e coeficiente de determinação total da superfície de resposta

De NEVES et alii (1960) e MORAES (1969), podemos con cluir que o esquema da análise de variância da regressão é o seguin te. 


\begin{tabular}{lc}
\hline Fontes de Variação & G.L. \\
\hline Regressão & 9 \\
Desvios da Regressão & 15 \\
- & \\
\hline Tratamentos & 24 \\
Blocos & 5 \\
Residuo & 24 \\
\hline To t a l & 53 \\
\hline
\end{tabular}

MORAES (1969) determinou a expressão da soma de quadrados da regressão para o caso do fatorial de $3^{3}$ com uma repetição. Para o caso de duas repetições, obtêm-se de maneira anäloga, como se segue:

$$
\text { SQ Reg }=\hat{B} \cdot X \cdot Y-C \text {. }
$$

sendo,

$$
\begin{aligned}
\hat{B}^{\prime} X^{\prime} Y=\left[\frac{1}{18}\left(N_{1}+P_{1}+K_{1}\right)-\frac{G}{27}\right] G & +\frac{N^{\prime \prime}}{36}\left(N_{0}+N_{2}\right)+\frac{P^{\prime \prime}}{36}\left(P_{0}+P_{2}\right)+ \\
& +\frac{K^{\prime \prime}}{36}\left(K_{0}+K_{2}\right)+\frac{\left(N^{\prime} \times P^{\prime}\right)^{2}}{24}+ \\
& +\frac{\left(N^{\prime} \times K^{\prime}\right)^{2}}{24}+\frac{\left(P^{\prime} \times K^{\prime}\right)^{2}}{24}+ \\
& +\frac{N^{2}}{36}+\frac{P^{2}}{36}+\frac{K^{2}}{36} .
\end{aligned}
$$


Desenvolvendo a $2^{a}$ expressão do $2^{\circ}$ membro vem:

$$
\begin{aligned}
\frac{N^{\circ 0}}{36}\left(N_{0}+N_{2}\right) & =\frac{N^{\prime \prime}}{108}\left(3 N_{0}+3 N_{2}\right)=\frac{N^{\prime \prime}}{108}\left(3 N_{0}+3 N_{2}+2 N_{1}-2 N_{1}\right) \\
& =\frac{N^{\prime \prime}}{108}\left[\left(N_{0}-2 N_{1}+N_{2}\right)+2\left(N_{0}+N_{1}+N_{2}\right)\right] \\
& =\frac{N^{\prime \prime}}{108}\left(N^{\prime \prime}+2 G\right)=\frac{N^{\prime \prime}}{108}+\frac{N^{\prime \prime} G}{54} .
\end{aligned}
$$

analogamente,

$$
\frac{P^{\prime \prime}}{36}\left(P_{0}+P_{2}\right)=-\frac{P^{2}}{108}+\frac{P^{\prime \prime} G}{54}
$$

e,

$$
\frac{K^{\prime \prime}}{36}\left(K_{0}+K_{2}\right)=\frac{K^{\frac{2}{1}}}{108}+\frac{K^{\prime \prime} G}{54},
$$

então:

$$
\begin{aligned}
\hat{B} \cdot X ' Y=\frac{G}{18}\left(N_{1}+P_{1}+K_{1}\right) & -\frac{G^{2}}{27}+\frac{N^{\prime \prime} G}{54}+\frac{P^{\prime \prime} G}{54}+\frac{K^{\prime \prime} G}{54}+\frac{N^{2}}{108}+\frac{P^{2}}{108}+ \\
& +\frac{K^{\prime \prime}}{108}+\frac{\left(N^{\prime} \times P^{\prime}\right)^{2}}{24}+\frac{\left(N^{\prime} \times K^{\prime}\right)^{2}}{24}+\frac{\left(P^{\prime} \times K^{\prime}\right)^{2}}{24}+ \\
& +\frac{N^{2}}{36}+\frac{P^{2}}{36}+\frac{K^{2}}{36}
\end{aligned}
$$

Como:

$$
\frac{N^{2}}{108}=\frac{\left(N_{0}-2 N_{1}+N_{2}\right)^{2}}{108}=\text { SQN", }
$$


48.

$$
\begin{aligned}
& \frac{P^{2}}{108}=\frac{\left(P_{0}-2 P_{1}+P_{2}\right)^{2}}{108}=\operatorname{sopn} \\
& \frac{K^{2}}{108}=\frac{\left(K_{0}-2 K_{1}+K_{2}\right)^{2}}{108}=\operatorname{SOK}^{\prime \prime} \text {, } \\
& \frac{N^{2}}{36}=\frac{\left(N_{2}-N_{0}\right)^{2}}{36}=\text { SQN } \\
& \frac{P^{2}}{36}=\frac{\left(P_{2}-P_{0}\right)^{2}}{36}=S Q P \\
& \frac{K^{2}}{36}=\frac{\left(K_{2}-K_{0}\right)^{2}}{36}=S Q K \\
& \frac{\left(N^{\prime} \times P^{\prime}\right)^{2}}{24}=\frac{\left(N_{0} P_{0}+N_{2} P_{2}-N_{0} P_{2}-N_{2} P_{0}\right)^{2}}{24}=S Q^{\prime} \times P^{\prime}, \\
& \frac{\left(N^{\prime} \times K^{\prime}\right)^{2}}{24}=\frac{\left(N_{0} K_{0}+N_{2} K_{2}-N_{0} K_{2}-N_{2} K_{0}\right)}{24}=S Q N^{\prime} \times K^{\prime} . \\
& \frac{\left(P^{\prime} \times K^{\prime}\right)^{2}}{24}=\frac{\left(P_{0} K_{0}+P_{2} K_{2}-P_{0} K_{2}-P_{2} K_{0}\right)^{2}}{24}=S P^{\prime} \times K^{\prime},
\end{aligned}
$$

então:

$$
\begin{aligned}
\hat{B}^{\prime} X^{\prime} Y=\frac{G}{18}\left(N_{1}+P_{1}+K_{1}\right) & -\frac{G^{2}}{27}+\frac{N^{\prime \prime} G}{54}+\frac{P^{\prime \prime} G}{54}+\frac{K^{\prime \prime} G}{54}+S Q N^{\prime \prime}+ \\
& +S Q P^{\prime \prime}+S Q K^{\prime \prime}+S Q N^{\prime}+S Q P^{\prime}+ \\
& +S^{\prime}+K^{\prime}+\text { SQN' }^{\prime} \times P^{\prime}+S^{\prime} N^{\prime} \times K^{\prime}+ \\
& +S^{\prime} \times P^{\prime} \times K^{\prime} .
\end{aligned}
$$


Desenvolvendo os cinco primeiros termos do $2^{8}$ membro da expressão anterior vem:

$$
\begin{aligned}
& \frac{3 G}{54}\left(N_{1}+P_{1}+K_{1}\right)-\frac{G^{2}}{27}+\frac{N^{\prime \prime} G}{54}+\frac{P " G}{54}+\frac{K^{\prime \prime} G}{54}= \\
= & \frac{G}{54}\left(3 N_{1}+3 P_{1}+3 K_{1}+N_{0}-2 N_{1}+N_{2}+P_{0}-2 P_{1}+P_{2}+K_{0}-2 K_{1}+K_{2}\right)-\frac{G^{2}}{27}, \\
= & \frac{G}{54}\left[\left(N_{0}+N_{1}+N_{2}\right)+\left(P_{0}+P_{1}+P_{2}\right)+\left(K_{0}+K_{1}+K_{2}\right)\right]-\frac{G^{2}}{27}, \\
= & \frac{3 G^{2}}{54}-\frac{G^{2}}{27}=\frac{G^{2}}{54}=C .
\end{aligned}
$$

logo:

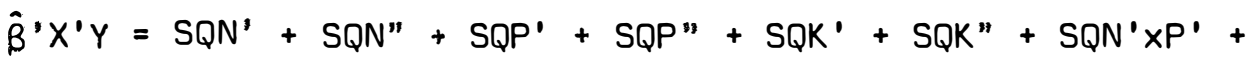

$$
\begin{aligned}
& +S Q N^{\prime} \times K^{\prime}+\text { SQP' } \times K^{\prime}+C^{\prime}
\end{aligned}
$$

então:

$$
\begin{aligned}
& \text { SQ Reg = } \hat{B}^{\prime} X^{\prime} Y-C=\mathrm{SQN}^{\prime}+\mathrm{SQN}^{\prime \prime}+\mathrm{SQP}^{\prime}+\mathrm{SQP} \mathrm{P}^{\prime}+\mathrm{SQK} \mathrm{K}^{\prime}+\mathrm{SQK} \mathrm{K}^{\prime}+ \\
& +S Q N^{\prime} X P^{\prime}+S Q N^{\prime} X K^{\prime}+S Q P^{\prime} X K^{\prime},
\end{aligned}
$$

ou, simplesmente:

$S Q R e g=S Q N+S Q P+S Q K+S Q N^{\prime} \times P^{\prime}+S Q N^{\prime} \times K^{\prime}+S Q P^{\prime} \times K^{\prime}$.

Com esse resultado, os testes $F$ para regressão e para os desvios da regressão podem ser obtidos facilmente através de:

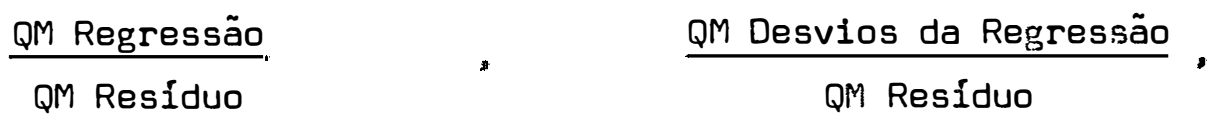


onde: SQ Desvios da Regressão = SQ Tratamentos - SQ Regressão.

0 coeficiente de determinação total $R^{2}$ pode ser calculado através do coeficiente de correlação $\underline{R}$ entre $y_{1}$ e $\hat{y}_{1}$ por intermédio da seguinte expressão:

$$
R=\frac{\sum y_{i} \hat{y}_{1}-\frac{\left(\Sigma y_{i}\right)\left(\Sigma \hat{y}_{i}\right)}{N}}{\sqrt{\left[y_{i}^{2}-\frac{\left(\Sigma y_{i}\right)^{2}}{N}\right]\left[\hat{y}_{i}^{2}-\frac{\left(\Sigma \hat{y}_{i}\right)^{2}}{N}\right]}} \cdot
$$

se reduz a:

$$
R=\sqrt{\frac{S Q \text { Regressão }}{S Q \text { Total }}}
$$

No caso de haver repetições para os tratamentos $e$. consequentemente, não serem necessariamente nulos, os desvios da regresão, a fórmula acima passa a ser:

$$
R=\sqrt{\frac{S Q \text { Regressão }}{S Q \text { Tratamentos }}}
$$

ou,

$$
R^{2}=\frac{S Q \text { Regressão }}{S Q \text { Tratamentos }}
$$

com a qual foram obtidos os coeficientes de deterninaçäo total. 
51.

\subsection{4 - 0 trinômio do segundo grau}

\subsubsection{1 - Estimativa dos parâmetros e de seus res - pectivos intervalos de confiança}

Dada a função de produção:

$$
y=a+b x+c x^{2}
$$

onde a variável $x_{1}$ foi codiflcada em $-1,0,1$, através da transformação:

$$
x_{i}=\frac{x_{i}-\bar{x}}{q} \text {. }
$$

matricialmente temos:

$$
X=\left[\begin{array}{ccc}
1 & -1 & 1 \\
1 & 0 & 0 \\
1 & 1 & 1
\end{array}\right], \quad Y=\left[\begin{array}{c}
\bar{Y}_{0} \\
\bar{Y}_{1} \\
\bar{Y}_{2}
\end{array}\right] \quad \text { e, } \quad X \cdot Y=\left[\begin{array}{ll}
\bar{Y}_{0}+\bar{Y}_{1}+\bar{Y}_{2} \\
\bar{Y}_{2}-\bar{Y}_{0} \\
\bar{Y}_{2}+\bar{Y}_{0}
\end{array}\right]
$$

onde $\bar{Y}_{0}, \bar{Y}_{1}$ e $\bar{Y}_{2}$ são respectivamente as produções médias relativas às doses 0,1 e 2 do nutriente em questão.

Assim, pela teoria da regressão:

$$
X \cdot X=S=\left[\begin{array}{lll}
3 & 0 & 2 \\
0 & 2 & 0 \\
2 & 0 & 2
\end{array}\right] \text {, }
$$




$$
\begin{aligned}
& (X \cdot X)^{-1}=s^{-1}=\left[\begin{array}{ccc}
1 & 0 & -1 \\
0 & 1 / 2 & 0 \\
-1 & 0 & 3 / 2
\end{array}\right], \\
& \hat{B}=s^{-1} X^{\prime} Y=\left[\begin{array}{c}
\bar{Y}_{1} \\
\left(\bar{Y}_{2}-\bar{Y}_{0}\right) / 2 \\
\left(\bar{Y}_{0}-2 \bar{Y}_{1}+\bar{Y}_{2}\right) / 2
\end{array}\right]
\end{aligned}
$$

Comparando-se esse resultado com o obtido nos cortes da superficie, facilmente chega-se às seguintes conclusões:

$$
\begin{aligned}
\text { Para o nitrogénio: } & \hat{G}=\hat{a}_{14} \\
\bar{c} & =\hat{a}_{11} \\
\text { Para o fósforo: } & \bar{c}=\hat{a}_{24} \\
\hat{c} & =\hat{a}_{22} \\
\text { Para o potässio: } & \bar{G}=\hat{a}_{34} \\
\bar{c} & =\hat{a}_{33}
\end{aligned}
$$

A matriz de dispersão, nesse caso, seria:

$$
D=\left[\begin{array}{ccc}
1 & 0 & -1 \\
0 & 1 / 2 & 0 \\
-1 & 0 & 3 / 2
\end{array}\right] \sigma^{2}
$$


ou, como no caso dos ensaios individuais, os totais de cada tratamen to são provenientes de 18 parcelas, as estimativas das variāncias e covariâncias das estimativas dos parâmetros são:

$$
\begin{aligned}
\nabla(\hat{a}) & =\frac{s^{2}}{18} ; \\
\nabla(\hat{b}) & =\frac{s^{2}}{36} ; \\
\nabla(c) & =\frac{s^{2}}{12} ; \\
\operatorname{CôV}(a \hat{a}, \hat{c}) & =-\frac{s^{2}}{18} ; \\
\operatorname{CÔV}(\hat{a}, \hat{b}) & =\operatorname{CÔV}(\hat{\sigma}, \hat{c})=0
\end{aligned}
$$

onde $s^{2}=$ QMR.

$$
\text { Para os grupos de ensaios, essas estimativas passam }
$$

a ser:

$$
\begin{aligned}
\nabla(\hat{a}) & =\frac{s^{2}}{18 N} ; \\
\nabla(\hat{b}) & =\frac{s^{2}}{36 N} ; \\
\nabla(\hat{c}) & =\frac{s^{2}}{12 N} ; \\
\operatorname{CôV}(G, \hat{c}) & =-\frac{s^{2}}{18 N} ; \\
\operatorname{CÔV}(\hat{a}, \hat{C}) & =\operatorname{Côv}(6, \hat{c})=0 ;
\end{aligned}
$$


onde $s^{2}$ passa a ser o quadrado médio da interação Ensaios por Nutri ente, jā que, nesse caso, o nitrogênio, o fósforo e o potássio são estudados separadamente e testados em relação a essas interações.

Os intervalos de confiança dos parâmetros dessa regressão, para o caso dos grupos de $\mathrm{N}$ ensaios, serão portanto:

$$
\begin{aligned}
& I C=\left[\hat{a}-0,24 \frac{t s}{\sqrt{N}}, \hat{a}+0,24 \frac{t s}{\sqrt{N}}\right], \\
& I C=\left[6-0,17 \frac{t s}{\sqrt{N}}, \quad 6+0,17 \frac{t s}{\sqrt{N}}\right], \\
& I C=\left[\hat{c}-0,29 \frac{t s}{\sqrt{N}}, \hat{c}+0,29 \frac{t s}{\sqrt{N}}\right],
\end{aligned}
$$

onde $t$ foi tomado ao nível de $95 \%$ de probabilidade e com um nümero de graus de liberdade referente à interação ensaios por nutriente. Para o caso do calcário, as estimativas das variâncias para os grupos de $\mathrm{N}$ ensaios são:

$$
\begin{aligned}
\nabla(\hat{a}) & =\frac{s^{2}}{6 N} ; \\
\nabla(\hat{b}) & =\frac{s^{2}}{12 N} ; \\
V(\hat{c}) & =\frac{s^{2}}{4 N} ; \\
\operatorname{Cov}(\hat{a}, \hat{c}) & =-\frac{s^{2}}{6 N} ; \\
\operatorname{COV}(\hat{a}, \hat{b}) & =\operatorname{COV}(\hat{b}, \hat{c})=0 .
\end{aligned}
$$


Os intervalos de confiança dos parâmetros foram determinados, nesse caso, através de:

$$
\begin{aligned}
& I C=\left[\dot{a}-0,41 \frac{t s}{\sqrt{N}}, \hat{a}+0,41 \frac{t s}{\sqrt{N}}\right], \\
& I C=\left[6-0,29 \frac{t s}{\sqrt{N}}, 6+0,29 \frac{t s}{\sqrt{N}}\right], \\
& I C=\left[\hat{c}-0,50 \frac{t s}{\sqrt{N}}, \hat{c}+0,50 \frac{t s}{\sqrt{N}}\right],
\end{aligned}
$$

onde $s^{2}=Q M(E \times C)$.

\subsubsection{2 - As doses econômicas e seus intervalos de confiança}

Como no caso dos cortes da superficie, facilmente che ga-se à fórmula da dose economicamente aconselhável dos nutrientes (N, $\mathrm{P}_{2} \mathrm{O}_{5}$ e $\mathrm{K}_{2} \mathrm{O}$ ), bem como seus intervalos de confiança para o caso dos grupos de $\mathrm{N}$ ensaios.

$$
\begin{aligned}
x^{*} & =\frac{1}{2 \hat{c}}\left[\frac{t-\hat{E}}{w}\right), \quad \hat{c}<0, \\
\hat{V}\left(x^{*}\right) & \left.=\frac{s^{2}}{48 N \hat{c}^{2}}\left[\frac{\frac{t}{w}-\hat{b}}{\hat{c}}\right)^{2}+\frac{1}{3}\right] .
\end{aligned}
$$

Admitindo-se que a distribuição de $x^{*}$ seja aproximadamente normal:

$$
I C=\left[x^{*}-\operatorname{ts}\left(x^{*}\right), x^{*}+\operatorname{ts}\left(x^{*}\right)\right]
$$


56.

Para o caso do calcário, a expressäo aproximada da variància de $x^{*}$ e seus intervalos de confiança foram determinados através de:

$$
\begin{aligned}
& \left.\hat{V}\left(x^{*}\right)=\frac{s^{2}}{16 N \tilde{c}^{2}}\left[\frac{\frac{t}{w}-6}{\tilde{c}}\right)^{2}+\frac{1}{3}\right], \\
& I C=\left[x^{*}-\operatorname{ts}\left(x^{*}\right), x^{*}+\operatorname{ts}\left(x^{*}\right)\right],
\end{aligned}
$$

e para o cálculo das doses economicamente aconselháveis de calcário, foi considerada a relação: $\frac{w}{t}=10$. 
57.

\section{RESULTADOS E DISCUSSÃO}

Como foi visto no capítulo anterior, os dados utilizados neste trabalho constam de 37 ensaios fatoriais $3^{3}$ de $N$, P e K, com duas repetições, e 34 ensaios em blocos casualizados, com seis repetições, onde o tratamento estudado fol o calcärio, também em três nivveis. Com base nesses ensaios, e considerando os fatores climáticos e edáficos, além das varianncias residuais de cada experi mento, foi possfvel a formação de nove grupos distintos, assim distribuidos:

\begin{tabular}{cc}
\hline Regiões & Grupos \\
\hline Mata & 1 \\
Agreste & $2,3,4,5,6$ \\
Sertão & $7,8,9$ \\
\hline
\end{tabular}


As anälises individuais e conjuntas foram feitas segundo os esquemas mostrados em 4.2.2. Os desvios padrão, coeficientes de variação e as análises estatísticas, por elemento, se encontram nas Tabelas 3 a 6 , do apêndice.

Pelas Tabelas 3 e 4 observa-se a grande amplitude dos coeficientes de variação dos ensaios individuais, notadamente para os ensaios fatoriais que variaram de $12,4 \%$ a $45,1 \%$. Entretanto, com a formação dos grupos, não só esses coeficientes se tornaram mais homogêneos, como se situaram numa faixa tida como aceitável.

Os resultados mostrados nas Tabelas 5 e 6 ,demonstram uma predomináncia do efeito do nitrogênio, sendo significativo em 7 dos 9 grupos e, na maioria, ao nível de 1\% de probabilidade. Em segundo lugar colocaram-se o fósforo e o potássio com apenas dois casos favoráveis, sendo, entretanto, o efeito do fósforo mais acentua do que o do potássio. Quanto ao calcário, dos 8 grupos estudados, reagiu em apenas 2 casos e mesmo assim com valores de $F$ observados bem próximos dos teóricos, ao nível de $5 \%$ de probabilidade. E interessante salientar que o acréscimo da produção da dose 2 de calcárío, em relação à testemunha, foi muito pequeno, sendo, inclusive, negativo em alguns casos.

Estes resultados concordam com os obtidos por PIMENTEL GOMES e CAMPOS (1966) quando afirmam haver uma predominância do efeito do nitrogênio sobre o fósforo, o potássio e o calcário, nos diversos tipos de solos e em diferentes regiões brasileiras. De uma 
certa forma, as outras conclusões obtidas pelos autores foram também concordantes com as do presente trabalho.

\section{1 - O Ajustamento da Regressão Polinomial Quadrātica}

\section{1 .1 - Resultados e discussão dos parâmetros e seus res" pectivos intervalos de confiança}

As estimativas dos parâmetros da regressão polino-

mial quatrática, segundo a teoria desenvolvida em 4.2.3.1 são obtidas com grande facilidade, sendo necessário apenas dispor dos quadros das interações. As estimativas dos parâmetros dos ensaios individuais e dos grupos de ensaios, bem como seus intervalos de confiança, ao nível de $95 \%$ de probabilidade, podem ser vistos nas Tabe las 7 e 8 , do apêndice.

Em um rápido exame dessas tabelas, observa-se a gran de amplitude dos intervalos de confiança dos parâmetros, mesmo para os grupos de ensaios onde a variação deveria ser teoricamente bem menos acentuada.

No caso dos grupos de ensaios, com exceção do parâme tro $a_{0}$, que apresentou intervalos de confiança com amplitudes varian do de $10 \%$ a $40 \%$ do valor de suas estimativas, os demais paràmetros, em sua grande maioria, apresentaram intervalos de grandes amplitudes e, em alguns casos, até absurdos, principalmente os de estimati vas próximas de zero, que poderiam assumir valores positivos e nega 
60.

tivos dentro do intervalo de confiança considerado, fato esse que, para os coeficientes dos termos quadráticos, significa a reversão de tendência para máximo ou para mínimo ou para ponto de sela.

O teste $t$ dos parâmetros das regressões, para o caso dos grupos de ensaios, apresentou os seguintes resultados:

\begin{tabular}{|c|c|c|c|c|c|c|c|c|c|c|}
\hline \multirow{2}{*}{ GRUPOS } & \multicolumn{10}{|c|}{ PARÂMETROS } \\
\hline & $a_{0}$ & $a_{11}$ & $a_{22}$ & $a_{33}$ & $a_{12}$ & $a_{13}$ & $a_{23}$ & $a_{14}$ & $a_{24}$ & $a_{34}$ \\
\hline 1 & 9,58 & 1,78 & $2,4 \stackrel{\leftrightarrow}{6}$ & 2,59 & 1,17 & 1,95 & 0,20 & $4,63^{\text {sitk }}$ & 1,66 & 0,48 \\
\hline 2 & $26,84^{\% \%}$ & 2,41 & 2,13 & 0,01 & $3,17^{* *}$ & 0,32 & $2,5 \frac{2}{6}$ & $22,87^{* * *}$ & $6,2^{* * *}$ & 0,76 \\
\hline 3 & $36,38^{* * 6}$ & 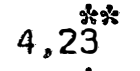 & 0,91 & 1,91 & 1,74 & 0,52 & 0,41 & $13,50^{* \%}$ & $3,87^{* *}$ & 1,54 \\
\hline 4 & $20,68^{* \%}$ & 2,33 & $4,13^{k *}$ & 0,52 & 5,65 & $2.93^{x}$ & 0,49 & $12,1 \frac{*}{4}$ & $11,10^{*}$ & 1,70 \\
\hline 5 & $30,72^{20 \%}$ & $7,26^{* \%}$ & $4,40^{* * *}$ & $3,38^{\circ \%}$ & $4,19^{* *}$ & 0,05 & 1,35 & $22,88^{* * *}$ & $9,73^{*}$ & $3,00^{* * \pi}$ \\
\hline 6 & $30,04^{20 \% \text { ? }}$ & 1,90 & $2,70^{* * *}$ & 0,82 & 0,95 & 0,54 & 1.67 & $6,50^{\circ 8}$ & $6,33^{* *}$ & 1,29 \\
\hline 7 & $25,3 i^{* * *}$ & 0,48 & 1.16 & 1,20 & 0,58 & 0,77 & 2.39 & $8,03^{\frac{*}{*} \%}$ & 0,31 & 1,25 \\
\hline 8 & $27,30^{* 6 \%}$ & $3,62^{* \% *}$ & 0,13 & 0,03 & 0,28 & 1,98 & 0,62 & $2,0 \ddot{\theta}$ & 0,84 & 0,25 \\
\hline 9 & $23,07^{\circ}$ & $3,29^{* 2 \%}$ & 0,42 & $3,81^{8 * 8}$ & 0,32 & 1,75 & 1,37 & $18,38^{k+k}$ & 1,12 & 1,18 \\
\hline
\end{tabular}

o quadro acima nos mostra a predominância dos efeitos dos parâmetros dos termos lineares em relação aos dos termos qua dráticos e das interações, sendo significativos em quase sua totali dade, ao nivel de $1 \%$ de probabilidade. Os coeficientes dos termos quadräticos também apresentaram värios casos significativos, se bem que menos acentuadamente, enquanto que, os coeficientes dos termos 
das interações apresentaram poucos casos. Talvez a exclusão desses termos do modelo, já que são de pouco monta, não influa no ajustamento aos dados, como observaram CAMPOS e ARAUJO (1971), o que nos permite sugerir, para estudos posteriores, a exclusão desses parâme tros da regressão. Essa recomendação é reforçada pelas conclusões ob tidas por CAMPOS e PIMENTEL GOMES (1976), quando afirmam serem as interações lineares $N^{\prime} \times P^{\prime}, N^{\prime} \times K^{\prime}$ e $P^{\prime} \times K^{\prime}$ de importância secundáría e. na maioria dos casos, de pouca monta se comparadas aos efeitos principais, quando se consideram doses moderadas de nutrientes, como tivemos oportunidade de verificar.

\section{1 .2 Os intervalos de confiança das estimativas dos ren dimentos}

Através da teoria desenvolvida em 4.2.3.2, as estima tivas dos rendimentos dos 27 tratamentos fatoriais dos grupos de en saios foram determinadas comos mostra a Tabela 12 do apêndice. Nessa Tabela, também foram determinados os intervalos de confiança des ses rendimentos, ao nivel de $95 \%$ de probabilidade, permitindo-nos ti rar as seguintes conclusões:

1) A imprecisão com que são determinados os parâmetros reflete-se na dos rendimentos, embora menos acentuadamente, em virtude das expressões que determinam as variâncias para cada tratamento. A medida que os grupos se tornam mais numerosos, essas variâncias vão decrescendo, implicando em intervalos de confiança mais curtos. 
Outro fator que influi na grandeza dessa dispersão, é a variância média residual dos grupos que, por sua vez, depende da dos ensaios individuais que as compōem. Essas conclusões concordam plenamente com as obtidas por CAMPOS (1967) quando afirma ser recomendada a re gressão polinomial apenas para grupos de ensaios de boa precisão.

2) Os intervalos de confiança das estimativas dos rendumentos, de uma maneira geral apresentaram amplitudes superiores a $10 \%$ desses rendimentos, resultado esse que discorda dos conseguidos por COSTA (1977), que obteve intervalos bem mais curtos. 0 fato provavelmente se deve à pequena variância residual obtida por esse autor para o grupo que tomou como exemplo.

3) Na grande maioria dos casos, as produções observadas situaram-se dentro do intervalo de confiança, mais acentuadamente, entre tanto, nos casos onde os coeficientes de determinação total foram altos.

4) Não foram observados, para os grupos de ensaios, intervalos de confiança que permitissem estimativas negativas para os rendimen tos. Entretanto, a hipötese de obtê-las não deve ser descartada,prin cipalmente nos casos onde a variància residual seja bastante alta e os rendimentos baixos.

5) Em vários casos, os rendimentos observados e estimados foram bastante discrepantes, reflexos, sem dúvida, da imprecisão com que foram determinadas as estimativas dos parâmetros da regressão. 
Lcg 3, a estimação dos rendimentos através dessa superfície deve ser olhada com uma certa reserva.

$\begin{aligned} 5.1 .3- & \text { As doses ötimas de } N, P_{2} O_{5} \text { e } K_{2} O \text { e discussão do mä } \\ & \text { ximo }\end{aligned}$

A solução do sistema de equações:

$$
\left\{\begin{array}{l}
2 \hat{a}_{11} x_{1}+\hat{a}_{12} x_{2}+\hat{a}_{13} x_{3}=t_{1} / w-\hat{a}_{14} \\
\hat{a}_{12} x_{1}+2 \hat{a}_{22} x_{2}+\hat{a}_{23} x_{3}=t_{2} / w-\hat{a}_{24} \\
\hat{a}_{13} x_{1}+\hat{a}_{23} x_{2}+2 \hat{a}_{33} x_{3}=t_{3} / w-\hat{a}_{34}
\end{array},\right.
$$

pode nos conduzir a uma receita líquida mäxima, se as seguintes con diçōes forem satisfeitas:

1) $\Delta_{1}<0$

2) $\frac{\Delta_{2}}{\Delta_{1}}<0$

onde:

$$
\begin{aligned}
& \Delta_{1}=2 \hat{a}_{11} \\
& \Delta_{2}=\left|\begin{array}{ll}
2 \hat{a}_{11} & \hat{a}_{12} \\
\hat{a}_{12} & 2 \hat{a}_{22}
\end{array}\right| \\
& \Delta_{3}=\left|\begin{array}{lll}
2 \hat{a}_{11} & \hat{a}_{12} & \hat{a}_{13} \\
\hat{a}_{12} & 2 \hat{a}_{22} & \hat{a}_{23} \\
\hat{a}_{13} & \hat{a}_{23} & 2 \hat{a}_{33}
\end{array}\right|
\end{aligned}
$$


Segundo CAMPOS (1967), uma condição necessária para que a função de recelta líquida, no ponto $\left(x_{1}^{+}, x_{2}^{+}, x_{3}^{+}\right)$, seja máxima, é que os coeficientes $\hat{a}_{11}, \hat{a}_{22}$ e $\hat{a}_{33}$ sejam todos negativos. Então, consequentemente, para que essa função admita um mínimo no ponto considerado é necessário que esses mesmos coeficientes sejam todos positivos.

As soluções dos sistemas de equaçöes, bem como os ele mentos da matriz diagonal $D_{\text {, }}$ para os nove grupos de ensaios, estão na Tabela 13 do apêndice. Além desses casos, foi verificedo, também, a dos ensaios individuais que apresentaram coeficientes: $\hat{a}_{11}, \hat{a}_{22}$, $\hat{a}_{33}$, de mesmo sinal, e que poderiam, portanto, dar pontos de máximo ou de mínimo.

Num rápido balanço dos resultados e conclusões obtidas nesse parägrafo temos:

1) Para os grupos de ensaios, todos os pontos obtidos foram de sela. Algumas justificativas podem ser dadas ao fato, tais como:

a) A pouca tendência ao efeito quadrático negativo, simulta neamente para o nitrogênio, o fósforo e o potássio, excluindo, de início, a possibilidade de obtenção do máximo para os grupos:

b) Sendo as estimativas dos parâmetros da regressão para os grupos de ensaios a média aritmética dos respectivos parâmetros estimados dos ensaios individuais, seria necessário, portanto, que a soma algébrica dos coeficientes de 
cada termo quadrätico fosse negativa. Assim, se observar mos, por exemplo, os coeficientes dos termos quadräticos dos ensalos que compöem o grupo 3, vamos notar que todos os seis coeficientes $\hat{a}_{3}$ são positivos, excluindo, de inf́cio, a obtenção do máximo nesse caso. Alıás, o efeito quadrático positivo e bem acentuado do potássio foi observado na grande maioria dos casos, dificultando a obtenção de uma receita liquida máxima para as soluções en contradas,

c) O nümero reduzido de ensalos dos grupos.

2) Dos oito ensaios individuais, cujos coeficientes $\hat{a}_{11}, \hat{a}_{22} e$ $\hat{a}_{33}$ foram negativos, seis deles nos conduziram, de fato, a uma receita líquida máxima, como pode ser observado no quadro abaixo.

\begin{tabular}{crrrrrr}
\hline Ensaios & $\begin{array}{c}N \\
(\mathrm{~kg} / \mathrm{ha})\end{array}$ & $\begin{array}{c}\mathrm{P}_{2} \mathrm{O}_{5} \\
(\mathrm{~kg} / \mathrm{ha})\end{array}$ & $\begin{array}{c}\mathrm{K}_{2} \mathrm{O} \\
(\mathrm{kg} / \mathrm{ha})\end{array}$ & $\Delta_{1}$ & $\Delta_{2} / \Delta_{1}$ & $\Delta_{3} / \Delta_{2}$ \\
\hline 2.1 & 175,9 & $-124,8$ & $-127,9$ & $-577,0$ & $-512,2$ & $-121,1$ \\
2.4 & 102,9 & 123,1 & 66,2 & $-696,2$ & $-73,7$ & $-9,8$ \\
6.1 & 93,3 & $-315,5$ & $-42,7$ & $-583,4$ & $-88,7$ & $-164,6$ \\
8.4 & $-47,7$ & $-147,8$ & $-33,6$ & $-500,8$ & $-167,0$ & $-330,5$ \\
8.5 & 31,3 & 13,9 & 23,5 & $-1364,4$ & $-322,4$ & $-204,8$ \\
9.3 & 176,6 & 26,5 & 27,2 & $-491,0$ & $-464,3$ & $-1036,0$ \\
\hline
\end{tabular}


Desses, o ensaio 8.5, lançado em Petrolândia em 1974, foi o único que apresentou doses econômicas dentro do intervalo pes quisado, ou seja: $31,3 \mathrm{~kg} / \mathrm{ha}, 13,9 \mathrm{~kg} / \mathrm{ha}$ e $23,5 \mathrm{~kg} / \mathrm{ha}$ de $\mathrm{N}, \mathrm{P}_{2} \mathrm{O}_{5}$ e $\mathrm{K}_{2} \mathrm{O}$ respectivamente.

3) Não foram observadas soluções que conduzissem a uma receita líquida mínima.

\section{1 .4 - Resultados e discussão dos cortes dados na super ficie}

Através da teoria desenvolvida em 4.2.3.4, foram estudados o comportamento de cada variável quando fixadas a duas outras, em suas doses médias. As doses economicamente aconselháveis dos nutrientes: nitrogènio, fósforo e potássio, bem como os interva los de confiança, ao nível de 95\% de probabilidade, estão na Tabela 14 do apêndice.

Como se pode deduzir dessa Tabela, dos 17 casos em que a dose $x^{20}$ nos conduziu a uma receita líquida máxima, 14 deleses tão situados dentro da faixa explorada pelo ensaio. Desses 14 casos, 9 apresentaram intervalos de confiança que também não extrapolaram essa faixa, sendo portanto viável na maioria dos casos a recomendação com base nesses resultados, levando-se em conta, é claro, as im perfeições do método de estimação e do número reduzido de dados com que foram determinadas essas doses, não devendo, portanto, serem tó madas como definitivas as conclusões a que chegamos e sim, como ori 
entação para trabalhos subsequentes, principalmente se considerarmos as amplitudes dos intervalos de confiança das doses obtidas, que só em poucos casos foram razoáveis.

No quadro a segu1r apresentamos um resumo dessas recomendações: onde a linha pontilhada significa que a dose obtida extrapolou o intervalo pesquisado.

\begin{tabular}{lccc}
\hline \multicolumn{1}{c}{ Municípios } & $\begin{array}{c}\mathrm{N} \\
(\mathrm{kg} / \mathrm{ha})\end{array}$ & $\begin{array}{c}\mathrm{P}_{2} \mathrm{O}_{5} \\
(\mathrm{~kg} / \mathrm{ha})\end{array}$ & $\begin{array}{c}\mathrm{K}_{2} \mathrm{O} \\
(\mathrm{kg} / \mathrm{ha})\end{array}$ \\
\hline $\begin{array}{l}\text { Vitória de Santo Antão } \\
\text { Glória do Goitá e Limoeiro }\end{array}$ & mínimo & 17,3 & mínimo \\
Surubim e Vertentes & 51,6 & 34,2 & zero \\
Timbaüba e Vicência & 71,0 & 69,7 & 29,0 \\
Caruaru & 73,6 & 54,3 & mínimo \\
Bom Conselho e Garanhuns & 20,1 & 33,4 & mínimo \\
Serra Talhada & $\mathrm{minimo}$ & 28 ro & mínimo \\
Belém do Säo Francisco e Petroländia & 33,7 & minimo & mínimo \\
Tacaratu & 96,5 & mínimo & 25,2 \\
\hline
\end{tabular}

Os Intervalos de conflança das doses economicamente aconselháveis para o nitrogênio, o fásforo e o potássio, foram determinados mesmo para os casos onde os coeficientes dos termos quadräticos não diferiram significativamente de zero, apesar de que não se deva proceder de tal forma nesses casos (D'AULISIO, 1976). Um re sultado que elucida muito bem a impropriedade de tal procedimento, 
68.

fol o da dose de potássio do grupo 2, cujo valor de $t$ do parâmetro a 3 foi de 0,099, valor este muito baixo e não significativo, implicando na absurda amplitude de $9250,4 \mathrm{~kg} / \mathrm{ha}$ para o intervalo de confiança, conforme se verifica na Tabela 14 do apêndice. Por outro lado, foram razoáveis os intervalos de confiança das doses de nitró gênio dos grupos 3 e 5 e do fósforo do grupo 4 , justamente os valores de $t$ mais altos e significativos ao nivel de $1 \%$ de probabilidade.

\section{1 .5 - Valores de $F$ das regressöes e dos desvios das re- gressões,e coeficientes de determinação total}

De 4.2.3.4 obteve-se a seguinte expressão que deter mina a soma de quadrados da regressão:

$S Q \operatorname{Reg}=S Q N+S Q P+S Q K+S Q N^{\prime} \times P^{\prime}+S Q N^{\prime} \times K^{\prime}+S Q P^{\prime} \times K^{\prime}$,

e. consequentemente, os valores de F da regressão e dos desvios da regressão, são obtidos respectivamente através de:

$$
\frac{\text { QM Regressão. }}{\text { QM Resíduo }} \quad \text { QM Desvios da Regressão. }
$$

onde: SQ Desvios da Regressão = SQ Tratamentos - SQ Regressão.

Os coeficientes de determinação total, para o caso presente, foram obtidos através da seguinte relação:

$$
R^{2}=\frac{S Q \text { Regressão }}{S Q \text { Tratamentos }} .
$$


69.

Em uma rápida análise na Tabela 11 (Apêndice), onde foram determinados os valores de $F$ das regressöes e dos desvios das regressões, bem como os coeficientes de determinação total, temos:

1) Para os grupos de ensaios, com exceção do 1 e do 8 , o ajustamento pode ser considerado satisfatório, uma vez que apresentaram valores de F significativos ao nível de $1 \%$ de probabilidade, teste F para os desvios da regressão não significativo e coeficientes de determinação total entre $80 \%$ e $95 \%$.

2) Os grupos 2 e 5 foram os que apresentaram melhores resultados quanto ao ajustamento, com valores de $F$ bastante significativos e coeficientes de determinação total entre $94 \%$ e $95 \%$. A mesma coisa, entretanto, não pode ser dita com relação aos grupos 1 e 8 , apesar de não terem apresentado valores de $F$ para os desvios da regressão significativos ao nível de $5 \%$ de probabilidade.

3) Os ensaios individuais não apresentaram valores de F significativos para os desvios da regressão, com exceção do 4.1 e 5.4 . mesmo assim, com $F$ para regressão altamente significativo.

4) Observou-se que os valores de F das regressões e dos desvios da regressão, bem como dos coeficientes de determinação total dos grupos, situam-se próximos dos obtidos para os ensaios individuais que os compöem. Assim, quanto melhor for o ajustamento dos en saios individuais que compõem um determinado grupo, mais afinidade existe entre ele e o modelo. 
70.

\section{2 - O Uso do Trinômio do Segundo Grau}

Os valores das estimativas dos paràmetros e respecti vos desvios padrão do trinômio do segundo grau, para nitrogênio,fós foro, potássio e calcário, estão contidos nas Tabelas 9 e 10 do apêndice. Da comparação dessas estimativas com aquelas obtidas para os cortes da superffcie, de acordo com 4.2.4.1, chegou-se às seguin tes conclusões:

a) Para o nitrogênio: $b=\hat{a}_{14}$,

$$
\hat{c}=\hat{a}_{11} ;
$$

b) Para o fósforo: $\quad G=\hat{a}_{24}$,

$$
\hat{c}=\hat{a}_{22}:
$$

c) Fara o potássio: $\quad G=\hat{a}_{34}$.

$$
\tilde{c}=\tilde{a}_{33} .
$$

Em decorrência disso, as fórmulas que estimam as doses economicamente aconsel háveis de nitrogênio, fósforo e potássio passam a ser iguais em ambos os casos, sendo, portanto, desnecessária a sua determinação pelo trinômio do segundo grau. Para o calcário, entretanto, foram determinadas essas doses, bem como seus intervalos de confiança ao nfvel de $95 \%$ de probabilidade, através das expressões deduzidas em 4.2.4.2. Os resultados obtidos para os grupos de ensaios constam da Tabela 15 do apêndice, de onde tirou-se as seguintes conclusões: 
1) Em nenhum caso, as doses ótimas de calcário ultrapassaramos 2000 kg/ha, não sendo necessário, portanto, a recomendação por extra polação.

2) Parece ser razoável a recomendação de $1000 \mathrm{~kg} / \mathrm{ha}$ de calcário para os locais que constituem os grupos 1, 3, 4 e 6, principalmente porque será necessário apenas um acréscimo de produção da ordem de $100 \mathrm{~kg} / \mathrm{ha}$ de milho em grão para compensar os gastos com esse corretivo, considerando-se os preços que foram tomadas por base nes te trabalho e sem considerar a mão-de-obra.

3) Para os municípios de Glória do Goitá e Limoeiro, a calagem não é recomendada, uma vez que a dose obtida foi negativa.

4) Mesmo considerando que os coeficientes dos termos quadráticos não diferiram significativamente de zero, as recomendações feitas para o calcário não se invalidam, dado o baixo preço desse corretivo. 
72.

\section{CONCLUSÕES}

Do presente trabalho, pode-se concluir, de uma mane1

ra geral:

1 - Dos 9 grupos de ensaios em 14 municípios da Zona da Mata ao Sertão, apenas em Serra Talhada e Tacaratu o efeito do nitrogènio não foi significativo. Em alguns casos, o aumento de produção da dose 2 em relação à ausência de nitrogênio foi de quase $2000 \mathrm{~kg} / \mathrm{ha}$. Tudo leva a crer que há uma grande predominância do efeito desse nu triente sobre o do fósforo, do potássio e do calcário, em todos os tipos de clima e solo, onde se cultiva o milho, no Estado de Pernambuco.

2 - De uma maneira geral, os parâmetros da regressão polinomial quadrätica para os grupos de ensaios apresentaram intervalos de con fiança bastante amplos $e$, em alguns casos, até absurdos, principal 
mente os de estimativas próximas de zero, que poderiam assumir valo res positivos ou negativos dentro de seus intervalos. Neste caso,pa ra os coeficientes dos termos quadráticos, significa a reversão de tendência para máximo ou para mínimo, ou para ponto de sela.

3-Raramente os parâmetros das interações do modelo polinomial quadrático apresentaram significância estatística. Por isso, talvez fosse interessante, em estudo posterior, a exclusão desses termos do modelo com a finalidade de se verificar o seu comportamento.

4 - A imprecisão com que são determinados os parâmetros da regressão polinomial quadrática, reflete-se na dos rendimentos, embora menos acentuadamente.

5 - Na maioría dos casos, as produções observadas situaram-se dentro do intervalo de confiança dos rendimentos, mais acentuadamen te nos casos onde os coeficientes de determinação total foram altos.

6 - Com exceção dos grupos 1 e 8 , o ajustamento da superfície de resposta aos dados pode ser considerado como razoável.

7 - Para os grupos de ensaios, todos os pontos obtidos da solu ção do sistema de equações das derivadas parciais de primeira ordem da função de receita líquida foram de sela.

8 - As doses economicamente aconselháveis de $\mathrm{N}_{3} \mathrm{P}_{2} \mathrm{O}_{5}$ e $\mathrm{K}_{2} \mathrm{O}$, ob tidas com os cortes da superfície, são as que constam do quadro seguinte, onde a linha pontilhada significa que a dose obtida extrapo lou o intervalo pesquisado. 


\begin{tabular}{|c|c|c|c|}
\hline Munteipios & $\begin{array}{c}N \\
(\mathrm{~kg} / \mathrm{ha})\end{array}$ & $\begin{array}{c}\mathrm{P}_{2} \mathrm{O}_{5} \\
(\mathrm{~kg} / \mathrm{ha})\end{array}$ & $\begin{array}{c}\mathrm{K}_{2^{0}} \\
(\mathrm{~kg} / \mathrm{ha})\end{array}$ \\
\hline Vitöria de Santo Antäo & mínimo & 17,3 & minimo \\
\hline Glória do Goitá e Limoeiro & $\cdots$ & 34,2 & zero \\
\hline Surubim e Vertentes & 51,6 & zero & minimo \\
\hline Timbauba e Vicência & 71,0 & 69,7 & 29,0 \\
\hline Caruaru & 73,6 & 54,3 & minimo \\
\hline Bom Conselho e Garanhuns & 20,1 & 33,4 & mInimo \\
\hline Serra Talhada & minimo & zero & mínimo \\
\hline Belém do São Francisco e Petrolándia & 33.7 & mínimo & mínimo \\
\hline Tacaratu & 96,5 & mínimo & 29,2 \\
\hline
\end{tabular}

9 - As doses economicamente aconselháveis de nitrogênio, fósfo ro e potássio, são as mesmas nos cortes da superfície e no trinômio do segundo grau, no caso da codificação da variável $x_{1}$ em -1, 0 e 1. 10 - As doses ótimas de calcário, obtidas pelo trinómio do segundo grau, indicaram como razoävel a quantidade de $1000 \mathrm{~kg} / \mathrm{ha}$ des se corretivo para os grupos $1,3,4$ \& 6 , enquanto que para o grupo 2 a calagem näo é recomendada. Para os demais grupos, as doses $x^{*}$ nos conduziram a uma receita líquida mínima.

11 .. A obtenção do mäximo, através da regressão polinomial quadrática, torna-se problemätica quando os grupos são constituídos de poucos ensaios a suas precisões deIxam muito a desejar, como fol o caso do presente trabalio. 
12 - Os intervalos de confiança para a dose economicamente recomendável, calculados em casos em que o coeficiente do termos quadrático näo diferiu significativamente de zero, deram resultados ab surdos, o que comprova ser esse cálculo desaconselhável nessas circunstàncias.

13 - As recomendações feitas neste trabalho não merecem muita confiança, em virtude do pequeno número de ensaios de que dispuzemos, näo devendo, portanto, serem encaradas como definitivas. Entre tanto, dão, sem dúvida, uma boa indicação para trabalhos subsequentes. 
76.

\section{SUMMARY}

Having in view recommendations for the fertilization of maize in the State of Pernambuco (Brasil), $373^{3}$ factorial experiments with $N, P$ and $K$, each with 2 replications with confounding (group $w$ ). Three additional treatments were included for limestone in each block of 6 plots.

Nine groups of trials were considered, including 14 counties and 3 ecological regions.

The following quadratic polynomial was fitted to the data of each group:

$$
\begin{aligned}
& Y=a_{0}+a_{11} x_{1}^{2}+a_{22} x_{2}^{2}+a_{33} x_{3}^{2}+a_{12} x_{1} x_{2}+a_{13} x_{1} x_{3}+a_{23} x_{2} x_{3}+a_{14} x_{1}+ \\
& +a_{24} x_{2}+a_{34} x_{3} . \\
& \text { The following topics were studied: }
\end{aligned}
$$


1. Estimation and testing of the parameters, and calculation of confidence intervals for them.

2. Confidence intervals for the calculated yields.

3. Optimum dressings of $\mathrm{N}_{2} \mathrm{P}_{2} \mathrm{O}_{5}$ and $\mathrm{K}_{2} \mathrm{O}$.

4. Section of the response surface by planes parallel to the coordinated axes.

5. Coefficient of determination.

When dealing with limestone, the optimum dressing was obtained with the aid of a one-variable second degree polynomial. The main conclusions obtained were:

1. Confidence intervals for regression polynomials parameters were very wide and sometimes absurd. The calculated yield were too variable also, even if not as much as the estimates of the parameters.

2. Parameters refferring to the interaction terms were not signigicant, suggesting their elimination in future cases.

3. The response surfaces obtained were considered acceptable, except in groups 1 and 8 .

4. Sections of the response surface led to the following recommendations. 


\begin{tabular}{cccc}
\hline Groups & $\mathrm{N}(\mathrm{kg} / \mathrm{ha})$ & $\mathrm{P}_{2} \mathrm{O}_{5}(\mathrm{~kg} / \mathrm{ha})$ & $\mathrm{K}_{2} \mathrm{O}(\mathrm{kg} / \mathrm{ha})$ \\
\hline 1 & Minimum & 17.3 & Minimum \\
2 & $\ldots$ & 34.2 & Zero \\
3 & 51.6 & Zero & Minimum \\
4 & 71.0 & 69.7 & 29.0 \\
5 & 73.6 & 54.3 & Minimum \\
6 & 20.1 & 33.4 & Minimum \\
7 & Minimum & 2 Ero & Minimum \\
8 & 33.7 & Minimum & Minimum \\
9 & 96.5 & Minimum & 29.2 \\
\hline
\end{tabular}

The dots indicate a solution with dangerous extrapolation.

5. Confidence intervals for the optimum dressing calculated in cases where the coefficient of the quadratic term was non-significant gave absurd results, which shows that this procedure is not advisable in such cases.

6. For groups 1, 3, 4 and 6 the use of $1000 \mathrm{~kg} . /$ ha of limestone is advisable, while for group 2 imestone should not be applied, For the other group there was a minimum, instead of a.maximum.

7. Quadratic polynomial regression, as expected, was not convenient to detect optimum dressings in groups of a few trials of low precision. 
79.

8. Recommendations showed in this paper are not very reliable, since they were obtained from a small number of experiments. However they may be good suggestions for future research. 
80.

\section{BIBLIOGRAFIA}

BDX, G.E.P. e K.B. WILSON, 1951. On the Experimental Attainment of Optimum Condictions. Journ. Roy. Stat. Soc. Série B. 13: 1-45.

CAMPOS, Ho, 1967. Aspectos da Aplicação das Superfícles de Resposta a Ensaios Fatoriais $3^{3}$ de Adubação. Piracicaba. 82 pp. (Tese de Livre-Docência).

CAMPOS, H. e P.C. ARAUJJ, 1971. Aspectos da Adubação do Milho. Departamento de Ciências Sociais Aplicadas, ESALQ/USP. Piracicaba. $41 \mathrm{pp}$.

CAMPOS, H. e F. PIMENTEL GOMES (1976). As Interações em Grupos de Experimentos de Adubação de Milho. Rev.Agricultura. Piracicaba, 51: 5-16.

COCHRAN, W.G. e G.M. COX, 1957. Experimental Designs. 3. ed. Nova York. John Wiley and Sons. 611 pp. 
COSTA, R.A., 1977. Funções de Produção Ajustadas a Ensaios Fatoriais $3^{3}$ de Adubação de Arroz. Piracicaba. 80 pp. (Dissertação de Mestrado).

D'AULISIO, M.de B.G., 1976. A Variāncia dos Pontos de Máximo ou de MÍnimo de Equações de Regressão de Segundo Grau. Piracicaba. 61 pp. (Dissertação de Mestrado).

FUNDAÇÃO INSTITUTO BRASILEIRO DE GEOGRAFIA E ESTATISTICA, 1976. Anuá rio Estatístico Brasileiro. Rio de Janeiro. 813 pp.

HEADY, E.0. e J.T. PESEK, 1954. A Fertilizer Production Surface with Specification of Economic Optima for Corn Grown on Calcareous Ida Silt. Loam. Jorn. Farm. Econ. Philadelphia, Penn, 36: 466-482.

MINISTERIO DA AGRICULTURA E MINISTERIO DO INTERIOR, 1974/75. Levan tamento Exploratório - Reconhecimento de Solos do Estado de Pernambuco, $2^{8}$ Volume. Recife.

MORAES, R.S. 1969. Superfĺcies de Resposta num Ensaio Fatorial de Adubação com Níveis Não-Equidistantes. Piracicaba. 58 pp. (Tese de Doutoramento).

NEVES, D.S. e outros, 1960. Ensaios de Adubação do Algodoeiro. Boletim $n^{8} 114$ do Instituto Agronómico de Campinas. Campinas. 33 pp.

OLIVEIRA, L.Mo, 1974. Eficiência de Delineamentos Para Ajustamento de Superflcies de Resposta. Piracicaba. 61 pp. (Dissertação de Mestrado).

PERLIS, S., 1958. Theory of Matrices. 3? ed. Cambridge, Mass. Addr son-Wesley Publishing. $237 \mathrm{pp}$. 
PIMENTEL GOMES, F., 1953. The Use of Mitscherlich's Law in the Analysis of Experiments with Fertilizers. Biometrics, Carolina do Norte, 9: 498-517。

PIMENTEL GOMES, F., 1957. Análise Conjunta de 38 Experimentos de Adubação de Cana-de-Açūcar. Rev. Agricultura. Piracicaba, 52 : 113-126.

PIMENTEL GOMES, F。e I.R. NOGUEIRA, 1964. Regressão e Covariância (mimeografado). Piracicaba. $45 \mathrm{pp}$.

PIMENTEL GOMES, F。 e H. CAMPOS, 1966. Resultados de Ensaios de Adu bação de Milho. In: Cultura e Adubação do Milho. Instituto Bra sileiro de Potassa, pp. 429-449.

PIMENTEL GOMES, F: 1969. Novos Aspectos do Estudo Econômico de Ensaios de Adubação. Fertilité. Paris, 34: 3-21.

PIMENTEL GOMES, F.; 1976. Curso de Estatística Experimental. 6a Edição. Livraria Nobel. Piracicaba. 430 pp.

RAMIREZ DAVILA, L。E。e J. BENITEZ, 1975. Vantajas y Desvantajas del Modelo Polinomial Cuadrático em el Estudio de la Respuesta a los Fertilizantes (mimeografado). Apresentado na Reunião Comemorativa do $20^{\circ}$ Aniversário da Região Brasileira da Sociedade Internacional de Biometria ("The Biometric Society"). 9 pp.

RAO, C.R., 1952. Advanced Statistical Methods in Biometric Research. Nova York. John Wiley, 390 pp.

VADLAMUDI, Y.R。 e H.U. THIMM, 1974. Economic Analysis of Fertilizer Trials Conducted in Kenya on Maize. E.A. Agric. For. J. Nairobi, 40(2): $189-201$. 
83.

VIEIRA, S., 1970. Aspectos das Funções de Produção Ajustadas aos En saios Fatoriais $3^{3}$ de Adubação. Piracicaba. $165 \mathrm{pp}$. (Tese de Dou toramento).

WANDERLEY, M.E., 1971. Aspectos da Adubação do Milho no Estado de Pernambuco. Piracicaba. 67 pp. (Dissertação de Mestrado). 
84.

9. APENDICE 
Tabela 1 - Produção, em kg/ha, referente aos 37 ensaios fatorials de adubação de milho, em diversas regiōes do Estado de Pernambuco.

Grupo 1

\begin{tabular}{|c|c|c|c|c|}
\hline \multirow{2}{*}{ TRATAMENTOS } & \multicolumn{2}{|c|}{ ENSAIO 1.1} & \multicolumn{2}{|c|}{ ENSAIO 1.2} \\
\hline & $\begin{array}{r}1: a \\
\text { Rep. }\end{array}$ & $\begin{array}{r}2 ! \\
\text { Rep. }\end{array}$ & $\begin{array}{c}1_{0}^{a} \\
\text { Rep. }\end{array}$ & $\begin{array}{r}2 ! \\
\text { Rep. }\end{array}$ \\
\hline 000 & 2056 & 500 & 1667 & 778 \\
\hline 010 & 2944 & 1722 & 3611 & 2500 \\
\hline 020 & 1369 & 1556 & 1278 & 2222 \\
\hline 001 & 222 & 2222 & 1278 & 3611 \\
\hline 011 & 111 & 444 & 1667 & 2500 \\
\hline 021 & 222 & 500 & 667 & 667 \\
\hline 002 & 556 & 2167 & 2222 & 2111 \\
\hline 012 & 1556 & 3600 & 2111 & 3000 \\
\hline 022 & 1667 & 944 & 2333 & 2111 \\
\hline 100 & 2000 & 944 & 2222 & 1333 \\
\hline 110 & 1111 & 2722 & 1945 & 2500 \\
\hline 120 & 611 & 2556 & 1278 & 2778 \\
\hline 101 & 556 & 1278 & 1000 & 1556 \\
\hline 111 & 833 & 2111 & 2389 & 2222 \\
\hline 121 & 1833 & 1516 & 1278 & 2111 \\
\hline 102 & 1722 & 2444 & 1556 & 2945 \\
\hline 112 & 1000 & 2167 & 1389 & 2389 \\
\hline 122 & 2833 & 1611 & 2778 & 1833 \\
\hline 200 & 3900 & 3500 & 4111 & 3889 \\
\hline 210 & 2944 & 1611 & 3611 & 1833 \\
\hline 220 & 1833 & 1667 & 2222 & 2722 \\
\hline 201 & 1111 & 2000 & 1833 & 1889 \\
\hline 211 & 3667 & 3111 & 4167 & 3778 \\
\hline 221 & 1667 & 2444 & 1556 & 2389 \\
\hline 202 & 3111 & 2389 & 3334 & 2500 \\
\hline 212 & 889 & 1889 & 2500 & 2222 \\
\hline 222 & 1444 & 2222 & 1278 & 2222 \\
\hline
\end{tabular}


(continuação)

Grupo 2

\begin{tabular}{|c|c|c|c|c|c|c|c|c|c|c|}
\hline \multirow{2}{*}{$\begin{array}{l}\text { TRATA- } \\
\text { MENTOS }\end{array}$} & \multicolumn{2}{|c|}{ ENSAIO 2.1} & \multicolumn{2}{|c|}{ ENSAID 2.2} & \multicolumn{2}{|c|}{ ENSAIO 2.3} & \multicolumn{2}{|c|}{ ENSAIO 2.4} & \multicolumn{2}{|c|}{ ENSAIO 2.5} \\
\hline & $\begin{array}{l}1 \text { a } \\
\text { Rep. }\end{array}$ & $\begin{array}{c}2 \mathrm{a} \\
\text { Rep. }\end{array}$ & $\begin{array}{r}1 . \\
\text { Rep. }\end{array}$ & $\begin{array}{c}2 \mathrm{a} \\
\text { Rep. }\end{array}$ & $\begin{array}{r}1^{a} \\
\text { Rep. }\end{array}$ & $\begin{array}{c}2^{a} \\
\text { Rep. }\end{array}$ & $\begin{array}{l}1_{a}^{a} \\
\text { Rep. }\end{array}$ & $\begin{array}{r}\text { 2a } \\
\text { Rep. }\end{array}$ & $\begin{array}{l}1: \\
\text { Rep. }\end{array}$ & $\begin{array}{c}2_{0}^{a} \\
\text { Rep. }\end{array}$ \\
\hline 000 & 2113 & 500 & 1556 & 278 & 1084 & 1640 & 336 & 528 & 1667 & 1028 \\
\hline 010 & 1334 & 723 & 1278 & 1111 & 1112 & 1307 & 611 & 444 & 1389 & 1389 \\
\hline 020 & 890 & 778 & 1556 & 1389 & 2919 & 862 & 1056 & 167 & 2278 & 556 \\
\hline 001 & 389 & 1946 & 722 & 1167 & 945 & 2363 & 694 & 1833 & 722 & 2056 \\
\hline 011 & 1112 & & 1778 & 1278 & 1807 & 1.446 & 556 & 1083 & & 1444 \\
\hline 021 & 1001 & 12 & 889 & 1111 & 1307 & 2057 & 1306 & 1056 & & 1712 \\
\hline 002 & 389 & 667 & 778 & 1389 & 1696 & 1334 & 278 & 917 & 1389 & 639 \\
\hline 012 & 890 & 13 & & 722 & 2 & 1529 & 1306 & 11 & & 1222 \\
\hline 022 & 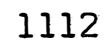 & & & 11. & 1 & 1 & & & & 611 \\
\hline 10 & & & & 100 & & 8 & 1778 & 7 & 2 & 1389 \\
\hline 110 & 2446 & 2446 & 1944 & 1389 & 3558 & 3280 & 1944 & 1917 & & 3056 \\
\hline 120 & 2057 & 2836 & 1556 & 2111 & 3058 & 3253 & 2389 & 2667 & 2472 & 2611 \\
\hline 101 & 1501 & 3169 & 1167 & 1167 & 1918 & 2419 & 1056 & 1944 & 2 & 2222 \\
\hline 111 & 2947 & 2669 & 2222 & 1833 & 2836 & 2419 & 2167 & 1722 & & 2389 \\
\hline 121 & 2057 & 2391 & 1278 & 1944 & 2808 & 3419 & 1139 & 2639 & & 3250 \\
\hline 102 & 1501 & 1612 & 1000 & 1056 & 3253 & 2419 & 1472 & 1556 & 2444 & 1333 \\
\hline 112 & 2280 & 2446 & 1500 & 1556 & 3503 & 2836 & 1556 & 2306 & 2944 & 3194 \\
\hline 122 & 2168 & 2391 & 1222 & 1833 & 3336 & 4392 & 2750 & 2778 & 3222 & 4167 \\
\hline 200 & 3058 & 4003 & 3167 & 3222 & 3197 & 3725 & 2083 & 1667 & 2111 & 3056 \\
\hline 210 & 3670 & 3336 & 3056 & 2389 & 4003 & 3531 & 1583 & 2333 & 4250 & 3139 \\
\hline 220 & 3058 & 3558 & 2667 & 3000 & 2780 & 3836 & 2417 & 3611 & 2723 & 4611 \\
\hline 201 & 3892 & 2724 & 3389 & 2000 & 4281 & 1640 & 1944 & 1000 & 3833 & 1417 \\
\hline 211 & 3725 & 2558 & 3611 & 2111 & 3558 & 5060 & 2222 & 3222 & 3222 & 5000 \\
\hline 221 & 2836 & 2168 & 2667 & 2667 & 3364 & 4087 & 2861 & 2194 & 2611 & 4111 \\
\hline 202 & 1390 & 1501 & 1167 & 1389 & 3253 & 2780 & 2139 & 1889 & 4139 & 3111 \\
\hline 212 & 2669 & 2836 & 2444 & 2667 & 3225 & 3364 & 2389 & 3083 & 3250 & 3472 \\
\hline 222 & 3670 & 2724 & 5166 & 4055 & 4865 & 3197 & 3028 & 3083 & 4528 & 3500 \\
\hline
\end{tabular}


(continuação)

Grupo 3

\begin{tabular}{|c|c|c|c|c|c|c|c|c|c|c|c|c|}
\hline \multirow{2}{*}{$\begin{array}{l}\text { TRATA- } \\
\text { MENTOS }\end{array}$} & \multicolumn{2}{|c|}{ ENSAID 3.1} & \multicolumn{2}{|c|}{ ENSAIO 3.2} & \multicolumn{2}{|c|}{ ENSAIO 3.3} & \multicolumn{2}{|c|}{ ENSAIO 3.4} & \multicolumn{2}{|c|}{ ENSAIO 3.5} & \multicolumn{2}{|c|}{ ENSAIO 3.6} \\
\hline & $\begin{array}{r}1 . \\
\text { Rep. }\end{array}$ & $\begin{array}{r}2 \mathrm{a} \\
\text { Rep. }\end{array}$ & $\begin{array}{l}\text { I. } \\
\text { Rep. }\end{array}$ & $\begin{array}{r}2 . \\
\text { Rep. }\end{array}$ & $\begin{array}{r}1 \text { ? } \\
\text { Rep. }\end{array}$ & $\begin{array}{c}2 \text {. } \\
\text { Rep. }\end{array}$ & $\begin{array}{l}\text { la } \\
\text { Rep. }\end{array}$ & $\begin{array}{r}2 \text { ? } \\
\text { Rep. }\end{array}$ & $\begin{array}{r}\text { I. } \\
\text { Rep. }\end{array}$ & $\begin{array}{r}2^{a} \\
\text { Rep. }\end{array}$ & $\begin{array}{r}1 . \\
\text { Rep. }\end{array}$ & $\begin{array}{r}2^{a} \\
\text { Rep. }\end{array}$ \\
\hline 000 & 834 & 1825 & 1194 & 556 & 733 & 611 & 1807 & 2168 & 2222 & 2417 & 2611 & 2778 \\
\hline 010 & 1056 & 1168 & 722 & 1611 & 278 & 722 & 2446 & 2558 & 2028 & 2306 & 3222 & 2556 \\
\hline 020 & 1446 & 1334 & 1250 & 806 & 567 & 1028 & 2168 & 2141 & 1250 & 3306 & 2556 & 3222 \\
\hline 001 & 1029 & 917 & 667 & 889 & 389 & 667 & 2335 & 2391 & 2556 & 2778 & 2500 & 3139 \\
\hline 011 & 862 & 1863 & 1278 & 556 & 1222 & 800 & 1835 & 2168 & 1278 & 2222 & 3056 & 2611 \\
\hline 021 & 862 & 584 & 1220 & 1583 & 417 & 556 & 1779 & 2280 & 2333 & 2000 & 2778 & 2944 \\
\hline 002 & 1696 & 1334 & 1056 & 1556 & 889 & 1278 & 2502 & 1446 & 2389 & 2694 & 3194 & 2944 \\
\hline 012 & 1807 & 1307 & 917 & 1667 & 511 & 722 & 2224 & 2335 & 2694 & 2444 & 2167 & 3778 \\
\hline 022 & 1001 & 1390 & 1278 & 1450 & 722 & 1222 & 2391 & 2335 & 3194 & 2806 & 2556 & 2778 \\
\hline 100 & 1334 & 1446 & 1472 & 472 & 1889 & 1444 & 2224 & 2168 & 2083 & 3056 & 3861 & 4333 \\
\hline 110 & 2469 & 2530 & 1833 & 2889 & 1861 & 3011 & 2446 & 2502 & 2417 & 2389 & 3556 & 4000 \\
\hline 120 & 1974 & 2085 & 1000 & 2722 & 2000 & 2722 & 2558 & 2335 & 2861 & 2500 & 4056 & 4222 \\
\hline 101 & 1807 & 1918 & 1000 & 1167 & 1278 & 1944 & 1668 & 2280 & 2944 & 2500 & 3722 & 4000 \\
\hline 111 & 1390 & 2613 & 1167 & 2056 & 2083 & 1667 & 862 & 1946 & 2222 & 2028 & 3528 & 2306 \\
\hline 121 & 2186 & 1724 & 1556 & 639 & 2056 & 2528 & 2224 & 1946 & 2861 & 2583 & 4667 & 4000 \\
\hline 102 & 1446 & 1640 & 556 & 2083 & 1694 & 2417 & 2224 & 2113 & 2972 & 2306 & 4722 & 3833 \\
\hline 112 & 2085 & 1473 & 1861 & 2250 & 2222 & 2389 & 2280 & 2558 & 3028 & 2083 & 4778 & 3722 \\
\hline 122 & 1501 & 2335 & 1444 & 2417 & 1733 & 3278 & 1778 & 1557 & 3028 & 2306 & 3889 & 4389 \\
\hline 200 & 2446 & 1307 & 972 & 722 & 1056 & 1667 & 2558 & 2335 & 2139 & 2694 & 4722 & 4000 \\
\hline 210 & 1529 & 2419 & 1450 & 1444 & 2444 & 2889 & 2252 & 2002 & 3056 & 2750 & 5222 & 3833 \\
\hline 220 & 2252 & 1501 & 1111 & 2389 & 2111 & 4056 & 1724 & 2113 & 3056 & 1917 & 4611 & 4056 \\
\hline 201 & 778 & 1779 & 1556 & 1194 & 2444 & 1944 & 1557 & 2335 & 2556 & 2417 & 5000 & 4722 \\
\hline 211 & 2780 & 1696 & 1583 & 2417 & $21 i 1$ & 3389 & 2057 & 2113 & 2944 & 2833 & 5000 & .4567 \\
\hline 221 & 1974 & 2224 & 1611 & 2639 & 3639 & 2778 & 1696 & 2057 & 3056 & 2500 & 5000 & 3917 \\
\hline 204 & 1751 & 2057 & 1611 & 1889 & 2611 & 3167 & 945 & 1501 & 2333 & 1861 & 4667 & 4111 \\
\hline 212 & 1890 & 1835 & 1778 & 2639 & 2972 & 2000 & 1279 & 2168 & 2472 & 2389 & 4278 & 3833 \\
\hline 222 & 1501 & 1501 & 2056 & 2056 & 3278 & 4833 & 1724 & 2513 & 3139 & 2389 & 4444 & 4338 \\
\hline
\end{tabular}


(continuação)

Grupo 4

\begin{tabular}{|c|c|c|c|c|c|c|c|c|}
\hline \multirow[b]{2}{*}{ TRATAMENTOS } & \multicolumn{2}{|c|}{ ENSAIO 4.1} & \multicolumn{2}{|c|}{ ENSAIO 4.2} & \multicolumn{2}{|c|}{ ENSAID 4.3} & \multicolumn{2}{|c|}{ ENSAID 4.4} \\
\hline & $\begin{array}{l}1_{0}^{a} \\
\text { Rep. }\end{array}$ & $\begin{array}{c}2^{a} \\
\text { Rep. }\end{array}$ & $\begin{array}{l}\text { I. } \\
\text { Rep. }\end{array}$ & $\begin{array}{l}2^{a} \\
\text { Rep. }\end{array}$ & $\begin{array}{l}\text { I. } \\
\text { Rep. }\end{array}$ & $\begin{array}{c}2^{a} \\
\text { Rep. }\end{array}$ & $\begin{array}{c}1 . \\
\text { Rep. }\end{array}$ & $\begin{array}{l}2^{a} \\
\text { Rep. }\end{array}$ \\
\hline 000 & 778 & 751 & 222 & 778 & 361 & 2296 & 167 & 1111 \\
\hline 010 & 806 & 306 & 333 & 278 & 2013 & 2335 & 944 & 1500 \\
\hline 020 & 962 & 1234 & 444 & 556 & 2196 & 2013 & 722 & 1389 \\
\hline 001 & 623 & 1329 & 278 & 778 & 901 & 1168 & 667 & 611 \\
\hline 011 & 1585 & 600 & 500 & 556 & 2513 & 2252 & 1444 & 1389 \\
\hline 021 & 695 & 361 & 333 & 500 & 1890 & 2241 & 1000 & 1556 \\
\hline 002 & 945 & 929 & 444 & 556 & 1718 & 306 & 1056 & 333 \\
\hline 012 & 573 & 484 & 333 & 167 & 1557 & 2446 & 944 & 1222 \\
\hline 022 & 684 & 967 & 444 & 167 & 2530 & 2519 & 1389 & 1500 \\
\hline 100 & 1974 & 2180 & 1667 & 667 & 556 & 2363 & 333 & 1111 \\
\hline 110 & 1295 & 2963 & 1278 & 2222 & 2446 & 3325 & 2111 & 1944 \\
\hline 120 & 1462 & 1334 & 1889 & 2111 & 3419 & 2824 & 2389 & 2444 \\
\hline 101 & 917 & 2018 & 556 & 778 & 473 & 906 & 333 & 500 \\
\hline 111 & 1473 & 1112 & 1000 & 1389 & 3319 & 2485 & 1833 & 1556 \\
\hline 121 & 1473 & 1890 & 1889 & 1167 & 3464 & 3030 & 1833 & 2222 \\
\hline 102 & 1140 & 3353 & 889 & 1389 & 1295 & 639 & 667 & 278 \\
\hline 112 & 2430 & 1879 & 1778 & 1111 & 3614 & 3253 & 1889 & 2000 \\
\hline 122 & 1029 & 1279 & 889 & 1278 & 3186 & 3114 & 1889 & 1944 \\
\hline 200 & 1612 & 1640 & 778 & 333 & 111 & 1507 & 278 & 500 \\
\hline 210 & 3486 & 2630 & 1167 & 556 & 1556 & 889 & 3114 & 1084 \\
\hline 220 & 840 & 1407 & 944 & 2833 & 2981 & 3792 & 1667 & 2500 \\
\hline 201 & 2057 & 3408 & 1722 & 1444 & 639 & 456 & 278 & 333 \\
\hline 211 & 4893 & 3597 & 1778 & 1500 & 3075 & 2947 & 1500 & 1222 \\
\hline 221 & 3631 & 3280 & 2278 & 2056 & 4309 & 3964 & 2778 & 2833 \\
\hline 202 & 2341 & 1957 & 556 & 2056 & 2419 & 628 & 1056 & 222 \\
\hline 212 & 3211 & 1473 & 2389 & 1000 & 3870 & 3086 & 1944 & 2222 \\
\hline 222 & 3597 & 3169 & 2722 & 1500 & 4298 & 3975 & 3167 & 2778 \\
\hline
\end{tabular}


(continuação)

Grupo 5

\begin{tabular}{|c|c|c|c|c|c|c|c|c|c|c|}
\hline \multirow{2}{*}{$\begin{array}{l}\text { TRATA- } \\
\text { MENTOS }\end{array}$} & \multicolumn{2}{|c|}{ ENSAIO 5.1} & \multicolumn{2}{|c|}{ ENSAIO 5.2} & \multicolumn{2}{|c|}{ ENSAIO 5.3} & \multicolumn{2}{|c|}{ ENSAIO 5.4} & \multicolumn{2}{|c|}{ ENSAIO 5.5} \\
\hline & $\begin{array}{l}\text { I. } \\
\text { Rep. }\end{array}$ & $\begin{array}{c}2^{a} \\
\text { Rep. }\end{array}$ & $\begin{array}{c}1: \\
\text { Rep. }\end{array}$ & $\begin{array}{c}2^{a} \\
\text { Rep. }\end{array}$ & $\begin{array}{c}1: \\
\text { Rep. }\end{array}$ & $\begin{array}{c}2^{a} \\
\text { Rep. }\end{array}$ & $\begin{array}{c}1_{a}^{a} \\
\text { Rep. }\end{array}$ & $\begin{array}{c}2_{0}^{a} \\
\text { Rep. }\end{array}$ & $\begin{array}{l}\text { 1. } \\
\text { Rep. }\end{array}$ & $\begin{array}{c}2 ! \\
\text { Rep. }\end{array}$ \\
\hline 000 & 1667 & 1444 & 1000 & 889 & 694 & 611 & 667 & 500 & 556 & 1000 \\
\hline 010 & 2278 & 1083 & 1111 & 778 & 1055 & 861 & 917 & 1194 & 889 & 722 \\
\hline 020 & 2056 & 667 & 1278 & 500 & 1111 & 305 & 806 & 1194 & 944 & 900 \\
\hline 001 & 1861 & 1056 & 1687 & 733 & 416 & 749 & 611 & 611 & 611 & 500 \\
\hline 011 & 889 & 1222 & 722 & 1278 & 1027 & 1138 & 778 & 472 & 889 & 389 \\
\hline 021 & 3778 & 1778 & 1111 & 1194 & 2277 & 1055 & 694 & 1472 & 1917 & 556 \\
\hline 002 & 833 & 1972 & 1667 & 1056 & 361 & 749 & 639 & 389 & 444 & 694 \\
\hline 012 & 1444 & 2305 & 1000 & 1677 & 1249 & 1249 & 1361 & 1194 & 1583 & 1389 \\
\hline 022 & 2028 & 1167 & 1000 & 806 & 1388 & 611 & 611 & 833 & 889 & 722 \\
\hline 100 & 3389 & 2278 & 2389 & 2083 & 972 & 1111 & 1500 & 861 & 1500 & 2036 \\
\hline 110 & 3389 & 3556 & 2689 & 2889 & 2055 & 1860 & 2389 & 1611 & 1833 & 2667 \\
\hline 120 & 3445 & 2722 & 2611 & 2056 & 1805 & 2527 & 1833 & 2167 & 2500 & 1556 \\
\hline 101 & 4222 & 3722 & 2500 & 2611 & 583 & 944 & 778 & 806 & 1556 & 1344 \\
\hline 111 & 4139 & 2445 & 3333 & 2356 & 1749 & 1944 & 2167 & 1722 & 2278 & 1333 \\
\hline 121 & 3056 & 2945 & 2556 & 2444 & 1777 & 2138 & 1528 & 2445 & 2222 & 2389 \\
\hline 102 & 3334 & 3611 & 2667 & 2556 & 1527 & 666 & 1056 & 1500 & 2167 & 889 \\
\hline 112 & 4056 & 2667 & 3611 & 2556 & 2277 & 2499 & 2167 & 2861 & 2778 & 2222 \\
\hline 122 & 3417 & 3750 & 2639 & 2333 & 2694 & 2416 & 3185 & 3389 & 3611 & 2778 \\
\hline 200 & 3445 & 3361 & 3722 & 3578 & 694 & 999 & 1111 & 1000 & 1278 & 1917 \\
\hline 210 & 3361 & 3583 & 3778 & 3611 & 2360 & 2083 & 3222 & 2639 & 4167 & 3567 \\
\hline 220 & 2945 & 4222 & 3250 & 3306 & 2110 & 2138 & 2778 & 2861 & 3611 & 3111 \\
\hline 201 & 3056 & 3778 & 667 & 3500 & 444 & 861 & 778 & 250 & 989 & 1528 \\
\hline 211 & 3083 & 2945 & 3722 & 3233 & 1860 & 2083 & 2889 & .2639 & 1528 & 3667 \\
\hline 221 & 4945 & 3167 & 4197 & 3278 & 1666 & 1833 & 1611 & 1750 & 2778 & 2167 \\
\hline 202 & 3778 & 3472 & 3889 & 2833 & 972 & 805 & 1306 & 1833 & 2556 & 2444 \\
\hline 212 & 4111 & 3556 & 3778 & 3778 & 1833 & 1972 & 1833 & 2361 & 3333 & 2667 \\
\hline 222 & 3917 & 3389 & 4278 & 4167 & 2249 & 2333 & 2945 & 3056 & 4833 & 3444 \\
\hline
\end{tabular}


90.

(cont1nuaçäo)

Grupo 6

\begin{tabular}{|c|c|c|c|c|c|c|c|c|c|c|}
\hline \multirow{2}{*}{$\begin{array}{l}\text { TRATA- } \\
\text { MENTOS }\end{array}$} & \multicolumn{2}{|c|}{ ENSAIO 6.1} & \multicolumn{2}{|c|}{ ENSAIO 6.2} & \multicolumn{2}{|c|}{ ENSAIO 6.3} & \multicolumn{2}{|c|}{ ENSAIO 6.4} & \multicolumn{2}{|c|}{ ENSAIO 6.5} \\
\hline & $\begin{array}{l}\text { I. } \\
\text { Rep. }\end{array}$ & $\begin{array}{r}2^{a} \\
\text { Rep. }\end{array}$ & $\begin{array}{l}1: \\
\text { Rep. }\end{array}$ & $\begin{array}{l}2^{\mathrm{a}} \\
\text { Rep. }\end{array}$ & $\begin{array}{r}1 . \\
\text { Rep. }\end{array}$ & $\begin{array}{c}2 . \\
\text { Rep. }\end{array}$ & $\begin{array}{l}\text { 1: } \\
\text { Rep. }\end{array}$ & $\begin{array}{c}2^{a} \\
\text { Rep. }\end{array}$ & $\begin{array}{l}1 . \\
\text { Rep. }\end{array}$ & $\begin{array}{c}2_{0}^{a} \\
R e p .\end{array}$ \\
\hline 000 & 2944 & 2611 & 1942 & 3052 & 1944 & 500 & 722 & 166 & 2717 & 2386 \\
\hline 010 & 2166 & 1999 & 2719 & 1720 & 611 & 500 & 1610 & 777 & 385 & 3441 \\
\hline 020 & 1611 & 1944 & 1165 & 2608 & 1444 & 1278 & 1610 & 1555 & 3474 & 4384 \\
\hline 001 & 2333 & 1388 & 21 & 3552 & 13 & 722 & 944 & 1944 & 998 & 2997 \\
\hline 011 & 1777 & 1722 & 2109 & 2275 & 14 & 556 & 1222 & 1499 & 750 & 3108 \\
\hline 021 & 2777 & 2388 & 2109 & 1831 & 1500 & 1667 & 1166 & 2444 & 3052 & 3274 \\
\hline 002 & 1611 & 1611 & 1276 & 2442 & 944 & 833 & 1055 & 1722 & 2830 & 2497 \\
\hline 0 & 2999 & 1999 & 2275 & 2164 & 944 & 556 & 1055 & 1499 & 2664 & 2886 \\
\hline 022 & 3055 & 2166 & 2109 & 2830 & 778 & 667 & 1722 & 1388 & 3552 & 3052 \\
\hline 100 & 2055 & 1777 & 2331 & 3052 & 2056 & 1000 & 1333 & 944 & 3108 & 2608 \\
\hline 110 & 3722 & 2055 & 2941 & 2997 & 2111 & 1055 & 1888 & 1944 & 4384 & 5439 \\
\hline 120 & 2777 & 1833 & 2442 & 2997 & 1444 & 1167 & 1111 & 2499 & 3108 & 4884 \\
\hline 101 & 4888 & 2222 & 721 & 1998 & 1277 & 1111 & 666 & 888 & 1831 & 1443 \\
\hline 111 & 3499 & 1333 & 1498 & 3274 & 1833 & 556 & 1388 & 1333 & 4051 & 5161 \\
\hline 121 & 2555 & 2111 & 1998 & 2331 & 1278 & 1056 & 1999 & 2110 & 3552 & 4162 \\
\hline 102 & 3055 & 1944 & 2608 & 2442 & 1833 & 1389 & 555 & 1666 & 1332 & 2941 \\
\hline 112 & 2444 & 1833 & 1609 & 2941 & 1167 & 1944 & 2166 & 2333 & 4828 & 3829 \\
\hline 122 & 2555 & 2277 & 2719 & 2664 & 1111 & 1556 & 1722 & 1833 & 4995 & 5439 \\
\hline 200 & 2777 & 1722 & 2053 & 3163 & 1390 & 1111 & 555 & 555 & 3219 & 3889 \\
\hline 210 & 2167 & 2499 & 2830 & 2830 & 1667 & 1611 & 2777 & 2222 & 3219 & 5106 \\
\hline 220 & 3055 & 1611 & 1720 & 2608 & 1278 & 2167 & 722 & 2610 & 3940 & 5994 \\
\hline 201 & 2111 & 1888 & 2941 & 3052 & 1222 & 2056 & 1499 & 555 & 3219 & 2331 \\
\hline 211 & 3555 & 2499 & 2719 & 2941 & 1500 & 833 & 1833 & 2388 & 4551 & 5161 \\
\hline 221 & 2833 & 999 & 2386 & 3385 & 1667 & 1333 & 2053 & 1555 & 4939 & 4162 \\
\hline 202 & 3111 & 1888 & 2608 & 2808 & 1333 & 1333 & 722 & 777 & 2331 & 2941 \\
\hline 212 & 2499 & 1333 & 1665 & 3441 & 1333 & 889 & 1444 & 1808 & 3829 & 4884 \\
\hline 222 & 1833 & 1888 & 2553 & 2331 & 1389 & 2500 & 2166 & 2110 & 5494 & 4773 \\
\hline
\end{tabular}


(contInuação)

Grupo 7

\begin{tabular}{|c|c|c|c|c|}
\hline \multirow[b]{2}{*}{ TRATAMENTOS } & \multicolumn{2}{|c|}{ ENSAIO 7.1} & \multicolumn{2}{|c|}{ ENSAIO 7.2} \\
\hline & $\begin{array}{l}\text { 1. } \\
\text { Rep. }\end{array}$ & $\begin{array}{c}2_{a}^{a} \\
\text { Rep. }\end{array}$ & $\begin{array}{c}\text { la } \\
\text { Rep. }\end{array}$ & $\begin{array}{l}2 \text { a } \\
\text { Rep. }\end{array}$ \\
\hline 000 & 2911 & 2278 & 2944 & 3729 \\
\hline 010 & 2294 & 2733 & 3739 & 2294 \\
\hline 020 & 2139 & 3122 & 2856 & 2956 \\
\hline 001 & 2489 & 2844 & 2406 & 3183 \\
\hline 011 & 2484 & 2483 & 2933 & 2733 \\
\hline 021 & 2383 & 2367 & 2783 & 2589 \\
\hline 002 & 2139 & 1667 & 2767 & 3000 \\
\hline 012 & 3672 & 2422 & 3244 & 3122 \\
\hline 022 & 3411 & 3267 & 2706 & 2556 \\
\hline 100 & 2944 & 3256 & 4028 & 3156 \\
\hline 110 & 2844 & 2194 & 4017 & 3917 \\
\hline 120 & 2667 & 3506 & 3967 & 3661 \\
\hline 101 & 2367 & 2.983 & 3378 & 3967 \\
\hline 111 & 2928 & 2311 & 3478 & 3628 \\
\hline 121 & 3478 & 1778 & 3622 & 3778 \\
\hline 102 & 2644 & 2144 & 2689 & 4000 \\
\hline 112 & 2844 & 2183 & 3589 & 3750 \\
\hline 122 & 3711 & 2778 & 4178 & 3189 \\
\hline 200 & 3633 & 2711 & 4978 & 4222 \\
\hline 210 & 2678 & 3756 & 5217 & 5139 \\
\hline 220 & 1467 & 2906 & 3750 & 5139 \\
\hline 201 & 3250 & 3244 & 3778 & 4411 \\
\hline 211 & 3678 & 2967 & 4178 & 4017 \\
\hline 221 & 2933 & 2567 & 4733 & 4683 \\
\hline 202 & 2322 & 2761 & 4239 & 4194 \\
\hline 212 & 3183 & 2767 & 4850 & 4267 \\
\hline 222 & 3011 & 3011 & 3681 & 4644 \\
\hline
\end{tabular}


(continuação)

Grupo 8

\begin{tabular}{|c|c|c|c|c|c|c|c|c|c|c|}
\hline \multirow{2}{*}{$\begin{array}{l}\text { TRATA- } \\
\text { MENTOS }\end{array}$} & \multicolumn{2}{|c|}{ ENSAIO 8.1} & \multicolumn{2}{|c|}{ ENSAIO 8.2} & \multicolumn{2}{|c|}{ ENSAIO 8.3} & \multicolumn{2}{|c|}{ ENSAIO 8.4} & \multicolumn{2}{|c|}{ ENSAIO 8.5} \\
\hline & $\begin{array}{c}1 . \\
\text { Rep. }\end{array}$ & $\begin{array}{c}2 . \\
\text { rep. }\end{array}$ & $\begin{array}{c}\text { 1. } \\
\text { Rep. }\end{array}$ & $\begin{array}{l}2^{a} \\
\text { Rep. }\end{array}$ & $\begin{array}{l}I^{a} \\
\text { Rep. }\end{array}$ & $\begin{array}{l}2^{a} \cdot \\
\text { Rep. }\end{array}$ & $\begin{array}{l}1 . \\
\text { Rep. }\end{array}$ & $\begin{array}{c}\text { 2. } \\
\text { Rep. }\end{array}$ & $\begin{array}{l}\text { 1. } \\
\text { Rep. }\end{array}$ & $\begin{array}{l}2^{a} . \\
\text { Rep. }\end{array}$ \\
\hline 000 & 2500 & 2500 & 3336 & 2669 & 3056 & 4445 & 5469 & 2868 & 1334 & 000 \\
\hline 010 & 1667 & 2222 & 2502 & 2224 & 4556 & 4167 & 4002 & 3002 & 389 & 4667 \\
\hline 020 & 833 & 2778 & 2280 & 2113 & 4445 & 3611 & 5003 & 4469 & 2500 & 3334 \\
\hline 001 & 1111 & 1945 & 2224 & 2224 & 4723 & 4611 & 4402 & 4135 & 2584 & 3167 \\
\hline 011 & 556 & 2222 & 3336 & 2780 & 4278 & 4167 & 3335 & 4002 & 584 & 361 \\
\hline 021 & 3889 & 3611 & 5115 & 2780 & 4000 & 3056 & 3002 & 4002 & 2395 & 4195 \\
\hline 002 & 2778 & 1945 & 1668 & 2224 & 4445 & 2334 & 2068 & 3802 & 4195 & 1528 \\
\hline 012 & 2500 & 1945 & 3058 & 1390 & 3445 & 3445 & 4602 & 3135 & 3028 & 4361 \\
\hline 02 & 1667 & 1667 & 2669 & 2057 & 4278 & 4723 & 3669 & 2401 & 1500 & 4139 \\
\hline 100 & 444 & 2500 & 3892 & 2780 & 5278 & 3945 & 5069 & 4602 & 4556 & 4639 \\
\hline 110 & 3611 & 4167 & 4615 & 3503 & 3889 & 2778 & 4135 & 3669 & 2732 & 4639 \\
\hline 120 & 3334 & 2222 & 4170 & 3336 & 3778 & 4167 & 4669 & 5069 & 3889 & 3861 \\
\hline 101 & 3334 & 1667 & 4003 & 4170 & 4556 & 2889 & 3468 & 4202 & 2611 & 4584 \\
\hline 111 & 1667 & 3611 & 4615 & 3558 & 4445 & 3000 & 4536 & 4736 & 3806 & 3611 \\
\hline 121 & 2778 & 1211 & 2891 & 3058 & 2889 & 4334 & 3402 & 5069 & 4834 & 5056 \\
\hline 102 & 4445 & 2500 & 2558 & 3781 & 4511 & 4723 & 2001 & 3669 & 1467 & 4306 \\
\hline 112 & 833 & 2222 & 4170 & 3169 & 4334 & 5167 & 3335 & 4536 & 4584 & 4139 \\
\hline 122 & 1945 & 4723 & 4448 & 3336 & 3056 & 4167 & 4469 & 3535 & 2945 & 4611 \\
\hline 200 & 3056 & 3334 & 4114 & 4559 & 4278 & 4445 & 3335 & 2868 & 500 & 3000 \\
\hline 210 & 556 & 1945 & 3114 & 3058 & 4834 & 4223 & 4869 & 4202 & 2223 & 4000 \\
\hline 220 & 2778 & 3334 & 3336 & 2891 & 4167 & 3223 & 1201 & 4336 & 2717 & 4723 \\
\hline 201 & 278 & 2500 & 2502 & 2446 & 2945 & 4334 & 5403 & 4469 & 3417 & 4250 \\
\hline 211 & 1111 & 2778 & 2669 & 3611 & 2889 & 4111 & 4002 & 4135 & 1112 & 3364 \\
\hline 221 & 1111 & 3334 & 4170 & 3336 & 4889 & 2778 & 4002 & 4936 & 1778 & 4473 \\
\hline 202 & 2778 & 4167 & 4448 & 3058 & 4334 & 3334 & 4002 & 3535 & 3417 & 3889 \\
\hline 212 & 1945 & 2500 & 3447 & 4114 & 4723 & 3500 & 3335 & 4602 & 3834 & 4250 \\
\hline 222 & 556 & 4445 & 4281 & 2502 & 4167 & 4167 & 3725 & 5003 & 4111 & 3861 \\
\hline
\end{tabular}


(continuação)

\begin{tabular}{|c|c|c|c|c|c|c|}
\hline \multirow[b]{2}{*}{ TRATAMENTOS } & \multicolumn{2}{|c|}{ ENSAIO 9.1} & \multicolumn{2}{|c|}{ ENSAIO 9.2} & \multicolumn{2}{|c|}{ ENSAIO 9.3} \\
\hline & $\begin{array}{r}1 . \\
\text { Rep. }\end{array}$ & $\begin{array}{l}2^{a} \\
\text { Rep. }\end{array}$ & $\begin{array}{c}1 \mathrm{a} \\
\text { Rep. }\end{array}$ & $\begin{array}{c}2^{a} \\
\text { Rep. }\end{array}$ & $\begin{array}{r}1 . \\
\text { Rep. }\end{array}$ & $\begin{array}{r}\text { 2! } \\
\text { Rep. }\end{array}$ \\
\hline 000 & 334 & 934 & 722 & 1945 & 111 & 556 \\
\hline 010 & 667 & 667 & 1500 & 1500 & 333 & 722 \\
\hline 020 & 467 & 1067 & 1500 & 1111 & 1167 & 333 \\
\hline 001 & 1534 & 1801 & 1778 & 1667 & 2722 & 1222 \\
\hline 011 & 1734 & 2268 & 2056 & 1444 & 2500 & 3167 \\
\hline 021 & 1401 & 734 & 1000 & 1389 & 611 & 389 \\
\hline 002 & 1067 & 1201 & 556 & 1667 & 111 & 1333 \\
\hline 012 & 734 & 667 & 1000 & 1000 & 333 & 556 \\
\hline 022 & 267 & 734 & 833 & 1945 & 278 & 1000 \\
\hline 100 & 1863 & 1801 & 2389 & 2222 & 3389 & 2278 \\
\hline 110 & 1467 & 1601 & 111 & 1667 & 3000 & 3167 \\
\hline 120 & 2935 & 2801 & 1556 & 3556 & 2500 & 3333 \\
\hline 101 & 2935 & 2401 & 2611 & 1778 & 3222 & 2889 \\
\hline 111 & 2268 & 1934 & 1556 & 2611 & 2611 & 3556 \\
\hline 121 & 1668 & 2134 & 1945 & 1945 & 2778 & 2667 \\
\hline 102 & 2268 & 1801 & 1945 & 2056 & 500 & 3389 \\
\hline 112 & 1534 & 2668 & 2111 & 1945 & 2889 & 3333 \\
\hline 122 & 2335 & 2335 & 1389 & 2111 & 2556 & 3722 \\
\hline 200 & 1668 & 2601 & 1389 & 2000 & 3556 & 4167 \\
\hline 210 & 1868 & 2001 & 1667 & 2778 & 4611 & 4389 \\
\hline 220 & 1801 & 1868 & 1945 & 2333 & 3944 & 4388 \\
\hline 201 & 2668 & 2934 & 1945 & 2500 & 6056 & 4111 \\
\hline 211 & 1668 & 2335 & 2000 & 2056 & 3333 & 4333 \\
\hline 221 & 2134 & 2535 & 2500 & 2778 & 4444 & 4444 \\
\hline 202 & 4269 & 2735 & 2500 & 2778 & 3833 & 4556 \\
\hline 212 & 2801 & 1534 & 2222 & 2889 & 4167 & 4667 \\
\hline 222 & 1668 & 3402 & 2111 & 2111 & 4000 & 4278 \\
\hline
\end{tabular}


94.

Tabela 2 - Produção, em kg/ha, referente aos 34 ensaios em blocos casualizados, com a cultura do milho, em diversas regiões do Estado de Pernambuco.

Grupo 1

\begin{tabular}{|c|c|c|c|c|c|c|c|}
\hline \multirow{2}{*}{ ENSAIO } & \multirow{2}{*}{$\begin{array}{l}\text { TRATA- } \\
\text { MENTOS }\end{array}$} & \multicolumn{6}{|c|}{ REPETIC̋̃ES } \\
\hline & & $I$ & II & III & IV & v & VI \\
\hline \multirow{3}{*}{1.1} & $c_{0}$ & 2000 & 2500 & 3333 & 2556 & 611 & 417 \\
\hline & $c_{1}$ & 2833 & 3028 & 2111 & 3333 & 2111 & 972 \\
\hline & $c_{2}$ & 722 & 3194 & 639 & 2417 & 833 & 583 \\
\hline \multirow{3}{*}{1.2} & $c_{0}$ & 2500 & 3222 & 2444 & 2778 & 1667 & 1167 \\
\hline & $c_{1}$ & 2667 & 2611 & 3944 & 2111 & 3778 & 2389 \\
\hline & $c_{2}$ & 1556 & 1667 & 3278 & 1944 & 1500 & 2833 \\
\hline \multirow{3}{*}{1.3} & $c_{0}$ & 2389 & 2500 & 3334 & 1389 & 1667 & 2500 \\
\hline & $c_{1}$ & 4111 & 3222 & 3334 & 4167 & 2667 & 2111 \\
\hline & $c_{2}$ & 2111 & 3889 & 1945 & 3334 & 2778 & 2389 \\
\hline
\end{tabular}


(continuação)

Grupo 2

\begin{tabular}{|c|c|c|c|c|c|c|c|}
\hline \multirow{2}{*}{ ENSAIO } & \multirow{2}{*}{$\begin{array}{l}\text { TRATA- } \\
\text { MENTOS }\end{array}$} & \multicolumn{6}{|c|}{ REPETIÇŐES } \\
\hline & & I & II & III & IV & v & VI \\
\hline \multirow{3}{*}{2.1} & $c_{0}$ & 2558 & 1668 & 2168 & 2280 & 2724 & 2780 \\
\hline & $c_{1}$ & 3503 & 3225 & 3447 & 2502 & 2280 & 2502 \\
\hline & $c_{2}$ & 2780 & 3280 & 2446 & 2502 & 2446 & 2280 \\
\hline \multirow{3}{*}{2.2} & $c_{0}$ & 1667 & 1611 & 1556 & 1722 & 1833 & 1833 \\
\hline & $c_{1}$ & 2611 & 1889 & 2333 & 1278 & 1444 & 1111 \\
\hline & $C_{2}$ & 1944 & 2111 & 1278 & 1778 & 1556 & 1667 \\
\hline \multirow{3}{*}{2.3} & $c_{0}$ & 2944 & 3222 & 4611 & 3000 & 3222 & 2777 \\
\hline & $c_{1}$ & 4055 & 3222 & 3222 & 3777 & 3000 & 1833 \\
\hline & $c_{2}$ & 3777 & 3777 & 3222 & 3777 & 2222 & 2944 \\
\hline \multirow{3}{*}{2.4} & $c_{0}$ & 2975 & 3670 & 2947 & 2446 & 3809 & 2752 \\
\hline & $c_{1}$ & 3531 & 4059 & 2975 & 3141 & 3642 & 2863 \\
\hline & $c_{2}$ & 3169 & 2375 & 3114 & 4726 & 2669 & 2919 \\
\hline \multirow{3}{*}{2.5} & $c_{0}$ & 1250 & 2222 & 1944 & 2861 & 1361 & 1667 \\
\hline & $c_{1}$ & 1528 & 2306 & 1556 & 1389 & 1528 & 1250 \\
\hline & $c_{2}$ & 1722 & 1556 & 1694 & 2833 & 1028 & 2139 \\
\hline \multirow{3}{*}{2.6} & $c_{0}$ & 2778 & 3556 & 2944 & 4056 & 1889 & 2500 \\
\hline & $c_{1}$ & 3000 & 3333 & 2639 & 2944 & 2222 & 2278 \\
\hline & $c_{2}$ & 2528 & 3167 & 3028 & 3944 & 1556 & 3333 \\
\hline
\end{tabular}


(continuação)

Grupo 3

\begin{tabular}{|c|c|c|c|c|c|c|c|}
\hline \multirow{2}{*}{ ENSAIO } & \multirow{2}{*}{$\begin{array}{l}\text { TRATA- } \\
\text { MENTOS }\end{array}$} & \multicolumn{6}{|c|}{ REPETIÇÕES } \\
\hline & & I & II & III & IV & V & $V I$ \\
\hline \multirow{3}{*}{3.1} & $c_{0}$ & 1418 & 2085 & 2029 & 2196 & 2141 & 1446 \\
\hline & $c_{1}$ & 2752 & 1418 & 1946 & 1446 & 2029 & 2057 \\
\hline & $c_{2}$ & 2780 & 2502 & 1974 & 1946 & 1946 & 1446 \\
\hline \multirow{3}{*}{3.2} & $c_{0}$ & 2833 & 1220 & 1333 & 1444 & 1029 & 2000 \\
\hline & $c_{1}$ & 1583 & 1450 & 2056 & 2111 & 1556 & 1851 \\
\hline & $c_{2}$ & 1444 & 1472 & 1250 & 2722 & 1778 & 2611 \\
\hline \multirow{3}{*}{3.3} & $c_{0}$ & 2944 & 2389 & 1333 & 1750 & 1889 & 2167 \\
\hline & $c_{1}$ & 2167 & 2611 & 2556 & 2056 & 2889 & 1889 \\
\hline & $c_{2}$ & 2528 & 2222 & 3000 & 2389 & 2278 & 1911 \\
\hline \multirow{3}{*}{3.4} & $c_{0}$ & 1946 & 1724 & 1168 & 834 & 2307 & 2613 \\
\hline & $c_{1}$ & 2419 & 2069 & 1779 & 2280 & 2113 & 2780 \\
\hline & $c_{2}$ & 2307 & 1279 & 1668 & 1779 & 1557 & 2446 \\
\hline \multirow{3}{*}{3.5} & $c_{0}$ & 3222 & 4778 & 4222 & 4833 & 3722 & 3056 \\
\hline & $c_{1}$ & 3833 & 3833 & 4611 & 3944 & 4250 & 4667 \\
\hline & $c_{2}$ & 3833 & 4889 & 4444 & 4000 & 3889 & 4167 \\
\hline
\end{tabular}


(continuação)

Grupo 4

\begin{tabular}{|c|c|c|c|c|c|c|c|}
\hline \multirow[t]{2}{*}{ ENSAIO } & \multirow{2}{*}{$\begin{array}{l}\text { TRATA- } \\
\text { MENTOS }\end{array}$} & \multicolumn{6}{|c|}{ REPETIÇÕES } \\
\hline & & $I$ & II & III & IV & v & VI \\
\hline \multirow{3}{*}{4.1} & $c_{0}$ & 1918 & 1168 & 2068 & 3002 & 945 & 2402 \\
\hline & $c_{1}$ & 1462 & 2363 & 3097 & 3197 & 1851 & 2891 \\
\hline & $c_{2}$ & 2669 & 1890 & 2263 & 2463 & 2419 & 2196 \\
\hline \multirow{3}{*}{4.2} & $c_{0}$ & 1444 & 944 & 1222 & 1778 & З3з & 1278 \\
\hline & $c_{1}$ & 667 & 1167 & 1778 & 1556 & 944 & 1333 \\
\hline & $c_{2}$ & 1444 & 833 & 1278 & 1167 & 1389 & 667 \\
\hline \multirow{3}{*}{4.3} & $c_{0}$ & 1167 & 1833 & 1389 & 2333 & 1389 & 1667 \\
\hline & $c_{1}$ & 833 & 1667 & 2889 & 2500 & 1389 & 2333 \\
\hline & $\mathrm{C}_{2}$ & 1889 & 1722 & 1778 & 1667 & 2222 & 778 \\
\hline \multirow{3}{*}{4.4} & $c_{0}$ & 1500 & 1667 & 1722 & 2333 & 2000 & 1722 \\
\hline & $c_{1}$ & 1222 & 1667 & 1778 & 2389 & 2167 & 1500 \\
\hline & $c_{2}$ & 1889 & 2000 & 2611 & 1389 & 1944 & 1667 \\
\hline \multirow{3}{*}{4.5} & $c_{0}$ & 3.944 & 1944 & 1389 & 2111 & 1778 & 1778 \\
\hline & $c_{1}$ & 1722 & 1944 & 2167 & 2000 & 2167 & 383 \\
\hline & $c_{2}$ & 222 & 2278 & 1722 & 1500 & 1833 & 1500 \\
\hline
\end{tabular}


(continuação)

\begin{tabular}{|c|c|c|c|c|c|c|c|}
\hline \multirow{2}{*}{ ENSAIO } & \multirow{2}{*}{$\begin{array}{l}\text { TRATA- } \\
\text { MENTOS }\end{array}$} & \multicolumn{6}{|c|}{ REPET IÇÕES } \\
\hline & & I & II & III & IV & v & VI \\
\hline \multirow{3}{*}{5.1} & $c_{0}$ & 3445 & 3824 & 3445 & 3222 & 4056 & 2667 \\
\hline & $c_{1}$ & 3361 & 2695 & 3834 & 2695 & 3695 & 3722 \\
\hline & $c_{2}$ & 3945 & 2667 & 3722 & 3028 & 4167 & 2611 \\
\hline \multirow{3}{*}{5.2} & $c_{0}$ & 1638 & 1416 & 2277 & 1777 & 2694 & 1999 \\
\hline & $c_{1}$ & 1555 & 1972 & 1610 & 1694 & 1972 & 1555 \\
\hline & $c_{2}$ & 1638 & 1944 & 1249 & 1916 & 2499 & 2110 \\
\hline \multirow{3}{*}{5.3} & $c_{0}$ & 1805 & 1556 & 1611 & 2361 & 2417 & 2862 \\
\hline & $c_{1}$ & 2056 & 2500 & 2222 & 2389 & 1500 & 2139 \\
\hline & $c_{2}$ & 2250 & 2083 & 2111 & 2778 & 2000 & 1333 \\
\hline \multirow{3}{*}{5.4} & $c_{0}$ & 2278 & 2500 & 2111 & 1944 & 2667 & 2194 \\
\hline & $c_{1}$ & 2389 & 2389 & 1694 & 3056 & 2389 & 2806 \\
\hline & $c_{2}$ & 2944 & 2444 & 2222 & 2336 & 2889 & 1667 \\
\hline
\end{tabular}


(continuação)

\begin{tabular}{|c|c|c|c|c|c|c|c|}
\hline \multicolumn{8}{|c|}{ Grupo 6} \\
\hline \multirow{2}{*}{ ENSAIO } & \multirow{2}{*}{$\begin{array}{l}\text { TRATA- } \\
\text { MENTOS }\end{array}$} & \multicolumn{6}{|c|}{ REPETIÇÕES } \\
\hline & & I & II & III & IV & V & VI \\
\hline \multirow{3}{*}{6.1} & $c_{0}$ & 2264 & 2886 & 2886 & 1221 & 1720 & 2775 \\
\hline & $c_{1}$ & 3108 & 3330 & 2997 & 2886 & 2442 & 1609 \\
\hline & $c_{2}$ & 2053 & 2640 & 2997 & 3052 & 2664 & 2553 \\
\hline \multirow{3}{*}{6.2} & $c_{0}$ & 1444 & 1389 & 1389 & 1167 & 778 & 500 \\
\hline & $c_{1}$ & 1167 & 1222 & 1333 & 1111 & 1722 & 1111 \\
\hline & $c_{2}$ & 1333 & 722 & 2222 & 1833 & 556 & 1111 \\
\hline \multirow{3}{*}{6.3} & $c_{0}$ & 1499 & 1277 & 2610 & 1722 & 1111 & 1944 \\
\hline & $c_{1}$ & 1388 & 1555 & 2277 & 2055 & 2333 & 1333 \\
\hline & $C_{2}$ & 1666 & 1944 & 1999 & 2333 & 833 & 1222 \\
\hline \multirow{3}{*}{6.4} & $c_{0}$ & 3941 & 3996 & 5217 & 4829 & 4052 & 5439 \\
\hline & $c_{1}$ & 4773 & 3830 & 4718 & 3941 & 5106 & 4329 \\
\hline & $c_{2}$ & 4440 & 4496 & 4829 & 5439 & 4385 & 3608 \\
\hline
\end{tabular}


100.

(continuação)

Grupo 8

\begin{tabular}{|c|c|c|c|c|c|c|c|}
\hline \multirow{2}{*}{ ENSAIO } & \multirow{2}{*}{$\begin{array}{l}\text { TRATA- } \\
\text { MENTOS }\end{array}$} & \multicolumn{6}{|c|}{ REPETIÇס̋ES } \\
\hline & & I & II & III & IV & v & VI \\
\hline \multirow{3}{*}{8.1} & $c_{0}$ & 3611 & 1111 & 1945 & 1945 & 2778 & 4167 \\
\hline & $c_{1}$ & 4445 & 2778 & 556 & 2500 & 4167 & 3056 \\
\hline & $c_{2}$ & 3611 & 1945 & 833 & 1945 & 2778 & 2778 \\
\hline \multirow{3}{*}{8.2} & $c_{0}$ & 4170 & 4448 & 3114 & 3002 & 3558 & 3781 \\
\hline & $c_{1}$ & 3392 & 3169 & 3058 & 2780 & 3781 & 3948 \\
\hline & $c_{2}$ & 5282 & 4170 & 3558 & 3447 & 4170 & 3892 \\
\hline \multirow{3}{*}{8.3} & $c_{0}$ & 3334 & 4723 & 4167 & 4611 & 4278 & 3334 \\
\hline & $c_{1}$ & 4445 & 5556 & 3889 & 5278 & 5000 & 3611 \\
\hline & $\mathrm{c}_{2}$ & 3111 & 5723 & 3889 & 5000 & 3611 & 4445 \\
\hline \multirow{3}{*}{8.4} & $c_{0}$ & 4469 & 4469 & 4936 & 4469 & 3869 & 4002 \\
\hline & $c_{1}$ & 4002 & 4202 & 3135 & 3869 & 4402 & 4536 \\
\hline & $c_{2}$ & 3335 & 4869 & 4669 & 4668 & 3335 & 4669 \\
\hline \multirow{3}{*}{8.5} & $c_{0}$ & 2277 & 3694 & 2084 & 4973 & 3917 & 4445 \\
\hline & $c_{1}$ & 2223 & 2945 & 3750 & 3611 & 4667 & 4195 \\
\hline & $c_{2}$ & 3473 & 3084 & 2834 & 4139 & 4556 & 4667 \\
\hline
\end{tabular}


(continuaçāo)

\section{Grupo 9}

\begin{tabular}{cccccccc}
\hline \multirow{2}{*}{ ENSAIO } & $\begin{array}{c}\text { TRATA- } \\
\text { MENTOS }\end{array}$ & $I$ & II & III & IV & $V$ & VI \\
\hline \multirow{4}{*}{9.1} & $C_{0}$ & 3202 & 1868 & 1668 & 1668 & 1734 & 2001 \\
& $C_{1}$ & 1668 & 2268 & 1934 & 1801 & 1801 & 2801 \\
& $C_{2}$ & 2935 & 2268 & 2735 & 1868 & 3669 & 2068 \\
& & & & & & & \\
& $C_{0} .2$ & 3056 & 2500 & 3111 & 2500 & 2444 & 3333 \\
& $C_{1}$ & 2778 & 2167 & 3333 & 2889 & 3611 & 3444 \\
& $C_{2}$ & 3111 & 2778 & 3111 & 3667 & 1222 & 4167 \\
& & & & & & & \\
\hline
\end{tabular}


Tabela 3 - Desvios padrão, em kg/ha e coeficientes de variação $(\%)$, dos ensalos individuals e dos grupos fatoriais.

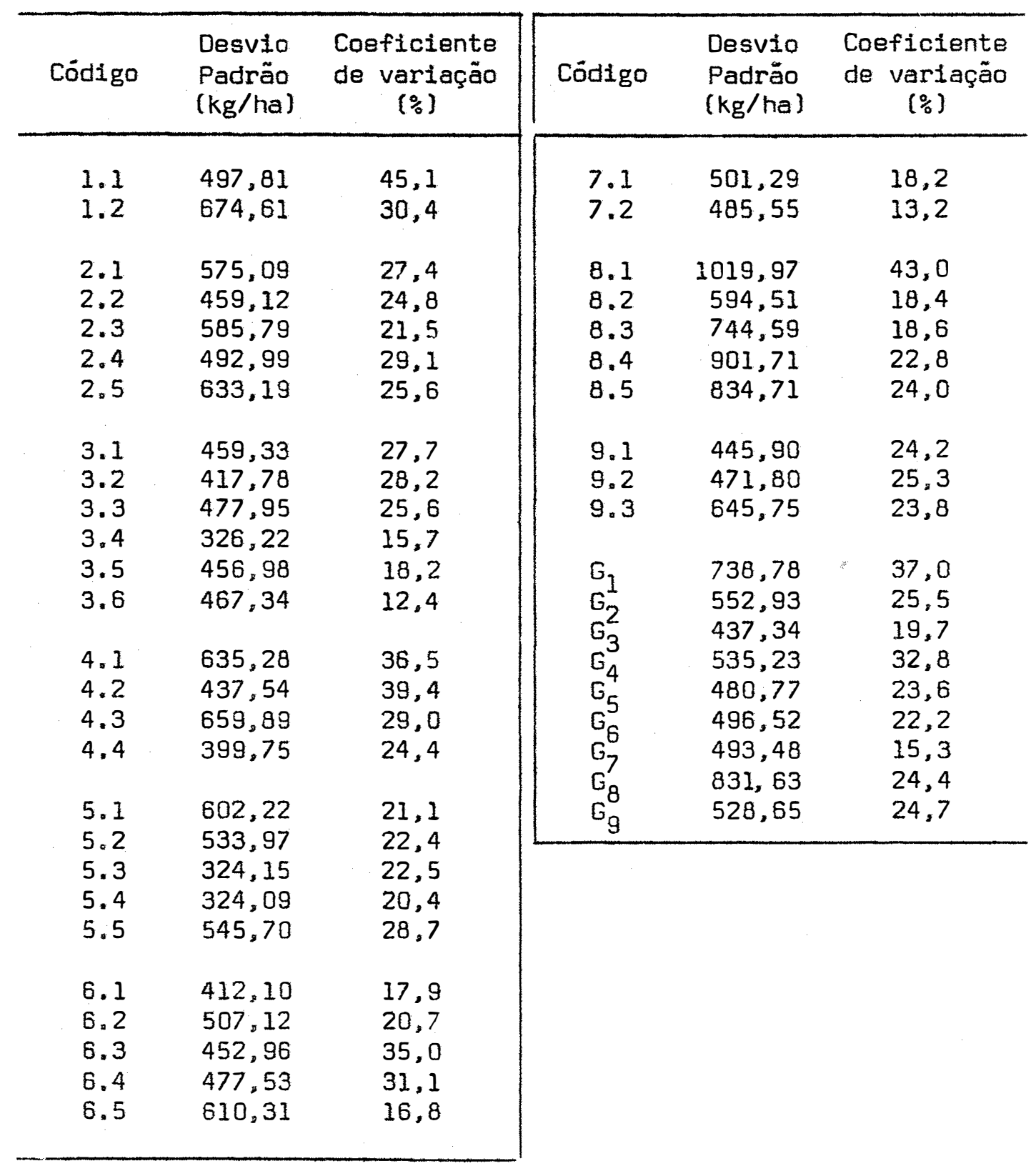


Tabela 4 - Desvios padrão, em $\mathrm{kg} /$ ha e coeficientes de variação $(\%)$, dos 34 ensaios individuais e dos grupos de ensaios em blo cos casualizados.

\begin{tabular}{|c|c|c|}
\hline Cödigo & $\begin{array}{l}\text { Desvio } \\
\text { Padrão } \\
\text { (kg/ha) }\end{array}$ & $\begin{array}{c}\text { Coeficiente } \\
\text { de variação } \\
(\%)\end{array}$ \\
\hline $\begin{array}{l}1.1 \\
1.2 \\
1.3\end{array}$ & $\begin{array}{l}715,79 \\
781,90 \\
815,51\end{array}$ & $\begin{array}{l}37,7 \\
31,9 \\
29,5\end{array}$ \\
\hline $\begin{array}{l}2.1 \\
2.2 \\
2.3 \\
2.4 \\
2.5 \\
2.6\end{array}$ & $\begin{array}{l}492,93 \\
411,46 \\
615,33 \\
637,38 \\
467,26 \\
411,47\end{array}$ & $\begin{array}{l}18,7 \\
22,3 \\
18,9 \\
19,7 \\
26,4 \\
14,3\end{array}$ \\
\hline $\begin{array}{l}3.1 \\
3.2 \\
3.3 \\
3.4 \\
3.5\end{array}$ & $\begin{array}{l}471,83 \\
535,93 \\
477,16 \\
400,77 \\
528,93\end{array}$ & $\begin{array}{l}23,9 \\
30,4 \\
21,0 \\
20,2 \\
12,8\end{array}$ \\
\hline $\begin{array}{l}4.1 \\
4.2 \\
4.3 \\
4.4 \\
4.5\end{array}$ & $\begin{array}{l}521,26 \\
404,48 \\
595,98 \\
377,60 \\
565,73\end{array}$ & $\begin{array}{l}23,3 \\
34,3 \\
34,1 \\
20,5 \\
33,5\end{array}$ \\
\hline $\begin{array}{l}5.1 \\
5.2 \\
5.3 \\
5.4\end{array}$ & $\begin{array}{l}466,08 \\
322,41 \\
495,34 \\
415,98\end{array}$ & $\begin{array}{l}13,8 \\
17,3 \\
23,5 \\
17,5\end{array}$ \\
\hline $\begin{array}{l}6.1 \\
6.2 \\
6.3 \\
6.4\end{array}$ & $\begin{array}{l}584,75 \\
446,00 \\
461,43 \\
639,81\end{array}$ & $\begin{array}{l}22,8 \\
36,3 \\
26,7 \\
14,1\end{array}$ \\
\hline
\end{tabular}

\begin{tabular}{|ccc}
\hline Código & $\begin{array}{c}\text { Desvio } \\
\text { Padrão } \\
(\mathrm{kg} / \mathrm{ha})\end{array}$ & $\begin{array}{c}\text { Coeficiente } \\
\text { de variação } \\
(\%)\end{array}$ \\
\hline 8.1 & 664,57 & 25,5 \\
8.2 & 410,26 & 11,1 \\
8.3 & 506,71 & 11,7 \\
8.4 & 596,51 & 14,1 \\
8.5 & 623,26 & 17,1 \\
& & \\
9.1 & 604,67 & 27,2 \\
9.2 & 639,79 & 21,6 \\
& & 32,5 \\
$G_{1}$ & 772,18 & 19,9 \\
$G_{2}^{1}$ & 513,94 & 20,0 \\
$G_{3}^{2}$ & 485,37 & 28,8 \\
$G_{4}^{3}$ & 500,63 & 17,7 \\
$G_{5}^{4}$ & 429,99 & 21,5 \\
$G_{6}^{5}$ & 539,24 & 15,3 \\
$G_{8}^{6}$ & 567,63 & 24,1 \\
$G_{9}$ & 622,48 & \\
\hline
\end{tabular}


Tabela 5 - Análise conjunta dos 9 grupos de ensaios fatoriais.

\begin{tabular}{lrrrr}
\multicolumn{5}{c}{ Grupo I } \\
\hline Fontes de Variação & G.L. & S.Q. & Q.M. & F \\
\hline Ensaios $(E)$ & 1 & 5.461 .652 & & \\
Nitrogēnio (N) & 2 & 13.241 .279 & 6.620 .639 & $20,65 \%$ \\
Interação (EXN) & 2 & 641.347 & 320.673 & 0,59 \\
Resíduo Mëdio & 48 & 26.198 .517 & 545.802 & \\
\hline $\bar{N}_{0}=1.686 \mathrm{~kg} / \mathrm{ha}$ & $\bar{N}_{1}=1.815 \mathrm{~kg} / \mathrm{ha}$ & $\bar{N}_{2}=2.485 \mathrm{~kg} / \mathrm{ha}$
\end{tabular}

\begin{tabular}{lcrrr}
\hline Fontes de Variação & G.L. & \multicolumn{1}{c}{ S.Q. } & Q.M. & $F$ \\
\hline Ensaios $(E)$ & 1 & 5.461 .652 & & \\
Fósforo $(P)$ & 2 & 4.797 .475 & 2.398 .737 & 7.32 \\
Interação (ExP) & 2 & 655.355 & 327.677 & 0.60 \\
Resíduo Mëdio & 48 & 26.198 .517 & 545.802 & \\
\hline $\bar{P}_{0}=2.014 \mathrm{~kg} / \mathrm{ha}$ & $\bar{P}_{1}=2.243 \mathrm{~kg} / \mathrm{ha}$ & $\bar{P}_{2}=1.728 \mathrm{~kg} / \mathrm{ha}$
\end{tabular}

\begin{tabular}{lcccc}
\hline Fontes de Variação & G.L. & S.Q. & Q.M. & $F$ \\
\hline Ensaios $(E)$ & 1 & 5.461 .652 & & \\
Potässio $(K)$ & 2 & 3.827 .407 & 1.913 .703 & 13,37 \\
Interação $(E x K)$ & 2 & 286.303 & 143.151 & 0,26 \\
Resíduo Mëdio & 48 & 26.198 .517 & 545.802 & \\
\hline $\bar{K}_{0}=2.168 \mathrm{~kg} / \mathrm{ha}$ & $\bar{K}_{1}=1.733 \mathrm{~kg} / \mathrm{ha}$ & $\bar{K}_{2}=2.085 \mathrm{~kg} / \mathrm{ha}$ \\
$*$ & & &
\end{tabular}


(continuação)

Grupo 2

\begin{tabular}{lrrrr}
\hline Fontes de Variação & G.L. & S.Q. & Q.M. & $F$ \\
\hline Ensaios $(E)$ & 4 & 39.275 .333 & & \\
Nitrogênio $(N)$ & 2 & 158.641 .620 & 79.320 .810 & $78,91 * *$ \\
Interação $(E \times N)$ & 8 & 8.042 .076 & 1.005 .259 & $3,29 * *$ \\
Resíduo Mëdio & 120 & 36.687 .459 & 305.729 & \\
\hline $\bar{N}_{0}=1.175 \mathrm{~kg} / \mathrm{ha}$ & $\bar{N}_{1}=2.282 \mathrm{~kg} / \mathrm{ha}$ & $\bar{N}_{2}=3.109 \mathrm{~kg} / \mathrm{ha}$
\end{tabular}

\begin{tabular}{lrrrr}
\hline Fontes de Variação & G.L. & S.Q. & $0 . M$. & $F$ \\
\hline Ensaios $(E)$ & 4 & 39.275 .333 & & \\
Fósforo $(P)$ & 2 & 13.138 .435 & 6.569 .217 & 31.31 ** \\
Interação (ExP) & 8 & 1.678 .688 & 209.836 & 0.69 \\
Residuo Médio & 120 & 36.687 .459 & 305.729 & \\
\hline $\bar{P}_{0}=1.860 \mathrm{~kg} / \mathrm{ha}$ & $\bar{P}_{1}=2.268 \mathrm{~kg} / \mathrm{ha}$ & $\bar{P}_{2}=2.371 \mathrm{~kg} / \mathrm{ha}$
\end{tabular}

\begin{tabular}{lrrrr}
\hline Fontes de Variação & G.L. & \multicolumn{1}{c}{ S.Q. } & Q.M. & \multicolumn{1}{l}{$F$} \\
\hline Ensaios $(E)$ & 4 & 39.275 .333 & & \\
Potássio $(\mathrm{K})$ & 2 & 178.801 & 89.401 & 0.23 \\
Interação (ExK) & 8 & 3.121 .855 & 390.232 & 1.28 \\
Residuo Mëdio & 120 & 36.687 .459 & 305.729 & \\
\hline$\overline{\mathrm{K}}_{0}=2.195 \mathrm{~kg} / \mathrm{ha}$ & $\overline{\mathrm{K}}_{1}=2.171 \mathrm{~kg} / \mathrm{ha}$ & $\overline{\mathrm{K}}_{2}=2.133 \mathrm{~kg} / \mathrm{ha}$
\end{tabular}


(continuação)

Desdobramento dos graus de liberdade de nitrogênio:

\begin{tabular}{lcrrrr}
\hline Fontes de Variação & G.L. & \multicolumn{1}{c}{ S.Q. } & Q.M. & \multicolumn{1}{c}{$F$} \\
\hline Ensaios (E) & 4 & 39.275 .333 & & \\
Nitrogênio Linear (N') & 1 & 156.837 .335 & 156.837 .335 & $285.53^{* \%}$ \\
Interação (ExN') & 4 & 2.197 .128 & 549.282 & \\
\hline
\end{tabular}

\begin{tabular}{lrrrrl}
\hline Fontes de Variação & G.L. & S.Q. & Q.M. & F \\
\hline Ensaios (E) & 4 & 39.275 .333 & & \\
Nitrogênio Quadrät. $\left(N^{\prime \prime}\right)$ & 1 & 1.804 .285 & 1.804 .285 & 1.23 \\
Interação (ExN") & 4 & 5.844 .948 & 1.461 .237 & \\
\hline
\end{tabular}

Desdobramento dos graus de liberdade de fósforo:

\begin{tabular}{lccrcr}
\hline Fontes de Variação & G.L. & S.Q. & Q.M. & F \\
\hline Ensaios (E) & 4 & 39.275 .333 & & \\
Fósforo Linear ( $P^{\prime}$ ) & 1 & 11.734 .609 & 11.734 .609 & $35.11^{* \% *}$ \\
Interação (ExP') & 4 & 1.336 .875 & 334.219 & \\
\hline
\end{tabular}

\begin{tabular}{lrrrr}
\hline Fontes de Variação & G.L. & \multicolumn{1}{c}{ S.Q. } & Q.M. & \multicolumn{1}{c}{$F$} \\
\hline Ensaios (E) & 4 & 39.275 .333 & & \\
Fósforo Quadrático(P") & 1 & 1.403 .826 & 1.403 .826 & $16.43^{* * *}$ \\
Interação (ExP") & 4 & 341.813 & 85.453 & \\
\hline
\end{tabular}


(continuação)

Grupo 3

\begin{tabular}{lrrrr}
\hline Fontes de Variação & G.L. & S.Q. & Q.M. & $F$ \\
\hline Ensaios (E) & 5 & 187.767 .324 & & \\
N1trogën1o $(N)$ & 2 & 37.615 .356 & 18.807 .678 & $55.40 \% *$ \\
Interação (EXN) & 10 & 33.951 .110 & 339.511 & 1.77 \\
Residugo Méd1o & 144 & 27.542 .869 & 191.270 & \\
\hline $\bar{N}_{0}=1.755 \mathrm{~kg} / \mathrm{ha}$ & $\bar{N}_{1}=2.372 \mathrm{~kg} / \mathrm{ha}$ & $\bar{N}_{2}=2.551 \mathrm{~kg} / \mathrm{ha}$
\end{tabular}

\begin{tabular}{lrrrr}
\hline Fontes de Variação & G.L. & \multicolumn{1}{c}{ S.Q. } & Q.M. & F \\
\hline Ensaios $(E)$ & 5 & 187.767 .324 & & \\
Fösforo $(P)$ & 2 & 2.961 .374 & 1.480 .687 & 2,82 \\
Interação $($ ExP) & 10 & 5.242 .627 & 524.263 & $2,74 \% *$ \\
Resfduo Mëdio & 144 & 27.542 .869 & 191.270 & \\
\hline $\bar{P}_{0}=2.096 \mathrm{~kg} / \mathrm{ha}$ & $\bar{P}_{1}=2.257 \mathrm{~kg} / \mathrm{ha}$ & $\bar{P}_{2}=2.324 \mathrm{~kg} / \mathrm{ha}$
\end{tabular}

\begin{tabular}{lrrrl}
\hline Fontes de Variação & G.L. & S.Q. & Q.M. & $F$ \\
\hline Ensaios $(E)$ & 5 & 187.767 .324 & & \\
Potässio $(K)$ & 2 & 1.151 .136 & 575.568 & 2.04 \\
Interação $($ ExK) & 10 & 2.823 .052 & 282.305 & 1.48 \\
Residuo Mëdio & 144 & 27.542 .869 & 191.270 & \\
\hline $\bar{K}_{0}=2.214 . \mathrm{kg} / \mathrm{ha}$ & $\bar{K}_{I}=2.160 \mathrm{~kg} / \mathrm{ha}$ & $\bar{K}_{2}=2.304 \mathrm{~kg} / \mathrm{ha}$
\end{tabular}


(continuação)

Desdobramento dos graus de liberdade do nitrogênio:

\begin{tabular}{lccccc}
\hline Fontes de Variação & G.L. & S.Q. & Q.M. & F \\
\hline Ensaios (E) & 5 & 187.767 .324 & & \\
Nitrogênio Linear (N') & 1 & 34.162 .748 & 34.162 .748 & 5.23 \\
Interação (EXN') & 5 & 32.634 .627 & 6.526 .925 & \\
\hline
\end{tabular}

\begin{tabular}{lrrrr}
\hline Fontes de Variação & G.L. & \multicolumn{1}{c}{ S.Q. } & Q.M. & \multicolumn{1}{c}{$F$} \\
\hline Ensaios (E) & 5 & 187.767 .324 & & \\
Nitrogênio Quadrät, $\left(N^{\prime \prime}\right)$ & 1 & 3.452 .608 & 3.452 .608 & $13.11 * *$ \\
Interação (ExN") & 5 & 1.316 .483 & 263.297 & \\
\hline
\end{tabular}


(continuação)

Grupo 4

\begin{tabular}{lccrr}
\hline Fontes de Variação & G.L. & S.Q. & Q.M. & \multicolumn{1}{c}{$F$} \\
\hline Ensaios $(E)$ & 3 & 40.959 .187 & & \\
Nitrogênio $(N)$ & 2 & 42.961 .588 & 21.480 .794 & $12.88 * *$ \\
Interação $(E x N)$ & 6 & 10.003 .988 & 1.667 .331 & $5.82 \% *$ \\
Resíduo Médio & 96 & 27.501 .645 & 286.475 & \\
\hline $\bar{N}_{0}=1.034 \mathrm{~kg} / \mathrm{ha}$ & $\bar{N}_{1}=1.752 \mathrm{~kg} / \mathrm{ha}$ & $\bar{N}_{2}=2.107 \mathrm{~kg} / \mathrm{ha}$
\end{tabular}

\begin{tabular}{lccccc}
\hline Fontes de Variação & G.L. & S.Q. & Q.M. & $F$ \\
\hline Ensaios $(E)$ & 3 & 40.959 .187 & & \\
Fósforo $(P)$ & 2 & 39.573 .631 & 19.786 .815 & 4.46 \\
Interação (ExP) & 6 & 26.606 .485 & 4.434 .414 & $15.48 * *$ \\
Residuo Médio & 96 & 27.501 .645 & 286.475 & \\
\hline $\bar{P}_{0}=1.034 \mathrm{~kg} / \mathrm{ha}$ & $\bar{P}_{1}=1.845 \mathrm{~kg} / \mathrm{ha}$ & $\bar{P}_{2}=2.014 \mathrm{~kg} / \mathrm{ha}$
\end{tabular}

\begin{tabular}{lcrrl}
\hline Fontes de Variação & G.L. & \multicolumn{1}{c}{ S.Q. } & M. & $F$ \\
\hline Ensaios $(E)$ & 3 & 40.959 .187 & & \\
Potássio $(K)$ & 2 & 888.032 & 444.016 & 1.54 \\
Interação $($ ExK) & 6 & 1.726 .187 & 287.698 & 1.00 \\
Resíduo Médio & 96 & 27.501 .645 & 286.475 & \\
\hline $\bar{K}_{0}=1.542 \mathrm{~kg} / \mathrm{ha}$ & $\bar{K}_{1}=1.658 \mathrm{~kg} / \mathrm{ha}$ & $\overline{\mathrm{K}}_{2}=1.692 \mathrm{~kg} / \mathrm{ha}$
\end{tabular}


(continuação)

Desdobramento dos graus de liberdade do nitrogênio:

\begin{tabular}{lcrrrr}
\hline Fontes de Variação & G.L. & \multicolumn{1}{c}{ S.Q. } & Q.M. & $F$ \\
\hline Ensaios (E) & 3 & 40.959 .187 & & \\
Nitrogênio Linear (N') & 1 & 42.961 .588 & 42.961 .588 & $13,91^{\text {म }}$ \\
Interação (ExN') & 3 & 9.264 .803 & 3.088 .268 & \\
\hline
\end{tabular}

\begin{tabular}{lrrrrr}
\hline Fontes de Variação & G.L. & \multicolumn{1}{c}{ S.Q. } & \multicolumn{1}{c}{ Q.M. } & F \\
\hline Ensaios (E) & 3 & 40.959 .187 & & \\
Nitrogênio Quadrät. (N") & 1 & 1.579 .172 & 1.579 .172 & 8.41 \\
Interação (ExN") & 3 & 739.185 & 246.395 & \\
\hline
\end{tabular}


(continuação)

\begin{tabular}{|c|c|c|c|c|}
\hline & & Grupo 5 & & \\
\hline Fontes de Variação & G.L. & $S . Q$. & Q.M. & $F$ \\
\hline Ensaios (E) & 4 & 73.202 .991 & & \\
\hline Nitrogênio $(N)$ & 2 & 130.720 .689 & 65.360 .344 & $29,58^{* \%}$ \\
\hline Interação (ExN) & 8 & 17.677 .915 & 2.209 .739 & $9,56^{* * *}$ \\
\hline Resíduo Médio & 120 & 27.736 .583 & 231.138 & \\
\hline
\end{tabular}

\begin{tabular}{|c|c|c|c|c|}
\hline Fontes de Variação & G.L. & $S \cdot Q$. & Q.M. & $\mathrm{F}$ \\
\hline Ensaios (E) & 4 & 73.202 .991 & & \\
\hline Fósforo $(P)$ & 2 & 24.694 .592 & 12.347 .296 & $9,81 \%$ \\
\hline Interação (ExP) & 8 & 10.071 .670 & 1.258 .959 & $5,45 \% *$ \\
\hline Resíduo Médio & 120 & 27.736 .583 & 231.138 & \\
\hline
\end{tabular}

\begin{tabular}{lrrrr}
\hline Fontes de Variação & G.L. & S.Q. & Q.M. & $F$ \\
\hline Ensaios $(E)$ & 4 & 73.202 .991 & & \\
Potássio $(K)$ & 2 & 4.696 .353 & 2.348 .177 & $8.00^{*}$ \\
Interação $($ ExK) & 8 & 2.348 .247 & 293.531 & 1.27 \\
Resĩduo Médio & 120 & 27.736 .583 & 231.138 & \\
\hline $\bar{K}_{0}=1.997 \mathrm{~kg} / \mathrm{ha}$ & $\bar{K}_{1}=1.893 \mathrm{~kg} / \mathrm{ha}$ & $\overline{\mathrm{K}}_{2}=2.210 \mathrm{~kg} / \mathrm{ha}$
\end{tabular}


(continuação)

Desdobramento dos graus de liberdade do nitrogênio:

\begin{tabular}{lcrrrr}
\hline Fontes de Variação & G.L. & \multicolumn{1}{c}{ S.Q. } & Q.M. & F \\
\hline Ensaios (E) & 4 & 73.202 .991 & & \\
Nitrogênio Linear (N') & $l$ & 118.410 .867 & 118.410 .867 & $29,35^{* *}$ \\
Interação (ExN') & 4 & 16.139 .384 & 4.034 .846 & \\
\hline
\end{tabular}

\begin{tabular}{lcrrrr}
\hline Fontes de Variação & G.L. & S.Q. & Q.M. & $F$ \\
\hline Ensaios (E) & 4 & 73.202 .991 & & \\
Nitrogênio Quadrät. (N") & 1 & 12.309 .822 & 12.309 .822 & $32.00^{* *}$ \\
Interação (ExN") & 4 & 1.538 .531 & 384.633 & \\
\hline
\end{tabular}

Desdobramento dos graus de liberdade do fósforo:

\begin{tabular}{lcrrr}
\hline Fontes de Variação & G.L. & S.Q. & Q.M. & F \\
\hline Ensaios (E) & 4 & 73.202 .991 & & \\
Fósforo Linear ( $\left.P^{\circ}\right)$ & 1 & 20.179 .066 & 20.179 .066 & $10.68^{*}$ \\
Interação (ExP') & 4 & 7.556 .236 & 1.889 .059 & \\
\hline
\end{tabular}

\begin{tabular}{lcrrrr}
\hline Fontes de Variação & G.L. & S.Q. & Q.M. & F \\
\hline Ensaios (E) & 4 & 73.202 .991 & & \\
Fósforo Quadrático(P") & 1 & 4.515 .527 & 4.515 .527 & 7.18 \\
Interação (ExP") & 4 & 2.515 .434 & 628.858 & \\
\hline
\end{tabular}


(continuação)

Desdobramento dos graus de liberdade do potássio:

\begin{tabular}{lcrrr}
\hline Fontes de Variação & G.L. & \multicolumn{1}{c}{ S.Q. } & \multicolumn{1}{c}{ Q.M. } & \multicolumn{1}{c}{$F$} \\
\hline Ensaios (E) & 4 & 73.202 .991 & & \\
Potássio Linear (K') & $I$ & 2.034 .369 & 2.034 .369 & $79.13^{* *}$ \\
Interação (ExK') & 4 & 102.834 & 25.708 & \\
\hline
\end{tabular}

\begin{tabular}{lrrrrr}
\hline Fontes de Variação & G.L. & \multicolumn{1}{c}{ S.Q. } & Q.M. & F \\
\hline Ensaios (E) & 4 & 73.202 .991 & & \\
Potássio Quadrático(K") & 1 & 2.661 .984 & 2.661 .984 & 4.74 \\
Interação (ExK") & 4 & 2.245 .413 & 561.353 & \\
\hline
\end{tabular}


(continuação)

\section{Grupo 6}

\begin{tabular}{lrrrr}
\hline Fontes de Variação & G.L. & S.Q. & Q.M. & $F$ \\
\hline Ensaios $(E)$ & 4 & 181.560 .769 & & \\
Nitrogénio $(N)$ & 2 & 11.017 .503 & 5.508 .751 & $7.94 \%$ \\
Interação $(E \times N)$ & 8 & 5.548 .633 & 693.579 & $2,81^{* * *}$ \\
Resíduo Médio & 120 & 29.584 .416 & 246.537 & \\
$\overline{\bar{N}}_{0}=1.962 \mathrm{~kg} / \mathrm{ha}$ & $\bar{N}_{1}=2.321 \mathrm{~kg} / \mathrm{ha}$ & $\bar{N}_{2}=2.436 \mathrm{~kg} / \mathrm{ha}$
\end{tabular}

\begin{tabular}{lrrrc}
\hline Fontes de Variação & G.L. & S.Q. & Q.M. & $F$ \\
\hline Ensaios $(E)$ & 4 & 181.560 .769 & & \\
Fósforo $(P)$ & 2 & 11.510 .333 & 5.755 .166 & 1.88 \\
Interação $(E x P)$ & 8 & 24.479 .628 & 3.059 .953 & $12.41 \% \%$ \\
Residuo Médio & 120 & 29.584 .416 & 246.537 & \\
\hline $\bar{P}_{0}=1.950 \mathrm{~kg} / \mathrm{ha}$ & $\bar{P}_{1}=2.356 \mathrm{~kg} / \mathrm{ha}$ & $\bar{P}_{2}=2.414 \mathrm{~kg} / \mathrm{ha}$
\end{tabular}

\begin{tabular}{lrrrl}
\hline Fontes de Variação & G.L. & \multicolumn{1}{c}{ S.Q. } & Q.M. & F \\
\hline Ensaios $(E)$ & 4 & 181.560 .769 & & \\
Potássio $(K)$ & 2 & 569.563 & 284.781 & 1.72 \\
Interação (ExK) & 8 & 1.326 .534 & 165.817 & 0.67 \\
Residuo Médio & 120 & 29.584 .416 & 246.537 & \\
\hline $\bar{K}_{0}=2.305 \mathrm{~kg} / \mathrm{ha}$ & $\bar{K}_{1}=2.204 \mathrm{~kg} / \mathrm{ha}$ & $\overline{\mathrm{K}}_{2}=2.210 \mathrm{~kg} / \mathrm{ha}$
\end{tabular}


(continuação)

Desdobramento dos graus de liberdade do nitrogênio:

\begin{tabular}{lcrrr}
\hline Fontes de Variação & G.L. & \multicolumn{1}{c}{ S.Q. } & \multicolumn{1}{c}{ Q.M. } & F \\
\hline Ensalos (E) & 4 & 181.560 .769 & & \\
Nitrogênio Linear (N') & 1 & 10.119 .902 & 10.119 .902 & $8.34^{* *}$ \\
Interação (ExN') & 4 & 4.854 .020 & 1.213 .505 & \\
\hline
\end{tabular}

\begin{tabular}{lrrrr}
\hline Fontes de Variação & G.L. & \multicolumn{1}{c}{ S.Q. } & Q.M. & F \\
\hline Ensaios (E) & 4 & 181.560 .769 & & \\
N1trogênio Quadrāt.(N") & 1 & 897.600 & 897.600 & 5.17 \\
Interação (ExN") & 4 & 694.613 & 173.653 & \\
\hline
\end{tabular}


(continuação)

Grupo 7

\begin{tabular}{|c|c|c|c|c|}
\hline Fontes de Variação & G.L. & $S . Q$. & Q.M. & $F$ \\
\hline Ensaios & 1 & 23.270 .532 & & \\
\hline Nitrogēnio $(\mathrm{N})$ & 2 & 15.448 .585 & 7.724 .292 & 2,33 \\
\hline Interação (ExN) & 2 & 6.628 .293 & 3.314 .147 & $13,61 * *$ \\
\hline Resíduo Médio & 48 & 11.689 .286 & 243.527 & \\
\hline
\end{tabular}

\begin{tabular}{lrrrl}
\hline Fontes de Variação & G.L. & \multicolumn{1}{c}{ S.Q. } & \multicolumn{1}{l}{ Q.M. } & F \\
\hline Ensalos $(E)$ & 1 & 23.270 .532 & & \\
Fósforo $(P)$ & 2 & 353.338 & 176.669 & 8,00 \\
Interação (ExP) & 2 & 44.139 & 22.069 & 0,09 \\
Resíduo Médio & 48 & 11.689 .286 & 243.527 & \\
\hline $\bar{P}_{0}=3.157 \mathrm{~kg} / \mathrm{ha}$ & $\bar{P}_{1}=3.293 \mathrm{~kg} / \mathrm{ha}$ & $\bar{P}_{2}=3.193 \mathrm{~kg} / \mathrm{ha}$
\end{tabular}

\begin{tabular}{|c|c|c|c|c|}
\hline Fontes de Variação & G.L. & $S . Q$. & Q.M. & $F$ \\
\hline Ensaios (E) & 1 & 23.270 .532 & & \\
\hline Potässio (K) & 2 & 723.804 & 361.902 & 1,89 \\
\hline Interação (ExK) & 2 & 382.279 & 191.139 & 0,78 \\
\hline Residuo Médio & 48 & 11.689 .286 & 243.527 & \\
\hline
\end{tabular}


(continuação)

Grupo 8

\begin{tabular}{lrrrr}
\hline Fontes de Variação & G.L. & S.Q. & Q.M. & $F$ \\
\hline Ensaios (E) & 4 & 95.306 .267 & & \\
Nitrogênio (N) & 2 & 12.124 .879 & 6.062 .439 & $5.70^{*}$ \\
Interação (ExN) & 8 & 8.512 .413 & 1.064 .052 & 1.54 \\
Resíduo Médio & 120 & 82.993 .178 & 691.610 & \\
\hline $\bar{N}_{0}=3.149 \mathrm{~kg} / \mathrm{ha}$ & $\bar{N}_{1}=3.608 \mathrm{~kg} / \mathrm{ha}$ & $\bar{N}_{2}=3.404 \mathrm{~kg} / \mathrm{ha}$
\end{tabular}

\begin{tabular}{lrrrr}
\hline Fontes de Variação & G.L. & \multicolumn{1}{c}{ S.Q. } & Q.M. & $F$ \\
\hline Ensaios $(E)$ & 4 & 95.306 .267 & & \\
Fósforo $(P)$ & 2 & 484.830 & 242.415 & 0.50 \\
Interação (ExP) & 8 & 3.886 .417 & 485.802 & 0.70 \\
Resíduo Médio & 120 & 82.993 .178 & 691.610 & \\
\hline $\bar{P}_{0}=3.360 \mathrm{~kg} / \mathrm{ha}$ & $\bar{P}_{1}=3.398 \mathrm{~kg} / \mathrm{ha}$ & $\bar{P}_{2}=3.463 \mathrm{~kg} / \mathrm{ha}$
\end{tabular}

\begin{tabular}{lrrrr}
\hline Fontes de Variação & G.L. & \multicolumn{1}{c}{ S.Q. } & Q.M. & $F$ \\
\hline Ensaios $(E)$ & 4 & 95.306 .267 & & \\
Potássio $(K)$ & 2 & 41.787 & 20.893 & 0.03 \\
Interação (EXK) & 8 & 6.151 .890 & 768.986 & 1.11 \\
Resíduo Médio & 120 & 82.993 .178 & 691.610 & \\
$\overline{\bar{K}}_{0}=3.423 \mathrm{~kg} / \mathrm{ha}$ & $\overline{\mathrm{K}}_{1}=3.405 \mathrm{~kg} / \mathrm{ha}$ & $\overline{\mathrm{K}}_{2}=3.393 \mathrm{~kg} / \mathrm{ha}$
\end{tabular}


(continuação)

Desdobramento dos graus de liberdade do nitrogênio:

\begin{tabular}{lccccc}
\hline Fontes de Variação & G.L. & S.Q. & Q.M. & F \\
\hline Ensaios (E) & 4 & 95.306 .267 & & \\
Nitrogênio Linear(N') & 1 & 2.934 .546 & 2.934 .546 & 2.63 \\
Interação (ExN') & 4 & 4.459 .996 & 1.114 .999 & \\
\hline
\end{tabular}

\begin{tabular}{lccccc}
\hline Fontes de Variação & G.L. & S.Q. & Q.M. & F \\
\hline Ensaios (E) & 4 & 95.306 .267 & & \\
Nitrogênio Quadrát. (N") & 1 & 9.190 .333 & 9.190 .333 & $9.07 *$ \\
Interação (ExN") & 4 & 4.052 .417 & 1.013 .104 & \\
\hline
\end{tabular}


(continuação)

Grupo 9

\begin{tabular}{lcccc}
\hline Fontes de Variação & G.L. & S.Q. & Q.M. & $F$ \\
\hline Ensaios (E) & 2 & 26.627 .672 & & \\
Nitrogênio (N) & 2 & 95.525 .138 & 47.762 .569 & 6.19 \\
Interação (ExN) & 4 & 30.851 .903 & 7.712 .976 & $27.60 \% \%$ \\
Resíduo Médio & 72 & 20.121 .973 & 279.472 & \\
\hline $\bar{N}_{0}=1.117 \mathrm{~kg} / \mathrm{ha}$ & $\bar{N}_{1}=2.334 \mathrm{~kg} / \mathrm{ha}$ & $\bar{N}_{2}=2.968 \mathrm{~kg} / \mathrm{ha}$
\end{tabular}

\begin{tabular}{lrrrr}
\hline Fontes de Variação & G.L. & \multicolumn{1}{c}{ S.Q. } & Q.M. & F \\
\hline Ensaios (E) & 2 & 26.627 .672 & & \\
Fösforo $(P)$ & 2 & 393.758 & 196.879 & 0.46 \\
Interação (ExP) & 4 & 1.690 .069 & 422.517 & 1.51 \\
Residuo Médio & 72 & 20.121 .973 & 279.472 & \\
\hline$\overline{P_{0}}=2.208 \mathrm{~kg} / \mathrm{ha}$ & $\bar{P}_{1}=2.115 \mathrm{~kg} / \mathrm{ha}$ & $\bar{P}_{2}=2.096 \mathrm{~kg} / \mathrm{ha}$
\end{tabular}

\begin{tabular}{|c|c|c|c|c|}
\hline Fontes de Variação & G.L. & $S . Q$. & Q.M. & $F$ \\
\hline Ensaios (E) & 2 & 26.627 .672 & & \\
\hline Potássio (K) & 2 & 4.488 .268 & 2.244 .134 & $7,02 *$ \\
\hline Interação (ExK) & 4 & 1.278 .435 & 319.809 & 1,14 \\
\hline Resíduo Médio & 72 & 20.121 .973 & 279.472 & \\
\hline
\end{tabular}


Tabela 6 - Análise conjunta dos 8 grupos de ensaios em blocos casua lizados.

\begin{tabular}{|c|c|c|c|c|}
\hline \multicolumn{5}{|c|}{ Grupo 1} \\
\hline Fontes de Variação & G.L. & $S . Q$. & Q.M. & $\mathrm{F}$ \\
\hline Ensaios (E) & 2 & 6.952 .472 & & \\
\hline Calcário (C) & 2 & 6.511 .709 & 3.255 .855 & $9,34 \%$ \\
\hline Interação (ExC) & 4 & 1.394 .546 & 348.637 & 0,58 \\
\hline Resíduo Médio & 30 & 17.887 .733 & 596.258 & \\
\hline $\bar{c}_{0}=2.165 \mathrm{~kg} / \mathrm{ha}$ & $\overline{c_{1}}$ & $\begin{array}{l}861 \mathrm{~kg} / \mathrm{ha} \\
\text { upo } 2\end{array}$ & $\overline{\mathrm{c}}_{2}=$ & $89 \mathrm{~kg} / \mathrm{ha}$ \\
\hline Fontes de Variação & G.L. & $S \cdot Q$. & Q.M. & $\mathrm{F}$ \\
\hline Ensaios (E) & 5 & 42.441 .239 & & \\
\hline Calcārio (C) & 2 & 66.400 & 33.200 & 0,21 \\
\hline Interação (ExC) & 10 & 1.570 .256 & 157.026 & 0,59 \\
\hline Resíduo Médio & 60 & 15.847 .962 & 264.133 & \\
\hline $\bar{c}_{o}=2.550 \mathrm{~kg} / \mathrm{ha}$ & $\bar{\Gamma}$ & $\begin{array}{l}595 \mathrm{~kg} / \mathrm{ha} \\
\text { upo } 3\end{array}$ & $\overline{c_{2}}=$ & $08 \mathrm{~kg} / \mathrm{ha}$ \\
\hline Fontes de Variação & G.L. & $S . Q$. & Q.M. & $F$ \\
\hline Ensaios (E) & 4 & 67.280 .593 & & \\
\hline Calcário $(\mathrm{C})$ & 2 & 1.097 .674 & 548.837 & $4,65^{*}$ \\
\hline Interação (ExC) & 8 & 944.370 & 118.046 & 0,50 \\
\hline Resíduo Médio & 50 & 11.779 .160 & 235.583 & \\
\hline
\end{tabular}


(continuação)

Desdobramento dos graus de liberdade do calcărio no Grupo 3:

\begin{tabular}{lcrrrr}
\hline Fontes de Variação & G.L. & \multicolumn{1}{c}{ S.Q. } & \multicolumn{1}{c}{ Q.M. } & \multicolumn{1}{c}{$F$} \\
\hline Ensaios (E) & 4 & 67.280 .593 & & \\
Calcärio Linear (C') & 1 & 679.683 & 679.683 & $30,73^{* *}$ \\
Interação (ExC') & 4 & 88.463 & 22.116 & \\
\hline
\end{tabular}

\begin{tabular}{lcccc}
\hline Fontes de Variação & G.L. & S.Q. & Q.M. & F \\
\hline Ensaios (E) & 4 & 67.280 .593 & & \\
Calcário Quadrático(C") & 1 & 417.990 & 417.990 & 1.95 \\
Interação (ExC") & 4 & 855.907 & 213.977 & \\
\hline
\end{tabular}

Grupo 4

\begin{tabular}{|c|c|c|c|c|}
\hline Fontes de Variação & G.L. & $S . Q$. & Q.M. & $F$ \\
\hline Ensaios (E) & 4 & 10.347 .655 & & \\
\hline Calcário (C) & 2 & 431.929 & 215.965 & 1,33 \\
\hline Interação (ExC) & 8 & 1.296 .446 & 162.056 & 0,64 \\
\hline Resíduo Médio & 50 & 12.531 .505 & 250.630 & \\
\hline $\bar{C}_{0}=1.672 \mathrm{~kg} / \mathrm{ha}$ & & $\begin{array}{l}834 \mathrm{~kg} / \mathrm{ha} \\
\text { upo } 5\end{array}$ & $\bar{C}_{2}=$ & $10 \mathrm{~kg} / \mathrm{h}$ \\
\hline Fontes de Variação & G.L. & $S \cdot Q=$ & Q.M. & $F$ \\
\hline Ensaios (E) & 3 & 23.882 .815 & & \\
\hline Calcário (C) & 2 & 17.705 & 8.853 & 0,17 \\
\hline Interação $(E \times C)$ & 6 & 309.016 & 51.503 & 0,28 \\
\hline Resíduo Médio & 40 & 7.395 .786 & 184.895 & \\
\hline
\end{tabular}


(continuação)

Grupo 6

\begin{tabular}{lrrrr}
\hline Fontes de Varjação & G.L. & \multicolumn{1}{c}{ S.Q. } & Q.M. & F \\
\hline Ensaios $(E)$ & 3 & 113.379 .392 & & \\
Calcário $(C)$ & 2 & 304.455 & 152.227 & 1.48 \\
Interação $(E \times C)$ & 6 & 617.915 & 102.986 & 0.35 \\
Resíduo Médio & 40 & 11.631 .288 & 290.782 & \\
\hline $\bar{C}_{0}=2.419 \mathrm{~kg} / \mathrm{ha}$ & $\overline{\mathrm{C}}_{1}=2.570 \mathrm{~kg} / \mathrm{ha}$ & $\overline{\mathrm{C}}_{2}=2.539 \mathrm{~kg} / \mathrm{ha}$
\end{tabular}

Grupo 8

\begin{tabular}{|c|c|c|c|c|}
\hline Fontes de Variação & G.L. & $S . Q$. & Q.M. & $F$ \\
\hline Ensaios (E) & 4 & 33.554 .757 & & \\
\hline Calcário (C) & 2 & 131.650 & 65.825 & 0,13 \\
\hline Interação (ExC) & 8 & 4.086 .538 & 510.817 & 1,58 \\
\hline Residuo Médio & 50 & 16.110 .063 & 322.201 & \\
\hline$\overline{\mathrm{c}}_{\mathrm{o}}=3.656 \mathrm{~kg} / \mathrm{ha}$ & \multicolumn{2}{|c|}{$\bar{c}_{1}=3.698 \mathrm{~kg} / \mathrm{ha}$} & \multicolumn{2}{|c|}{$\bar{c}_{2}=3.750 \mathrm{~kg} / \mathrm{hc}$} \\
\hline Fontes de Variação & G.L. & s.o. & Q.M. & $\mathrm{F}$ \\
\hline Ensaios (E) & 1 & 4.887 .784 & & \\
\hline Calcário (C) & 2 & 888.864 & 444.432 & 1,74 \\
\hline Interação $(E \times C)$ & 2 & 510.098 & 255.049 & 0,66 \\
\hline Resíduo Médio & 20 & 7.749 .548 & 387.477 & \\
\hline
\end{tabular}


123.

Tabela 7 - Estimativas dos parâmetros e respectivos intervalos de confiança, ao nível de $95 \%$ de probabilidade, das equações de superfícies de resposta para os ensaios individuals em esquema fatorial.

\begin{tabular}{|c|c|c|c|c|c|c|c|c|c|c|}
\hline Cödi & $\bar{a}_{0}$ & $\hat{a}_{11}$ & $\hat{a}_{22}$ & $\hat{a}_{33}$ & $\hat{a}_{12}$ & $\bar{a}_{13}$ & $\hat{a}_{23}$ & $\hat{a}_{14}$ & $\hat{a}_{24}$ & $\hat{a}_{34}$ \\
\hline & 1,8 & 3 & & & & 87,1 & -25 & 210,1 & & -3 \\
\hline 1.1 & $\begin{array}{l}1466,2 \\
2040,6\end{array}$ & $\begin{array}{l}168,4 \\
631,1\end{array}$ & $\begin{array}{r}-213,6 \\
249,1\end{array}$ & $\begin{array}{l}501,7 \\
964,4\end{array}$ & $\begin{array}{r}-136,2 \\
190,9\end{array}$ & $\begin{array}{r}-160,6 \\
166,5\end{array}$ & $\begin{array}{r}68,3 \\
395,4\end{array}$ & $\begin{array}{l}473,4 \\
736,7\end{array}$ & $\begin{array}{r}-116,2 \\
147,1\end{array}$ & $\begin{array}{l}-37,1 \\
226,2\end{array}$ \\
\hline 1.2 & $\begin{array}{l}1651,3 \\
2137,0 \\
2622,7\end{array}$ & $\begin{array}{l}-19,5 \\
371,8 \\
763,1\end{array}$ & $\begin{array}{l}-922,1 \\
-530,8 \\
-139,5\end{array}$ & $\begin{array}{r}-107,5 \\
283,8 \\
675,1\end{array}$ & $\begin{array}{r}-392,3 \\
-115,7 \\
160,9\end{array}$ & $\begin{array}{r}-533,4 \\
-256,8 \\
19,8\end{array}$ & $\begin{array}{r}-302,1 \\
-25,5 \\
251,1\end{array}$ & $\begin{array}{l}103,0 \\
325,6 \\
548,2\end{array}$ & $\begin{array}{r}-392,4 \\
-169,8 \\
52,8\end{array}$ & $\begin{array}{l}-268,9 \\
-46,3 \\
176,3\end{array}$ \\
\hline 2. & $\begin{array}{l}2155,1 \\
2569,2 \\
2983,3\end{array}$ & $\begin{array}{r}-622,1 \\
-288,5 \\
45,1\end{array}$ & $\begin{array}{r}-594,4 \\
-260,8 \\
72,8\end{array}$ & $\begin{array}{r}-493,3 \\
-159,7 \\
173,9\end{array}$ & $\begin{array}{r}-175,1 \\
60,7 \\
296,5\end{array}$ & $\begin{array}{r}-437,3 \\
-201,5 \\
34,3\end{array}$ & $\begin{array}{r}42,3 \\
278,1 \\
513,9\end{array}$ & $\begin{array}{r}775,2 \\
965,0 \\
1154,8\end{array}$ & $\begin{array}{r}-98,9 \\
90,9 \\
280,7\end{array}$ & $\begin{array}{r}-429,2 \\
-239,4 \\
-49,6\end{array}$ \\
\hline .2 & $\begin{array}{l}1585,3 \\
1915,8\end{array}$ & $\begin{array}{l}167,4 \\
433,7 \\
700,0\end{array}$ & $\begin{array}{r}-327,9 \\
-61,6 \\
204,7\end{array}$ & $\begin{array}{r}-244,7 \\
21,6 \\
287,9\end{array}$ & $\begin{array}{l}-19,2 \\
169,0 \\
357,2\end{array}$ & $\begin{array}{r}-197,5 \\
-9,3 \\
178,9\end{array}$ & & $\begin{array}{l}680,2 \\
831,7 \\
983,2\end{array}$ & $\begin{array}{l}126,2 \\
277,7 \\
429,2\end{array}$ & $\begin{array}{r}-238,0 \\
-86,5 \\
65,0\end{array}$ \\
\hline .3 & $\begin{array}{l}2611,7 \\
3033,5 \\
3455,3\end{array}$ & $\begin{array}{l}-782,3 \\
-442,5 \\
-102,7\end{array}$ & $\begin{array}{r}-46 \\
-12 \\
20\end{array}$ & $\begin{array}{r}-240,3 \\
99,5 \\
439,3\end{array}$ & $\begin{array}{r}-161,5 \\
78,7 \\
318,9\end{array}$ & $\begin{array}{r}-299,2 \\
-59,0 \\
181,2\end{array}$ & $\begin{array}{r}-123,3 \\
116,9 \\
357,1\end{array}$ & $\begin{array}{l}7,4 \\
0,7 \\
7,0\end{array}$ & $\begin{array}{r}66,2 \\
259,5 \\
452,8\end{array}$ & $\begin{array}{r}-146,1 \\
47,2 \\
240,5\end{array}$ \\
\hline 2.4 & $\begin{array}{l}1641,3 \\
1996,3 \\
2351,2\end{array}$ & $\begin{array}{r}-634,0 \\
-348,1 \\
-62,2\end{array}$ & $\begin{array}{r}-379,1 \\
-93,2 \\
192,7\end{array}$ & $\begin{array}{r}-295,7 \\
-9,8 \\
276,1\end{array}$ & $\begin{array}{r}78,0 \\
280,1 \\
482,2\end{array}$ & $\begin{array}{r}-176,5 \\
25,6 \\
227,7\end{array}$ & $\begin{array}{r}-237,8 \\
-35,8 \\
166,3\end{array}$ & $\begin{array}{l}632,7 \\
795,4 \\
958,1\end{array}$ & $\begin{array}{l}166,7 \\
329,4 \\
492,1\end{array}$ & $\begin{array}{l}-50,9 \\
111,8 \\
274,5\end{array}$ \\
\hline 2 & $\begin{array}{l}2303,6 \\
2759,5 \\
3215,4\end{array}$ & $\begin{array}{r}-588,9 \\
-221,7 \\
145,5\end{array}$ & $\begin{array}{r}-586,5 \\
-219,3 \\
147,9\end{array}$ & $\begin{array}{r}-354,3 \\
12,9 \\
380,1\end{array}$ & $\begin{array}{l}-45,0 \\
214,6 \\
474,2\end{array}$ & & $\begin{array}{r}-282,7 \\
-23,1 \\
236,5\end{array}$ & $\begin{array}{r}895,4 \\
1104,4 \\
1313,4\end{array}$ & $\begin{array}{l}110,2 \\
319,2 \\
528,2\end{array}$ & $\begin{array}{r}-198,4 \\
10,6 \\
219,6\end{array}$ \\
\hline 3.1 & $\begin{array}{l}1672,3 \\
2003,0 \\
2333,7\end{array}$ & $\begin{array}{r}-610,2 \\
-343,8 \\
-77,4\end{array}$ & $\begin{array}{l}-21,3 \\
245,1 \\
511,5\end{array}$ & $\begin{array}{r}-196,0 \\
70,4 \\
336,8\end{array}$ & $\begin{array}{r}-141,1 \\
47,2 \\
235,5\end{array}$ & $\begin{array}{r}-263,0 \\
-74,7 \\
113,6\end{array}$ & $\begin{array}{r}-275,6 \\
-87,3 \\
101,0\end{array}$ & $\begin{array}{l}151,3 \\
302,9 \\
454,5\end{array}$ & $\begin{array}{r}-95,2 \\
56,4 \\
208,0\end{array}$ & $\begin{array}{r}-190,6 \\
-39,0 \\
112,6\end{array}$ \\
\hline 3.2 & $\begin{array}{l}1373,6 \\
1674,4 \\
1975,2\end{array}$ & $\begin{array}{r}-401,8 \\
-159,5 \\
82,8\end{array}$ & $\begin{array}{r}-529,6 \\
-287,3 \\
-45,0\end{array}$ & $\begin{array}{l}-84,6 \\
157,7 \\
400,0\end{array}$ & $\begin{array}{r}-79,8 \\
91,5 \\
262,8\end{array}$ & $\begin{array}{r}-79,2 \\
92,1 \\
263,4\end{array}$ & $\begin{array}{r}-229,4 \\
-58,1 \\
113,2\end{array}$ & $\begin{array}{l}162,3 \\
300,2 \\
438,1\end{array}$ & $\begin{array}{l}102,9 \\
240,8 \\
378,7\end{array}$ & $\begin{array}{r}25,8 \\
163,7 \\
301,6\end{array}$ \\
\hline 3.3 & $\begin{array}{l}1663,0 \\
2007,1 \\
2351,2\end{array}$ & $\begin{array}{r}-658,0 \\
-380,8 \\
103,6\end{array}$ & $\begin{array}{r}-247,8 \\
29,4 \\
306,6\end{array}$ & $\begin{array}{r}-132,7 \\
144,5 \\
421,7\end{array}$ & $\begin{array}{l}131,6 \\
327,5 \\
523,4\end{array}$ & $\begin{array}{l}-61,2 \\
134,7 \\
330,6\end{array}$ & $\begin{array}{r}-282,3 \\
-86,4 \\
109,5\end{array}$ & $\begin{array}{r}843,8 \\
1001,5 \\
1159,2\end{array}$ & $\begin{array}{l}159,0 \\
316,7 \\
474,4\end{array}$ & $\begin{array}{r}32,6 \\
190,3 \\
348,0\end{array}$ \\
\hline
\end{tabular}


124.

(continuação)

\begin{tabular}{|c|c|c|c|c|c|c|c|c|c|c|}
\hline ödigo & $\hat{a}_{0}$ & $\tilde{a}_{11}$ & $\hat{a}_{22}$ & $\hat{a}_{33}$ & $\hat{a}_{12}$ & $\hat{a}_{13}$ & $\hat{a}_{23}$ & $\hat{a}_{14}$ & $\hat{a}_{24}$ & $\hat{a}_{34}$ \\
\hline 3.4 & $\begin{array}{l}99,8 \\
34,7 \\
69,6\end{array}$ & $\begin{array}{r}-219,7 \\
-30,5 \\
158,7\end{array}$ & $\begin{array}{r}-249,9 \\
-60,7 \\
128,5\end{array}$ & $\begin{array}{l}-41,4 \\
147,8 \\
337,0\end{array}$ & $\begin{array}{r}-127,4 \\
6,3 \\
140,0\end{array}$ & $\begin{array}{r}-250,3 \\
-116,6 \\
17,1\end{array}$ & $\begin{array}{r}-59,2 \\
74,5 \\
208,2\end{array}$ & $\begin{array}{r}-229,3 \\
-121,7 \\
-14,1\end{array}$ & $\begin{array}{r}-86,4 \\
21,2 \\
128,8\end{array}$ & $\begin{array}{r}-236,3 \\
-128,7 \\
-21,2\end{array}$ \\
\hline 35 & $\begin{array}{l}2113,8 \\
2442,8 \\
2771,8\end{array}$ & $\begin{array}{r}-346,1 \\
-81,0 \\
184,1\end{array}$ & $\begin{array}{r}-130,8 \\
134,3 \\
399,4\end{array}$ & $\begin{array}{r}-216,7 \\
48,7 \\
313,8\end{array}$ & $\begin{array}{r}-94,7 \\
92,7 \\
280,1\end{array}$ & $\begin{array}{r}-342,4 \\
-155,0 \\
32,4\end{array}$ & $\begin{array}{r}-102,8 \\
84,5 \\
271,9\end{array}$ & $\begin{array}{r}-51,3 \\
99,5 \\
250,9\end{array}$ & $\begin{array}{r}-90,6 \\
60,2 \\
211,0\end{array}$ & $\begin{array}{r}-93,0 \\
57,8 \\
208,6\end{array}$ \\
\hline 3 & $\begin{array}{l}3525,9 \\
3862,4 \\
4198,9\end{array}$ & $\begin{array}{r}-589,3 \\
-318,3 \\
-47,3\end{array}$ & $\begin{array}{r}-125,0 \\
146,0 \\
417,0\end{array}$ & $\begin{array}{r}-245,3 \\
25,7 \\
296,7\end{array}$ & $\begin{array}{r}-213,4 \\
-21,8 \\
169,8\end{array}$ & $\begin{array}{r}-243,5 \\
-51,9 \\
139,7\end{array}$ & $\begin{array}{l}-253,9 \\
-62,3 \\
129,3\end{array}$ & & $\begin{array}{l}-166,3 \\
-12,1 \\
142,1\end{array}$ & $\begin{array}{r}27,9 \\
182,1\end{array}$ \\
\hline & $\begin{array}{l}1667,3 \\
2124,7 \\
2582,1\end{array}$ & $\begin{array}{r}-356,3 \\
12,2 \\
380,7\end{array}$ & $\begin{array}{r}-655,9 \\
-287,4 \\
81,1\end{array}$ & $\begin{array}{r}-668,0 \\
-299,5 \\
69,0\end{array}$ & $\begin{array}{r}-120,5 \\
140,0 \\
400,5\end{array}$ & $\begin{array}{l}-77,7 \\
182,8 \\
443,3\end{array}$ & $\begin{array}{r}-187,3 \\
73,2 \\
333,7\end{array}$ & $\begin{array}{r}72 \\
93 \\
114\end{array}$ & $\begin{array}{l}-227,9 \\
-18,3 \\
191,3\end{array}$ & $\begin{array}{r}-104,6 \\
105,0 \\
314,6\end{array}$ \\
\hline & $\begin{array}{l}1048,4 \\
1363,4 \\
1678,4\end{array}$ & $\begin{array}{r}-584,1 \\
-330,4 \\
-76,7\end{array}$ & $\begin{array}{r}-259,9 \\
-6,2 \\
247,5\end{array}$ & $\begin{array}{r}-297,0 \\
-43,3 \\
210,4\end{array}$ & $\begin{array}{r}72,9 \\
252,3 \\
431,7\end{array}$ & $\begin{array}{l}-38,1 \\
141,3 \\
320,7\end{array}$ & $\begin{array}{r}-313,7 \\
-134,3 \\
45,1\end{array}$ & & $\begin{array}{r}80,9 \\
225,3 \\
369,7\end{array}$ & $\begin{array}{r}-147,5 \\
-3,1 \\
141,3\end{array}$ \\
\hline & $\begin{array}{l}2317,2 \\
2792,4 \\
3267,6\end{array}$ & $\begin{array}{r}-608,1 \\
-225,3 \\
157,5\end{array}$ & $\begin{array}{l}-1010,1 \\
-627,3 \\
-244,5\end{array}$ & $\begin{array}{r}-301,5 \\
81,3 \\
464,1\end{array}$ & $\begin{array}{l}184,4 \\
455,0 \\
725,6\end{array}$ & & $\begin{array}{r}-162,9 \\
107,7 \\
378,3\end{array}$ & & $\begin{array}{r}810,1 \\
1027,9 \\
1245,7\end{array}$ & $\begin{array}{l}153,3 \\
371,1\end{array}$ \\
\hline & $\begin{array}{l}1446,5 \\
1690,1 \\
1934,7\end{array}$ & $\begin{array}{r}-379,0 \\
-182,0 \\
15,0\end{array}$ & $\begin{array}{l}-559,4 \\
-362,4 \\
-165,4\end{array}$ & $\begin{array}{l}-96,7 \\
100,3 \\
297,3\end{array}$ & $\begin{array}{l}254,2 \\
393,5 \\
532,8\end{array}$ & $\begin{array}{r}-53,5 \\
85,7 \\
225,0\end{array}$ & $\begin{array}{r}-79,1 \\
60,2 \\
199,5\end{array}$ & & $\begin{array}{l}614,7 \\
726,8 \\
838,9\end{array}$ & $\begin{array}{r}-67,3 \\
44,8 \\
156,9\end{array}$ \\
\hline 5 & $\begin{array}{l}2889,7 \\
3323,3 \\
3756,9\end{array}$ & $\begin{array}{l}-1089,4 \\
-740,1 \\
-390,8\end{array}$ & $\begin{array}{r}-253,5 \\
95,8 \\
445,1\end{array}$ & $\begin{array}{r}-418,1 \\
-68,8 \\
280,5\end{array}$ & $\begin{array}{r}-286,3 \\
-39,4 \\
207,5\end{array}$ & $\begin{array}{r}-215,6 \\
31,3 \\
278,2\end{array}$ & $\begin{array}{r}-238,8 \\
8,1 \\
255,0\end{array}$ & $\begin{array}{r}762,0 \\
960,7 \\
1159,4\end{array}$ & $\begin{array}{r}-112,2 \\
86,5 \\
285,2\end{array}$ & $\begin{array}{l}-89,9 \\
108,8 \\
307,5\end{array}$ \\
\hline 5.2 & $\begin{array}{l}2278,7 \\
2663,2 \\
3047,7\end{array}$ & $\begin{array}{r}-635,7 \\
-326,0 \\
-16,3\end{array}$ & $\begin{array}{r}-553,3 \\
-243,6 \\
66,1\end{array}$ & $\begin{array}{r}-154,3 \\
155,4 \\
465,1\end{array}$ & $\begin{array}{r}6,5 \\
225,4 \\
444,3\end{array}$ & $\begin{array}{r}-225,6 \\
-6,7 \\
212,2\end{array}$ & $\begin{array}{r}-168,3 \\
50,6 \\
269,5\end{array}$ & $\begin{array}{l}1021,2 \\
1197,4 \\
1373,6\end{array}$ & $\begin{array}{r}-92,9 \\
83,3 \\
259,5\end{array}$ & $\begin{array}{l}-43,9 \\
132,3 \\
308,5\end{array}$ \\
\hline 5.3 & $\begin{array}{l}1734,0 \\
1967,3 \\
2200,6\end{array}$ & $\begin{array}{l}-657,1 \\
-469,1 \\
-281,1\end{array}$ & $\begin{array}{l}-624,6 \\
-436,6 \\
-248,6\end{array}$ & $\begin{array}{l}-73,7 \\
114,3 \\
302,3\end{array}$ & $\begin{array}{r}49,9 \\
182,8 \\
315,7\end{array}$ & $\begin{array}{r}-182,5 \\
-49,6 \\
83,3\end{array}$ & $\begin{array}{r}-62,2 \\
70,7 \\
203,3\end{array}$ & $\begin{array}{l}237,2 \\
344,1 \\
451,0\end{array}$ & $\begin{array}{l}400,7 \\
507,6 \\
614,5\end{array}$ & $\begin{array}{r}-37,5 \\
69,4 \\
176,3\end{array}$ \\
\hline
\end{tabular}


125.

(continuação)

\begin{tabular}{|c|c|c|c|c|c|c|c|c|c|c|}
\hline idigo & $\hat{a}_{0}$ & $\hat{a}_{11}$ & $\tilde{a}_{22}$ & $\tilde{a}_{33}$ & $\hat{a}_{12}$ & $\hat{a}_{13}$ & $\hat{a}_{23}$ & $\hat{a}_{14}$ & $\tilde{a}_{24}$ & $\hat{a}_{34}$ \\
\hline & $\begin{array}{l}1722,4 \\
1955,7 \\
2189,0\end{array}$ & $\begin{array}{l}-635,9 \\
-447,9 \\
-259,9\end{array}$ & $\begin{array}{l}-673,5 \\
-485,5 \\
-297,5\end{array}$ & $\begin{array}{l}194,4 \\
382,4 \\
570,4\end{array}$ & $\begin{array}{l}139,2 \\
272,1 \\
405,0\end{array}$ & $\begin{array}{r}-134,0 \\
-1,1 \\
131,8\end{array}$ & $\begin{array}{r}-78,9 \\
54,0 \\
186,9\end{array}$ & $\begin{array}{l}502,0 \\
608,9 \\
715,8\end{array}$ & $\begin{array}{l}419,8 \\
526,7 \\
633,6\end{array}$ & $\begin{array}{r}98,8 \\
197,7\end{array}$ \\
\hline & $\begin{array}{l}1587,2 \\
1980,1 \\
2373,0\end{array}$ & $\begin{array}{r}-598,1 \\
-281,6 \\
34,9\end{array}$ & $\begin{array}{r}-618,3 \\
-301,8 \\
14,7\end{array}$ & $\begin{array}{l}153,4 \\
469,9 \\
786,4\end{array}$ & $\begin{array}{r}72 \\
296 \\
519\end{array}$ & $\begin{array}{r}-185 \\
38 \\
261\end{array}$ & $\begin{array}{l}2 \\
5 \\
2\end{array}$ & $\begin{array}{r}76 \\
94 \\
112\end{array}$ & $\begin{array}{l}289,9 \\
470,0 \\
650,1\end{array}$ & $\begin{array}{l}-49,8 \\
130,3 \\
310,4\end{array}$ \\
\hline & $\begin{array}{l}2316,8 \\
2613,5 \\
2910,2\end{array}$ & $\begin{array}{r}-530,7 \\
-291,7 \\
-52,7\end{array}$ & $\begin{array}{r}-294,4 \\
-55,4 \\
183,6\end{array}$ & $\begin{array}{r}-35 \\
-12 \\
11\end{array}$ & $\begin{array}{r}-282,4 \\
-113,4 \\
55,6\end{array}$ & $\begin{array}{r}-229,2 \\
-60,2 \\
108,8\end{array}$ & $\begin{array}{r}-10 \\
6 \\
23\end{array}$ & $\begin{array}{r}-103,6 \\
32,4 \\
168,5\end{array}$ & $\begin{array}{c}-182,3 \\
-46,3 \\
89,7\end{array}$ & $\begin{array}{r}-170,0 \\
-34,0 \\
102,0\end{array}$ \\
\hline & $\begin{array}{l}2070,2 \\
2435,3 \\
2800,4\end{array}$ & $\begin{array}{r}-259,4 \\
34,7 \\
328,8\end{array}$ & $\begin{array}{r}-374,8 \\
-80,7 \\
213,4\end{array}$ & $\begin{array}{r}-236,1 \\
58,0 \\
352,1\end{array}$ & $\begin{array}{r}-202,3 \\
5,6 \\
213,5\end{array}$ & $\begin{array}{r}-194,9 \\
13,0 \\
220,9\end{array}$ & & & $\begin{array}{r}-206,7 \\
-39,4 \\
127,9\end{array}$ & $\begin{array}{r}-214,1 \\
-46,8 \\
120,5\end{array}$ \\
\hline & $\begin{array}{r}932,8 \\
1249,9 \\
1576,0\end{array}$ & $\begin{array}{r}-401,6 \\
-138,9 \\
123,8\end{array}$ & $\begin{array}{l}-82,1 \\
180,6 \\
443,3\end{array}$ & $\begin{array}{r}-239,6 \\
23,1 \\
285,8\end{array}$ & $\begin{array}{r}-148,7 \\
37,0 \\
222,7\end{array}$ & $\begin{array}{r}-139,5 \\
46,2 \\
231,9\end{array}$ & $\begin{array}{r}-204,1 \\
-18,4 \\
167,3\end{array}$ & & $\begin{array}{r}-98,5 \\
51,0 \\
200,5\end{array}$ & $\begin{array}{r}-200,5 \\
-51,0 \\
98,5\end{array}$ \\
\hline & $\begin{array}{l}1424,6 \\
1768,4 \\
2112,2\end{array}$ & $\begin{array}{r}-341,0 \\
-64,0 \\
213,0\end{array}$ & $\begin{array}{r}-575,0 \\
-298,0 \\
-21,0\end{array}$ & $\begin{array}{r}-266,7 \\
10,3 \\
287,3\end{array}$ & $\begin{array}{r}-144,9 \\
50,9 \\
246,7\end{array}$ & $\begin{array}{r}-379,8 \\
-184,0 \\
11,8\end{array}$ & $\begin{array}{r}-170,3 \\
25,5 \\
221,3\end{array}$ & $\begin{array}{r}16,0 \\
173,6 \\
331,2\end{array}$ & $\begin{array}{l}206,5 \\
364,1 \\
521,7\end{array}$ & $\begin{array}{r}-153,6 \\
4,0 \\
161,6\end{array}$ \\
\hline & $\begin{array}{l}3503,0 \\
3942,4 \\
4381,8\end{array}$ & $\begin{array}{r}-505,7 \\
-151,7 \\
202,3\end{array}$ & $\begin{array}{l}-970,8 \\
-616,8 \\
-262,8\end{array}$ & $\begin{array}{l}-59,6 \\
294,4 \\
648,4\end{array}$ & $\begin{array}{l}-12,3 \\
237,9 \\
488,1\end{array}$ & $\begin{array}{r}-188,1 \\
62,1 \\
312,3\end{array}$ & & $\begin{array}{l}332,9 \\
534,3 \\
735,7\end{array}$ & $\begin{array}{r}629,6 \\
831,0 \\
1032,4\end{array}$ & $\begin{array}{r}-309,4 \\
-108,0 \\
93,4\end{array}$ \\
\hline 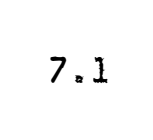 & $\begin{array}{l}2334,0 \\
2694,9 \\
3055,8\end{array}$ & $\begin{array}{r}-212,1 \\
78,6 \\
369,3\end{array}$ & $\begin{array}{r}-367,2 \\
-76,5 \\
214,2\end{array}$ & $\begin{array}{r}-209,9 \\
80,8 \\
371,5\end{array}$ & $\begin{array}{r}-388,3 \\
-182,8 \\
22,7\end{array}$ & $\begin{array}{r}-255,4 \\
-49,9 \\
155,6\end{array}$ & $\begin{array}{l}104,4 \\
309,9 \\
515,4\end{array}$ & $\begin{array}{r}-6,0 \\
159,4 \\
324,8\end{array}$ & $\begin{array}{r}-140,0 \\
25,4 \\
190,8\end{array}$ & $\begin{array}{r}-168,2 \\
-2,8 \\
162,6\end{array}$ \\
\hline 7.2 & $\begin{array}{l}3314,5 \\
3664,1 \\
4013,7\end{array}$ & $\begin{array}{r}-263,1 \\
18,5 \\
300,1\end{array}$ & $\begin{array}{r}-439,8 \\
-158,2 \\
123,4\end{array}$ & $\begin{array}{r}-120,3 \\
161,3 \\
442,9\end{array}$ & $\begin{array}{r}-99,9 \\
99,2 \\
298,3\end{array}$ & $\begin{array}{r}-259,8 \\
-60,7 \\
138,4\end{array}$ & $\begin{array}{r}-166,5 \\
32,6 \\
231,7\end{array}$ & $\begin{array}{l}605,1 \\
765,3 \\
925,5\end{array}$ & $\begin{array}{r}-149,9 \\
10,3 \\
170,5\end{array}$ & $\begin{array}{r}-301,1 \\
-140,9 \\
19,3\end{array}$ \\
\hline 8.1 & $\begin{array}{l}1403,7 \\
2137,9 \\
2872,3\end{array}$ & $\begin{array}{r}-963,4 \\
-371,8 \\
219,8\end{array}$ & $\begin{array}{r}-208,8 \\
283,8 \\
974,4\end{array}$ & $\begin{array}{r}-255,1 \\
366,5 \\
928,1\end{array}$ & $\begin{array}{r}-510,7 \\
-92,5 \\
325,7\end{array}$ & $\begin{array}{r}-360,5 \\
57,5 \\
475,9\end{array}$ & $\begin{array}{r}-608,0 \\
-189,8 \\
228,4\end{array}$ & $\begin{array}{r}-220,8 \\
115,8 \\
452,4\end{array}$ & $\begin{array}{r}-271,8 \\
64,8 \\
401,4\end{array}$ & $\begin{array}{r}-287,2 \\
49,4 \\
386,0\end{array}$ \\
\hline
\end{tabular}


126.

(continuaçẫo)

\begin{tabular}{|c|c|c|c|c|c|c|c|c|c|c|}
\hline idis & $\dot{a}_{0}$ & $\hat{a}_{11}$ & $\hat{a}_{22}$ & $\tilde{a}_{33}$ & $\hat{a}_{12}$ & $\hat{a}_{13}$ & $\hat{a}_{23}$ & $\hat{a}_{14}$ & $\hat{\mathrm{a}}_{24}$ & $\hat{a}_{34}$ \\
\hline 8.2 & $\begin{array}{l}3362 \\
3790 \\
4218\end{array}$ & $\begin{array}{l}-1005,9 \\
-661,1 \\
-316,3\end{array}$ & $\begin{array}{r}-412,6 \\
-67,8 \\
277,0\end{array}$ & $\begin{array}{r}-458,9 \\
-114,1 \\
230,7\end{array}$ & $\begin{array}{c}-38 \\
-15 \\
10\end{array}$ & $\begin{array}{r}-125,5 \\
118,2 \\
361,9\end{array}$ & $\begin{array}{l}.5 \\
.2 \\
.9\end{array}$ & $\begin{array}{l}220,7 \\
416,9 \\
613,1\end{array}$ & $\begin{array}{r}-137,5 \\
58,7 \\
254,9\end{array}$ & $\begin{array}{r}-254,9 \\
-58,7 \\
137,5\end{array}$ \\
\hline 8. & $\begin{array}{l}3282,2 \\
3818,3 \\
4354,4\end{array}$ & $\begin{array}{r}-423,2 \\
8,7 \\
440,6\end{array}$ & $\begin{array}{r}-426,9 \\
5,0 \\
436,9\end{array}$ & $\begin{array}{r}-17 \\
25 \\
69\end{array}$ & $\begin{array}{r}-296,0 \\
9,3 \\
314,6\end{array}$ & $\begin{array}{r}-319,3 \\
-14,0 \\
291,3\end{array}$ & $\begin{array}{r}-224,8 \\
80,5 \\
385,8\end{array}$ & $\begin{array}{r}-285,8 \\
-40,1 \\
205,6\end{array}$ & $\begin{array}{r}-364,9 \\
-119,2 \\
126,5\end{array}$ & $\begin{array}{r}-229,9 \\
15,8 \\
261,5\end{array}$ \\
\hline 8.4 & $\begin{array}{l}3753,4 \\
4402,6 \\
5051,8\end{array}$ & $\begin{array}{r}-773,4 \\
-250,4 \\
272,6\end{array}$ & $\begin{array}{r}-606,6 \\
-83,6 \\
439,4\end{array}$ & $\begin{array}{r}-862,3 \\
-339,3 \\
183,7\end{array}$ & $\begin{array}{r}-378,5 \\
-8,8 \\
360,9\end{array}$ & $\begin{array}{l}-14,4 \\
355,3 \\
725,0\end{array}$ & $\begin{array}{r}-236,8 \\
132,9 \\
502,6\end{array}$ & $\begin{array}{r}-170,1 \\
127,5 \\
425,1\end{array}$ & $\begin{array}{r}-225,5 \\
72,1 \\
369,7\end{array}$ & $\begin{array}{r}-503,5 \\
-205,9 \\
91,7\end{array}$ \\
\hline 8.5 & $\begin{array}{l}353 \\
413 \\
473\end{array}$ & $\begin{array}{l}-1166,3 \\
-682,2 \\
-198,1\end{array}$ & $\begin{array}{r}-650,8 \\
-166,7 \\
317,4\end{array}$ & $\begin{array}{r}-359,2 \\
-124,9 \\
359,2\end{array}$ & $\begin{array}{r}-21 \\
12 \\
46\end{array}$ & $\begin{array}{r}-105,9 \\
236,3 \\
578,5\end{array}$ & $\begin{array}{r}-326,8 \\
15,4 \\
357,6\end{array}$ & $\begin{array}{r}-257,2 \\
18,3 \\
293,8\end{array}$ & $\begin{array}{l}-95,6 \\
179,9 \\
455,4\end{array}$ & $\begin{array}{r}-151,6 \\
123,9 \\
339,4\end{array}$ \\
\hline 9.1 & $\begin{array}{l}1896,0 \\
2217,0 \\
2538,0\end{array}$ & $\begin{array}{l}-723,3 \\
-464,7 \\
-206,1\end{array}$ & $\begin{array}{l}-28.9 \\
229.7 \\
488,3\end{array}$ & $\begin{array}{r}-58 \\
-326 \\
-67\end{array}$ & $\begin{array}{r}-235,5 \\
-52,7 \\
130,1\end{array}$ & $\begin{array}{l}-13,3 \\
169,5 \\
352,3\end{array}$ & $\begin{array}{r}-363,5 \\
-180,7 \\
2,1\end{array}$ & $\begin{array}{l}525,4 \\
672,5 \\
819,6\end{array}$ & $\begin{array}{r}-272,9 \\
-125,8 \\
21,3\end{array}$ & $\begin{array}{r}8,7 \\
155,8 \\
302,9\end{array}$ \\
\hline 9.2 & $\begin{array}{l}1665,7 \\
2005,4 \\
2345,1\end{array}$ & $\begin{array}{r}-437,3 \\
-163,7 \\
109,9\end{array}$ & $\begin{array}{r}-154,7 \\
118,9 \\
392,5\end{array}$ & $\begin{array}{r}-441,3 \\
-168,3 \\
105,3\end{array}$ & $\begin{array}{r}-142,4 \\
51,0 \\
244,4\end{array}$ & $\begin{array}{l}-36,1 \\
157,3 \\
350,7\end{array}$ & $\begin{array}{r}-290,7 \\
-97,3 \\
96,1\end{array}$ & $\begin{array}{l}285,7 \\
441,4 \\
597,1\end{array}$ & $\begin{array}{r}-166,5 \\
-10,8 \\
144,9\end{array}$ & $\begin{array}{r}-120,2 \\
35,5 \\
191,2\end{array}$ \\
\hline 9.3 & $\begin{array}{l}2914,8 \\
3379,7 \\
3844,6\end{array}$ & $\begin{array}{r}-620,0 \\
-245,5 \\
129,0\end{array}$ & $\begin{array}{r}-610,6 \\
-236,1 \\
138,4\end{array}$ & $\begin{array}{l}-893,0 \\
-518,5 \\
-144,0\end{array}$ & $\begin{array}{r}-202,5 \\
62,3 \\
327,1\end{array}$ & $\begin{array}{r}-262,4 \\
2,4 \\
267,2\end{array}$ & $\begin{array}{r}-243,8 \\
21,0 \\
285,8\end{array}$ & $\begin{array}{l}1448,9 \\
1662,0 \\
1875,1\end{array}$ & $\begin{array}{r}-245,6 \\
-32,5 \\
180,6\end{array}$ & $\begin{array}{r}-225,4 \\
-12,3 \\
200,8\end{array}$ \\
\hline
\end{tabular}

Obs.: Para cada caso, a linha superior refere-se ao extremo inferior e a linha inferior ao extremo superior do intervalo de confiança do parâmetro em questão. 
Tabela $\theta$ - Estimativas do parämetro e respectivos intervalos de con fiança ao nível de $95 \%$ de probabilidade, das equações de superficie de resposta para os 9 grupos de ensaios fatoriais.

\begin{tabular}{|c|c|c|c|c|c|c|c|c|c|c|}
\hline Código & $\hat{a}_{0}$ & $\tilde{a}_{n}$ & $\hat{a}_{22}$ & $\hat{a}_{33}$ & $\bar{a}_{12}$ & $\hat{a}_{13}$ & $\hat{\mathrm{a}}_{23}$ & $\hat{a}_{14}$ & $\bar{a}_{24}$ & $\hat{a}_{34}$ \\
\hline 1 & $\begin{array}{l}1425,5 \\
1801,6 \\
2177,7\end{array}$ & $\begin{array}{l}-32,9 \\
270,1 \\
573,1\end{array}$ & $\begin{array}{r}-675,2 \\
-372,2 \\
-69,2\end{array}$ & $\begin{array}{r}89,8 \\
392,8 \\
695,8\end{array}$ & $\begin{array}{r}-340,2 \\
-126,0 \\
88,2\end{array}$ & $\begin{array}{r}-422,9 \\
-208,7 \\
5,5\end{array}$ & $\begin{array}{r}-192,8 \\
21,4 \\
235,6\end{array}$ & $\begin{array}{l}227,1 \\
399,5 \\
571,9\end{array}$ & $\begin{array}{r}-315,4 \\
-143,0 \\
29,4\end{array}$ & $\begin{array}{r}-214,1 \\
-41,7 \\
130,7\end{array}$ \\
\hline 2 & $\begin{array}{l}2210,8 \\
2388,8 \\
2566,8\end{array}$ & $\begin{array}{r}-316,8 \\
-173,4 \\
-30,0\end{array}$ & $\begin{array}{r}-296,4 \\
-153,0 \\
-9,6\end{array}$ & $\begin{array}{r}-150,5 \\
-7,1 \\
136,3\end{array}$ & $\begin{array}{r}59,2 \\
160,6 \\
262,0\end{array}$ & $\begin{array}{r}-117,6 \\
-16,2 \\
85,2\end{array}$ & $\begin{array}{r}28,3 \\
129,7 \\
231,1\end{array}$ & $\begin{array}{r}851,8 \\
933,4 \\
1015,0\end{array}$ & $\begin{array}{l}173,7 \\
255,3 \\
336,9\end{array}$ & $\begin{array}{r}-112,9 \\
-31,3 \\
50,3\end{array}$ \\
\hline 3 & $\begin{array}{l}2208,9 \\
2337,4 \\
2465,9\end{array}$ & $\begin{array}{l}-322,5 \\
-219,0 \\
-115,5\end{array}$ & $\begin{array}{r}-150,7 \\
-47,2 \\
56,3\end{array}$ & $\begin{array}{r}-4,4 \\
99,1 \\
202,6\end{array}$ & $\begin{array}{r}22,3 \\
95,5 \\
168,7\end{array}$ & $\begin{array}{r}-101,8 \\
-28,6 \\
44,6\end{array}$ & $\begin{array}{r}-95,7 \\
-22,5 \\
50,7\end{array}$ & $\begin{array}{l}338,8 \\
397,7 \\
456,6\end{array}$ & $\begin{array}{r}55,0 \\
113,9 \\
172,8\end{array}$ & $\begin{array}{r}-13,6 \\
45,3 \\
104,2\end{array}$ \\
\hline 4 & $\begin{array}{l}1799,9 \\
1992,6 \\
2185,3\end{array}$ & $\begin{array}{r}-336,4 \\
-181,4 \\
-26,2\end{array}$ & $\begin{array}{l}-476,0 \\
-320,8 \\
-165,6\end{array}$ & $\begin{array}{r}-195,5 \\
-40,3 \\
114,9\end{array}$ & $\begin{array}{l}200,5 \\
310,2 \\
419,9\end{array}$ & $\begin{array}{r}51,3 \\
161,0 \\
270,7\end{array}$ & $\begin{array}{r}-83,0 \\
26,7 \\
136,4\end{array}$ & $\begin{array}{l}447,8 \\
536,1 \\
624,4\end{array}$ & $\begin{array}{l}402,1 \\
490,4 \\
578,7\end{array}$ & $\begin{array}{r}-13,3 \\
75,0 \\
163,5\end{array}$ \\
\hline 5 & $\begin{array}{l}2223,1 \\
2377,9 \\
2532,7\end{array}$ & $\begin{array}{l}-577,6 \\
-452,9 \\
-328,2\end{array}$ & $\begin{array}{l}-399,0 \\
-274,3 \\
-149,6\end{array}$ & $\begin{array}{r}85,9 \\
210,6 \\
335,3\end{array}$ & $\begin{array}{r}96,3 \\
184,4 \\
272,5\end{array}$ & $\begin{array}{r}-85,7 \\
2,4 \\
90,5\end{array}$ & $\begin{array}{r}-28,5 \\
59,6 \\
147,7\end{array}$ & $\begin{array}{l}740,2 \\
811,1 \\
882,0\end{array}$ & $\begin{array}{l}263,9 \\
344,8 \\
405,7\end{array}$ & $\begin{array}{r}35,4 \\
106,3 \\
177,3\end{array}$ \\
\hline 6 & $\begin{array}{l}2242,0 \\
2401,9 \\
2561,8\end{array}$ & $\begin{array}{r}-251,1 \\
-122,3 \\
6,5\end{array}$ & $\begin{array}{r}-302,9 \\
-174,1 \\
-45,3\end{array}$ & $\begin{array}{r}-75,7 \\
53,1 \\
181,9\end{array}$ & $\begin{array}{r}-47,4 \\
43,6 \\
134,6\end{array}$ & $\begin{array}{r}-115,6 \\
-24,6 \\
66,4\end{array}$ & $\begin{array}{r}-14,9 \\
76,1 \\
167,1\end{array}$ & $\begin{array}{l}163,8 \\
237,1 \\
310,4\end{array}$ & $\begin{array}{l}158,8 \\
232,1 \\
305,4\end{array}$ & $\begin{array}{r}-120,5 \\
-47,2 \\
26,1\end{array}$ \\
\hline 7 & $\begin{array}{l}2928,3 \\
3179,5 \\
3430,7\end{array}$ & $\begin{array}{r}-153,9 \\
48,5 \\
250,9\end{array}$ & $\begin{array}{r}-319,7 \\
-117,3 \\
85,1\end{array}$ & $\begin{array}{l}-81,3 \\
121,1 \\
323,5\end{array}$ & $\begin{array}{r}-184,9 \\
-41,8 \\
101,3\end{array}$ & $\begin{array}{r}-198,4 \\
-55,3 \\
87,8\end{array}$ & $\begin{array}{r}28,2 \\
171,3 \\
314,4\end{array}$ & $\begin{array}{l}347,2 \\
462,3 \\
577,4\end{array}$ & $\begin{array}{r}-97,2 \\
17,9 \\
133,0\end{array}$ & $\begin{array}{r}-187,0 \\
71,9 \\
43,2\end{array}$ \\
\hline 8 & $\begin{array}{l}3388,5 \\
3656,3 \\
3924,1\end{array}$ & $\begin{array}{l}-607,1 \\
-391,4 \\
-175,7\end{array}$ & $\begin{array}{r}-201,8 \\
13,9 \\
229,6\end{array}$ & $\begin{array}{r}-212,1 \\
3,6 \\
219,3\end{array}$ & $\begin{array}{r}-173,8 \\
-21,3 \\
131,2\end{array}$ & $\begin{array}{r}-1,8 \\
150,7 \\
303,2\end{array}$ & $\begin{array}{r}-104,9 \\
47,6 \\
200,1\end{array}$ & $\begin{array}{r}5,0 \\
127,7 \\
250,4\end{array}$ & $\begin{array}{r}-71,4 \\
51,3 \\
174,0\end{array}$ & $\begin{array}{r}-137,8 \\
-15,1 \\
107,6\end{array}$ \\
\hline 9 & $\begin{array}{l}2314,3 \\
2534,0 \\
2753,7\end{array}$ & $\begin{array}{l}-468,3 \\
-291,3 \\
-114,3\end{array}$ & $\begin{array}{r}-139,5 \\
37,5 \\
214,5\end{array}$ & $\begin{array}{l}-514,6 \\
-337,6 \\
-160,6\end{array}$ & $\begin{array}{r}-104,9 \\
20,2 \\
145,3\end{array}$ & $\begin{array}{r}-15,4 \\
109,7 \\
234,8\end{array}$ & $\begin{array}{r}-210,8 \\
-85,7 \\
39,4\end{array}$ & $\begin{array}{r}824,6 \\
925,3 \\
1026,0\end{array}$ & $\begin{array}{r}-157,1 \\
-56,4 \\
44,3\end{array}$ & $\begin{array}{r}-41,0 \\
59,7 \\
160,4\end{array}$ \\
\hline
\end{tabular}

Obs.: Para cada caso, a Iinha superior refere-se ao extremo inferior e a linha inferior ao extremo superior do intervalo de confiança do parâmetro em questão. 
Tabela 9 - Estimativas dos parāmetros e respectivos desvios padrão do trinômio do segundo grau dos grupos de ensaios para o nitrogēnio, o fósforo e o potássio.

\begin{tabular}{|c|c|c|c|c|c|c|c|}
\hline Grupos & & à & $s(\hat{a})$ & 6 & $s(B)$ & $\overline{\mathrm{c}}$ & $s(\hat{c})$ \\
\hline 1 & $\begin{array}{l}N \\
P \\
K\end{array}$ & $\begin{array}{l}1815,3 \\
2243,5 \\
1733,5\end{array}$ & $\begin{array}{l}94,1 \\
95,1 \\
62,9\end{array}$ & $\begin{array}{r}399,5 \\
-143,0 \\
-41,7\end{array}$ & $\begin{array}{l}66,1 \\
66,8 \\
44,1\end{array}$ & $\begin{array}{r}270,1 \\
-372,2 \\
392,8\end{array}$ & $\begin{array}{r}116,1 \\
117,4 \\
77,6\end{array}$ \\
\hline 2 & $\begin{array}{l}N \\
P \\
K\end{array}$ & $\begin{array}{l}2282,1 \\
2268,4 \\
2171,2\end{array}$ & $\begin{array}{r}105,3 \\
48,1 \\
65,7\end{array}$ & $\begin{array}{l}933,4 \\
255,3 \\
-31,3\end{array}$ & $\begin{array}{l}74,0 \\
33,8 \\
46,1\end{array}$ & $\begin{array}{r}-173,4 \\
-153,0 \\
-7,1\end{array}$ & $\begin{array}{r}130,1 \\
59,4 \\
81,0\end{array}$ \\
\hline 3 & $\begin{array}{l}N \\
P \\
K\end{array}$ & $\begin{array}{l}2372,0 \\
2257,5 \\
2159,9\end{array}$ & $\begin{array}{l}55,9 \\
69,5 \\
50,9\end{array}$ & $\begin{array}{r}397,7 \\
113,9 \\
45,3\end{array}$ & $\begin{array}{l}39,3 \\
48,7 \\
35,8\end{array}$ & $\begin{array}{r}-219,0 \\
-47,2 \\
99,1\end{array}$ & $\begin{array}{l}69,0 \\
85,7 \\
62,9\end{array}$ \\
\hline 4 & $\begin{array}{l}N \\
P \\
K\end{array}$ & $\begin{array}{l}1751,9 \\
1844,4 \\
1657,8\end{array}$ & $\begin{array}{r}151,7 \\
247,4 \\
63,0\end{array}$ & $\begin{array}{r}536,1 \\
490,4 \\
75,0\end{array}$ & $\begin{array}{r}106,5 \\
173,7 \\
44,3\end{array}$ & $\begin{array}{r}-181,4 \\
-320,8 \\
-40,3\end{array}$ & $\begin{array}{r}187,3 \\
305,3 \\
77,7\end{array}$ \\
\hline 5 & $\begin{array}{l}N \\
P \\
K\end{array}$ & $\begin{array}{l}2335,4 \\
2216,4 \\
2033,5\end{array}$ & $\begin{array}{r}156,2 \\
117,9 \\
56,9\end{array}$ & $\begin{array}{l}811,1 \\
334,8 \\
106,3\end{array}$ & $\begin{array}{r}109,5 \\
82,8 \\
40,0\end{array}$ & $\begin{array}{r}-452,9 \\
-274,3 \\
70,2\end{array}$ & $\begin{array}{r}192,8 \\
145,5 \\
70,3\end{array}$ \\
\hline 6 & $\begin{array}{l}N \\
P \\
K\end{array}$ & $\begin{array}{l}2321,3 \\
2355,8 \\
2204,3\end{array}$ & $\begin{array}{r}87,5 \\
183,9 \\
42,8\end{array}$ & $\begin{array}{l}237,1 \\
232,1 \\
-47.2\end{array}$ & $\begin{array}{r}61,5 \\
129,1 \\
30,1\end{array}$ & $\begin{array}{r}-122,3 \\
-174,1 \\
53,1\end{array}$ & $\begin{array}{r}108,0 \\
226,8 \\
52,8\end{array}$ \\
\hline 7 & $\begin{array}{l}N \\
P \\
K\end{array}$ & $\begin{array}{l}3182,0 \\
3292,6 \\
3133,7\end{array}$ & $\begin{array}{r}302,5 \\
24,7 \\
72,7\end{array}$ & $\begin{array}{r}462,4 \\
17,9 \\
-71,9\end{array}$ & $\begin{array}{r}212,4 \\
17,3 \\
51,0\end{array}$ & $\begin{array}{r}48,5 \\
-117,3 \\
121,1\end{array}$ & $\begin{array}{r}373,3 \\
30,5 \\
89,7\end{array}$ \\
\hline 8 & $\begin{array}{l}N \\
P \\
K\end{array}$ & $\begin{array}{l}3667,9 \\
3397,7 \\
3404,6\end{array}$ & $\begin{array}{r}108,4 \\
73,3 \\
92,1\end{array}$ & $\begin{array}{r}127,7 \\
51,3 \\
-15,1\end{array}$ & $\begin{array}{l}76,1 \\
51,5 \\
64,7\end{array}$ & $\begin{array}{r}-391,4 \\
13,9 \\
3,6\end{array}$ & $\begin{array}{r}133,8 \\
90,4 \\
113,7\end{array}$ \\
\hline 9 & $\begin{array}{l}N \\
P \\
K\end{array}$ & $\begin{array}{l}2333,9 \\
2114,7 \\
2364,8\end{array}$ & $\begin{array}{r}376,8 \\
88,2 \\
76,7\end{array}$ & $\begin{array}{r}925,3 \\
-56,4 \\
59,7\end{array}$ & $\begin{array}{r}264,5 \\
61,9 \\
53,9\end{array}$ & $\begin{array}{r}-291,3 \\
37,5 \\
-337,6\end{array}$ & $\begin{array}{r}465,0 \\
108,9 \\
94,7\end{array}$ \\
\hline
\end{tabular}


129.

Tabela 10 - Estimativas dos parämetros e respectivos desvios padrão do trinômio do $2^{8}$ grau dos grupos de ensaios para o cal cário.

\begin{tabular}{ccccccc}
\hline Grupos & $\hat{a}$ & $s(\hat{a})$ & $\hat{6}$ & $s(\hat{b})$ & $\hat{c}$ & $s(\hat{c})$ \\
\hline 1 & 2861,1 & 139,1 & $-18,9$ & 98,5 & $-366,9$ & 170,4 \\
2 & 2594,9 & 66,0 & 28,9 & 46,7 & $-15,9$ & 80,9 \\
3 & 2520,0 & 62,7 & 106,4 & 44,4 & $-144,6$ & 76,8 \\
4 & 1834,3 & 73,4 & 18,7 & 52,0 & $-143,3$ & 90,0 \\
5 & 2412,0 & 46,3 & $-4,7$ & 32,8 & 32,2 & 56,7 \\
6 & 2569,8 & 65,5 & 59,9 & 46,4 & $-91,0$ & 80,2 \\
8 & 3698,2 & 130,4 & 93,5 & 92,4 & 4,6 & 159,8 \\
9 & 2541,2 & 145,7 & 188,1 & 103,2 & 70,6 & 178,5 \\
\hline
\end{tabular}


130.

Tabela 11 - Valores do teste $F$ das regressöes e desvios da regressão - coeficientes de determinação total dos 37 ensaios individuais e dos 9 grupos de ensaios fatoriais.

\begin{tabular}{|c|c|c|c|c|c|c|c|}
\hline \multirow[b]{2}{*}{ Cödigo } & \multicolumn{2}{|c|}{ Teste F } & \multirow[b]{2}{*}{$\begin{array}{l}R^{2} \\
(\%)\end{array}$} & \multirow[b]{2}{*}{ Código } & \multicolumn{2}{|c|}{ Teste F } & \multirow[b]{2}{*}{$\begin{array}{l}R^{2} \\
(\%)\end{array}$} \\
\hline & $\begin{array}{l}\text { Regres- } \\
\text { são }\end{array}$ & $\begin{array}{l}\text { Desvios } \\
\text { da Re- } \\
\text { gressão }\end{array}$ & & & $\begin{array}{l}\text { Regres- } \\
\text { são }\end{array}$ & $\begin{array}{l}\text { Desvios } \\
\text { da Re- } \\
\text { gressão }\end{array}$ & \\
\hline 1.1 & $2,39 \%$ & 1,57 & 47,65 & 7.1 & 1,91 & 0,94 & 54,91 \\
\hline 1.2 & $3,14^{\%}$ & 1,67 & 53,04 & 7.2 & $10,73 \% \%$ & 0,47 & 93,18 \\
\hline 2.1 & $13,75^{* \% *}$ & 0,64 & 92,79 & 8.1 & 0,71 & 0,73 & 36,69 \\
\hline 2.2 & $17,55^{* 3 \%}$ & 1,75 & 85,72 & 8.2 & $4,30 \% *$ & 1,63 & 61,37 \\
\hline 2.3 & $12,84^{2098}$ & 0,81 & 90,46 & 8.3 & 0,31 & 0,42 & 30,91 \\
\hline 2.4 & $14,00^{258 \%}$ & 0,85 & 90,78 & 8.4 & 1,09 & 0,87 & 42,76 \\
\hline 2.5 & $14,00^{26 \%}$ & 0,54 & 93,93 & 8.5 & 1,52 & 1,11 & 45,09 \\
\hline 3.1 & $3,23^{\circ}$ & 0,74 & 72,46 & 9.1 & $13,28^{j e * k}$ & $2,15 *$ & 78,73 \\
\hline 3.2 & $5,39^{\% * \%}$ & 0,93 & 77,66 & 9.2 & $4,38 \%$ & 1,09 & 70,54 \\
\hline 3.3 & $22,48^{* \% *}$ & 0,63 & 95,47 & 9.3 & $27,77 \%$ & 1,64 & 91,03 \\
\hline 3.4 & 2,01 & 1,78 & 40,32 & & & & \\
\hline 3.5 & 1,00 & 0,74 & 44,90 & $G_{1}$ & $2,52^{*}$ & 1,45 & 51,07 \\
\hline 3.6 & $12,68^{2 \% 2 \%}$ & 0,92 & 89,26 & $\mathrm{G}_{2}^{1}$ & $12,87 \% *$ & 0,34 & 95,77 \\
\hline & & & & & 4,19 & 0,28 & 90,03 \\
\hline 4.1 & $9,71^{80 \% 8}$ & $2,12^{*}$ & 73,23 & $G_{4}^{3}$ & $9,23 * *$ & 0,46 & 92,26 \\
\hline 4.2 & 10,14 & 0,79 & 88,49 & $G_{5}^{4}$ & $15,53 \%$ & 0,44 & 94,59 \\
\hline 4.3 & $14,14^{20.98}$ & 0,48 & 94,61 & $G_{G}^{5}$ & $2,17^{\%}$ & 0,33 & 79,68 \\
\hline 4.4 & $26,96^{3 \% *}$ & 1,03 & 93,98 & $G_{7}^{6}$ & $\begin{array}{l}4,16^{* 6 *} \\
0,50\end{array}$ & $\begin{array}{l}0,29 \\
0,13\end{array}$ & $\begin{array}{l}89,46 \\
69,57\end{array}$ \\
\hline 5.1 & $12,48 \%$ & 0,80 & 90,37 & $G_{9}^{8}$ & $13,49 *$ & 1,08 & 88,20 \\
\hline 5.2 & $21,84^{8030}$ & 0,93 & 93,38 & & & & \\
\hline $\begin{array}{l}5.3 \\
5.4\end{array}$ & $\begin{array}{l}20,92 \\
34,34 \% 8\end{array}$ & $\begin{array}{l}1,33 \\
3,09 * *\end{array}$ & $\begin{array}{l}90,44 \\
86,96\end{array}$ & \multirow{7}{*}{\multicolumn{4}{|c|}{$\begin{aligned} *= & \text { significativo ao nível de } \\
& 5 \% \text { de probabilidade. } \\
* & \text { significativo ao nível de } \\
& 1 \% \text { de probabilidade. }\end{aligned}$}} \\
\hline 5.5 & $17,84^{\%}$ & 0,88 & 92,36 & & & & \\
\hline 6.1 & 1,24 & 1,07 & 40,92 & & & & \\
\hline 6.2 & 1,00 & 1,33 & 31,09 & & & & \\
\hline 6.3 & 1,54, & 0,71 & 56,33 & & & & \\
\hline 6.4 & $3,83^{\% * \%}$ & 0,98 & 70,04 & & & & \\
\hline 6.5 & $13,02^{\% \% 8}$ & 1.74 & 81,77 & & & & \\
\hline
\end{tabular}


Tabola 12 - Produções observadas, suas estimativas e intervalos de confiança a $95 \%$ de probabilidade dos 27 tratamentos para os 9 grupos de ensaios fatoriais $\mathrm{em} \mathrm{kg} / \mathrm{ha}$.

\begin{tabular}{|c|c|c|c|c|c|c|c|c|}
\hline \multirow{3}{*}{$\begin{array}{l}\text { TRATA- } \\
\text { MENTOS } \\
000\end{array}$} & \multicolumn{4}{|c|}{ GRUPO 1} & \multicolumn{4}{|c|}{ GRUPO 2} \\
\hline & \multirow{2}{*}{$\frac{Y}{1251}$} & \multirow{2}{*}{$\frac{\hat{\gamma}}{1564}$} & \multicolumn{2}{|c|}{$\begin{array}{l}\text { Intervalos } \\
\text { de Confiança }\end{array}$} & \multirow{2}{*}{$\frac{Y}{1073}$} & \multirow{2}{*}{$\frac{8}{1172}$} & \multicolumn{2}{|c|}{$\begin{array}{l}\text { Intervalos } \\
\text { de Confiança }\end{array}$} \\
\hline & & & 1036 & 2092 & & & 922 & 1422 \\
\hline 001 & 1833 & 1317 & 883 & 1751 & 1274 & 1034 & 829 & 1239 \\
\hline 002 & 1764 & 1855 & 1327 & 2383 & 947 & 945 & 695 & 1195 \\
\hline 010 & 2694 & 1898 & 1370 & 2332 & 1070 & 1290 & 1085 & 1495 \\
\hline 011 & 1180 & 1672 & 1295 & 2048 & 1362 & 1282 & 1104 & 1460 \\
\hline 012 & 2567 & 2232 & 1798 & 2666 & 1237 & 1260 & 1055 & 1465 \\
\hline 020 & 1607 & 1487 & 959 & 2015 & 1245 & 1102 & 852 & 1352 \\
\hline 021 & 514 & 1283 & 849 & 1717 & 1358 & 1224 & 1019 & 1429 \\
\hline 022 & 1763 & 1864 & 1336 & 2392 & 1012 & 1331 & 1081 & 1581 \\
\hline 100 & 1625 & 2028 & 1594 & 2462 & 1995 & 2134 & 1929 & 2339 \\
\hline 101 & 1097 & 1572 & 1196 & 1948 & 1870 & 1981 & 1803 & 2159 \\
\hline 102 & 2167 & 1902 & 1468 & 2336 & 1765 & 1812 & 1607 & 2017 \\
\hline 110 & 2070 & 2236 & 1860 & 2612 & 2490 & 2413 & 2235 & 2591 \\
\hline 111 & 1889 & 1802 & 3425 & 2178 & 2348 & 2389 & 2211 & 2567 \\
\hline 112 & 1736 & 2153 & 1777 & 2529 & 2412 & 2350 & 2172 & 2528 \\
\hline 120 & 1805 & 1699 & 1265 & 2133 & 2501 & 2386 & 2181 & 2591 \\
\hline 121 & 1685 & 1286 & 910 & 1662 & 2331 & 2491 & 2313 & 2669 \\
\hline 122 & 2263 & 1659 & 1225 & 2093 & 2826 & 2582 & 2377 & 2787 \\
\hline 200 & 3850 & 3033 & 2505 & 3561 & 2929 & 2750 & 2500 & 3000 \\
\hline 201 & 1708 & 2368 & 1934 & 2802 & 2612 & 2580 & 2375 & 2785 \\
\hline 202 & 2833 & 2489 & 1961 & 3017 & 2276 & 2396 & 2146 & 2646 \\
\hline 210 & 2499 & 3114 & 2680 & 3548 & 3129 & 3189 & 2984 & 3394 \\
\hline 211 & 3681 & 2471 & 2095 & 2847 & 3429 & 3149 & 2791 & 3327 \\
\hline 212 & 1875 & 2614 & 2180 & 3048 & 2940 & 3094 & 2889 & 3299 \\
\hline 220 & 2111 & 2452 & 1924 & 2980 & 3326 & 3322 & 3072 & 3572 \\
\hline 221 & 2014 & 1931 & 1497 & 2365 & 2956 & 3412 & 3207 & 3617 \\
\hline 222 & 1791 & 1994 & 1466 & 2522 & 3782 & 3487 & 3237 & 3737 \\
\hline
\end{tabular}

$Y=$ Produção observada;

$\varphi=$ Produção estimada. - 
(contInuação)

\begin{tabular}{|c|c|c|c|c|c|c|c|c|}
\hline \multirow{3}{*}{$\begin{array}{l}\text { TRATA- } \\
\text { MENTOS } \\
\text { rOO }\end{array}$} & \multicolumn{4}{|c|}{ GRUPO 3} & \multicolumn{4}{|c|}{ GRUPO 4} \\
\hline & \multirow{2}{*}{$\frac{Y}{1646}$} & \multirow{2}{*}{$\frac{\hat{Y}}{1658}$} & \multicolumn{2}{|c|}{$\begin{array}{l}\text { Intervalos } \\
\text { de Confiança }\end{array}$} & \multirow{2}{*}{$\frac{Y}{808}$} & \multirow{2}{*}{$\frac{9}{847}$} & \multicolumn{2}{|c|}{$\begin{array}{l}\text { Intervalos } \\
\text { de Confiança }\end{array}$} \\
\hline & & & 1478 & 1838 & & & 577 & 1117 \\
\hline 001 & 1688 & 1655 & 1507 & 1803 & 795 & 774 & 552 & 996 \\
\hline 002 & 1915 & 1851 & 1671 & 2031 & 786 & 621 & 351 & 891 \\
\hline 010 & 1723 & 1746 & 1598 & 1894 & 1064 & 1321 & 1099 & 1591 \\
\hline 011 & 1646 & 1721 & 1593 & 1849 & 1355 & 1275 & 1082 & 1468 \\
\hline 012 & 1881 & 1894 & 1746 & 2042 & 966 & 1149 & 927 & 1371 \\
\hline 020 & 1761 & 1740 & 1560 & 1920 & 1189 & 1153 & 883 & 1423 \\
\hline 021 & 1611 & 1692 & 1544 & 1840 & 1072 & 1135 & 913 & 1357 \\
\hline 022 & 1927 & 1842 & 1662 & 2022 & 1275 & 1035 & 765 & 1305 \\
\hline 100 & 2149 & 2208 & 2060 & 2356 & 1356 & 1093 & 871 & 1315 \\
\hline 101 & 2186 & 2176 & 2039 & 2304 & 810 & 1181 & 988 & 1374 \\
\hline 102 & 2334 & 2343 & 2195 & 2491 & 1207 & 1189 & 967 & 1411 \\
\hline 110 & 2659 & 2391 & 2263 & 2519 & 2198 & 1877 & 1694 & 2070 \\
\hline 111 & 1989 & 2337 & 2209 & 2465 & 1771 & 1993 & 1800 & 2180 \\
\hline 112 & 2561 & 2482 & 2354 & 2610 & 2244 & 2027 & 1834 & 2220 \\
\hline 120 & 2586 & 2481 & 2333 & 2629 & 2232 & 2020 & 1798 & 2242 \\
\hline 121 & 2414 & 2404 & 2276 & 2532 & 2121 & 2162 & 1969 & 2355 \\
\hline 122 & 2471 & 2526 & 2378 & 2674 & 1826 & 2224 & 2002 & 2446 \\
\hline 200 & 2218 & 2319 & 2139 & 2499 & 845 & 976 & 705 & 1246 \\
\hline 201 & 2357 & 2259 & 2111 & 2407 & 1292 & 1226 & 1004 & 1448 \\
\hline 202 & 2375 & 2398 & 2218 & 2578 & 1404 & 1395 & 1125 & 1665 \\
\hline 210 & 2607 & 2599 & 2451 & 2747 & 2067 & 2071 & 1849 & 2293 \\
\hline 211 & 2791 & 2516 & 2388 & 2644 & 2564 & 2347 & 2154 & 2540 \\
\hline 212 & 2461 & 2632 & 2484 & 2780 & 2375 & 2543 & 2321 & 2765 \\
\hline 220 & 2575 & 2783 & 2603 & 2963 & 2121 & 2524 & 2254 & 2794 \\
\hline 221 & 2758 & 2678 & 2530 & 2826 & 3141 & 2827 & 2605 & 3049 \\
\hline 222 & 2814 & 2772 & 2592 & 2952 & 3151 & 3049 & 2779 & 3319 \\
\hline
\end{tabular}


133.

(continuação)

\begin{tabular}{|c|c|c|c|c|c|c|c|c|}
\hline \multirow{3}{*}{$\begin{array}{l}\text { TRATA- } \\
\text { MENTOS } \\
000\end{array}$} & \multicolumn{4}{|c|}{ GRUPO 5} & \multicolumn{4}{|c|}{ GRUPO 6} \\
\hline & \multirow{2}{*}{$\frac{Y}{903}$} & \multirow{2}{*}{$\begin{array}{c}\hat{Y} \\
855\end{array}$} & \multicolumn{2}{|c|}{$\begin{array}{l}\text { Intervalos } \\
\text { de Confiança }\end{array}$} & \multirow{2}{*}{$\begin{array}{r}Y \\
1898\end{array}$} & \multirow{2}{*}{$\begin{array}{c}\gamma \\
1832\end{array}$} & \multicolumn{2}{|c|}{$\begin{array}{l}\text { Intervalos } \\
\text { de Confiança }\end{array}$} \\
\hline & & & 633 & 1072 & & & 1608 & 2056 \\
\hline 001 & 882 & 689 & 511 & 867 & 1943 & 1680 & 1496 & 1864 \\
\hline 002 & 900 & 944 & 727 & 1161 & 1682 & 1634 & 1410 & 1858 \\
\hline 010 & 1089 & 1221 & 1043 & 1399 & 1893 & 2118 & 1934 & 2302 \\
\hline 011 & 880 & 1114 & 959 & 1269 & 1846 & 2043 & 1883 & 2203 \\
\hline 012 & 1444 & 1428 & 1250 & 1606 & 1904 & 2024 & 1840 & 2208 \\
\hline 020 & 976 & 1037 & 820 & 1254 & 2137 & 2057 & 1833 & 2281 \\
\hline 021 & 1584 & 990 & 812 & 1168 & 2221 & 2057 & 1873 & 2241 \\
\hline 022 & 1005 & 1364 & 1147 & 1581 & 2132 & 2163 & 1939 & 2387 \\
\hline 100 & 1812 & 1933 & 1755 & 2111 & 2026 & 2172 & 1988 & 2356 \\
\hline 101 & 1906 & 1769 & 1614 & 1924 & 1704 & 1996 & 1836 & 2156 \\
\hline 102 & 1997 & 2026 & 1848 & 2204 & 1977 & 1925 & 1741 & 2109 \\
\hline 110 & 2494 & 2482 & 2327 & 2637 & 2854 & 2502 & 2342 & 2662 \\
\hline 111 & 2347 & 2378 & 2223 & 2533 & 2393 & 2402 & 2242 & 2562 \\
\hline 112 & 2769 & 2695 & 2540 & 2850 & 2509 & 2408 & 2248 & 2568 \\
\hline 120 & 2322 & 2483 & 2305 & 2661 & 2426 & 2484 & 2300 & 2668 \\
\hline 121 & 2350 & 2438 & 2283 & 2593 & 2315 & 2460 & 2300 & 2620 \\
\hline 122 & 3021 & 2815 & 2637 & 2993 & 2687 & 2542 & 2358 & 2726 \\
\hline 200 & 2110 & 2104 & 1887 & 2321 & 2243 & 2268 & 2044 & 2492 \\
\hline 201 & 1585 & 1943 & 1765 & 2121 & 2087 & 2067 & 1883 & 2251 \\
\hline 202 & 2389 & 2202 & 1985 & 2419 & 1985 & 1972 & 1748 & 2196 \\
\hline 210 & 3237 & 2838 & 2660 & 3016 & 2693 & 2642 & 2458 & 2826 \\
\hline 211 & 2565 & 2736 & 2581 & 2891 & 2798 & 2517 & 2357 & 2667 \\
\hline 212 & 2922 & 3055 & 2877 & 3233 & 2312 & 2498 & 2314 & 2682 \\
\hline 220 & 3033 & 3023 & 2806 & 3240 & 2571 & 2667 & 2443 & 2891 \\
\hline 221 & 2739 & 2981 & 2803 & 3159 & 2531 & 2618 & 2434 & 2802 \\
\hline 222 & 3461 & 3360 & 3143 & 3577 & 2704 & 2676 & 2452 & 2900 \\
\hline
\end{tabular}


(continuação)

\begin{tabular}{|c|c|c|c|c|c|c|c|c|}
\hline \multirow{3}{*}{$\begin{array}{l}\text { TRATA- } \\
\text { MENTOS } \\
000\end{array}$} & \multicolumn{4}{|c|}{ GRUPO 7} & \multicolumn{4}{|c|}{ GRUPO 8} \\
\hline & \multirow{2}{*}{$\begin{array}{c}Y \\
2968\end{array}$} & \multirow{2}{*}{$\begin{array}{c}P \\
2898 \\
\end{array}$} & \multicolumn{2}{|c|}{$\begin{array}{l}\text { Intervalos } \\
\text { de Confiança }\end{array}$} & \multirow{2}{*}{$\begin{array}{c}\gamma \\
3318\end{array}$} & \multirow{2}{*}{$\begin{array}{r}9 \\
3295 \\
\end{array}$} & \multicolumn{2}{|c|}{$\begin{array}{l}\text { Intervalos } \\
\text { de Confiança }\end{array}$} \\
\hline & & & 2546 & 3250 & & & 2919 & 3671 \\
\hline 001 & 2731 & 2589 & 2299 & 2879 & 3113 & 3078 & 2769 & 3387 \\
\hline 002 & 2393 & 2522 & 2170 & 2874 & 2798 & 2869 & 2493 & 3245 \\
\hline 010 & 2765 & 2903 & 2613 & 3193 & 3040 & 3307 & 2998 & 3616 \\
\hline 011 & 2658 & 2766 & 2515 & 3017 & 3362 & 3137 & 2869 & 3405 \\
\hline 012 & 3085 & 2870 & 2580 & 3160 & 3091 & 2975 & 2666 & 3284 \\
\hline 020 & 2769 & 2675 & 2323 & 3027 & 3137 & 3345 & 2969 & 3721 \\
\hline 021 & 2531 & 2708 & 2418 & 2998 & 3604 & 3224 & 2915 & 3533 \\
\hline 022 & 2985 & 2984 & 2632 & 3336 & 2877 & 3109 & 2733 & 3485 \\
\hline 100 & 3346 & 3409 & 3119 & 3699 & 3770 & 3685 & 3376 & 3994 \\
\hline 101 & 3174 & 3044 & 2793 & 3295 & 3549 & 3619 & 3351 & 3887 \\
\hline 102 & 2869 & 2922 & 2632 & 3212 & 3396 & 3560 & 3251 & 3869 \\
\hline 110 & 3243 & 3373 & 3122 & 3624 & 3774 & 3675 & 3407 & 3943 \\
\hline 111 & 3086 & 3179 & 2928 & 3430 & 3759 & 3656 & 3388 & 3924 \\
\hline 112 & 3091 & 3229 & 2978 & 3480 & 3649 & 3645 & 3377 & 3913 \\
\hline 120 & 3451 & 3102 & 2812 & 3392 & 3870 & 3693 & 3384 & 4002 \\
\hline 121 & 2914 & 3080 & 2829 & 3331 & 3537 & 3721 & 3453 & 3989 \\
\hline 122 & 3464 & 3301 & 3011 & 3591 & 3723 & 3758 & 3449 & 4067 \\
\hline 200 & 3886 & 4017 & 3665 & 4369 & 3349 & 3292 & 2916 & 3668 \\
\hline 201 & 3671 & 3597 & 3307 & 3887 & 3254 & 3377 & 3068 & 3686 \\
\hline 202 & 3379 & 3530 & 3178 & 3882 & 3496 & 3468 & 3092 & 3844 \\
\hline 210 & 4197 & 3939 & 3649 & 4229 & 3302 & 3261 & 2952 & 3570 \\
\hline 211 & 3710 & 3690 & 3439 & 3941 & 2978 & 3393 & 3125 & 3661 \\
\hline 212 & 3767 & 3684 & 3394 & 3974 & 3620 & 3532 & 3223 & 3841 \\
\hline 220 & 3316 & 3626 & 3274 & 3978 & 3271 & 37.57 & 2881 & 3633 \\
\hline 221 & 3729 & 3549 & 3259 & 3839 & 3481 & 3437 & 3128 & 3746 \\
\hline 222 & 3582 & 3714 & 3362 & 4066 & 3682 & 3623 & 3247 & 3999 \\
\hline
\end{tabular}


135.

(continuação)

\begin{tabular}{|c|c|c|c|c|}
\hline \multirow{2}{*}{$\begin{array}{l}\text { TRATA- } \\
\text { MENTOS }\end{array}$} & \multicolumn{4}{|c|}{ GRUPO 9} \\
\hline & $Y$ & $\bar{\gamma}$ & $\begin{array}{l}\text { Inte } \\
\text { de } \mathrm{Co}\end{array}$ & $\begin{array}{l}\text { alos } \\
\text { iança }\end{array}$ \\
\hline 000 & 767 & 1058 & 750 & 1366 \\
\hline 001 & 1787 & 1431 & 1178 & 1684 \\
\hline 002 & 989 & 1130 & 822 & 1438 \\
\hline 010 & 898 & 1030 & 777 & 1283 \\
\hline 011 & 2195 & 1317 & 1097 & 1537 \\
\hline 012 & 715 & 930 & 677 & 1183 \\
\hline 020 & 941 & 1076 & 767 & 1384 \\
\hline 021 & 921 & 1278 & 1025 & 1531 \\
\hline 022 & 843 & 805 & 497 & 1113 \\
\hline 100 & 2333 & 2145 & 1892 & 2398 \\
\hline 101 & 2639 & 2628 & 2408 & 2848 \\
\hline 102 & 1985 & 2436 & 2183 & 2689 \\
\hline 110 & 1835 & 2137 & 1917 & 2357 \\
\hline 111 & 2422 & 2534 & 2314 & 2754 \\
\hline 112 & 2413 & 2256 & 2036 & 2476 \\
\hline 120 & 2780 & 2203 & 1950 & 2456 \\
\hline 121 & 2190 & 2515 & 2295 & 2735 \\
\hline 122 & 2406 & 2151 & 1898 & 2404 \\
\hline 200 & 2564 & 2649 & 2341 & 2957 \\
\hline 201 & 3369 & 3242 & 2989 & 3495 \\
\hline 202 & 3445 & 3159 & 2851 & 3467 \\
\hline 210 & 2886 & 2661 & 2408 & 2914 \\
\hline 211 & 2621 & 3168 & 2948 & 3388 \\
\hline 212 & 3046 & 3000 & 2747 & 3253 \\
\hline 220 & 2713 & 2748 & 2440 & 3056 \\
\hline 221 & 3139 & 3169 & 2916 & 3422 \\
\hline 222 & 2928 & 2915 & 2607 & 3223 \\
\hline
\end{tabular}


Tabele 13 - Soluçōes e discussões dos sistemas de equações obtidas das derivadas parciais de primeira ordem das funções de receita liquida, para os 9 grupos de ensaios fatoriais.

\begin{tabular}{ccccccccc}
\hline \multirow{2}{*}{ GRUPOS } & \multicolumn{2}{c}{ Solução $(\mathrm{kg} / \mathrm{ha})$} & $\Delta_{1}$ & $\Delta_{2} / \Delta_{1}$ & $\Delta_{3} / \Delta_{2}$ & Ponto \\
\cline { 2 - 6 } & \multicolumn{1}{c}{$x_{1}^{+}$} & $x_{2}^{+}$ & $x_{3}^{+}$ & & & \\
\hline 1 & 43,1 & 18,9 & 34,0 & 540,2 & $-773,8$ & 705,9 & Sela \\
3 & 155,1 & 135,1 & 70,9 & $-346,8$ & $-231,6$ & 51,0 & Sela \\
4 & 14,3 & $-151,7$ & 19,8 & $-438,0$ & $-73,7$ & 211,3 & Sela \\
5 & $-11,5$ & 27,3 & $-50,2$ & $-362,8$ & $-376,4$ & 62,6 & Sela \\
6 & 14,3 & 63,7 & 27,6 & $-905,9$ & $-509,8$ & 428,3 & Sela \\
7 & 11,5 & 43,3 & 66,0 & $-244,6$ & $-340,4$ & 123,6 & Sela \\
8 & 156,9 & $-337,1$ & 386,1 & $-782,8$ & 28,4 & $-30,5$ & Sela \\
9 & 100,5 & 363,6 & 14,9 & $-582,6$ & 75,6 & $-743,1$ & Sela \\
\hline
\end{tabular}


Tabela 14 - Cortes da superfície - Doses ätimas de $\mathrm{N}, \mathrm{P}_{2} \mathrm{O}_{5}$ e $\mathrm{K}_{2} \mathrm{O}$ e respectivos intervalos de conflança ao nível de $95 \%$ de probabilidade para os 9 grupos de ensaios fatorials.

\begin{tabular}{|c|c|c|c|}
\hline GRUPOS & $\begin{array}{c}N \\
(\mathrm{~kg} / \mathrm{ha})\end{array}$ & $\begin{array}{c}\mathrm{P}_{2} \mathrm{O}_{5} \\
(\mathrm{~kg} / \mathrm{ha})\end{array}$ & $\begin{array}{c}\mathrm{K}_{2} \mathrm{O} \\
(\mathrm{kg} / \mathrm{ha})\end{array}$ \\
\hline 1 & minimo & $\begin{array}{r}-20,0 \\
17,3 \\
54,6\end{array}$ & mínimo \\
\hline 2 & $\begin{array}{r}62,9 \\
129,3 \\
195,7\end{array}$ & $\begin{array}{r}5,2 \\
34,2 \\
63,2\end{array}$ & $\begin{array}{r}-4825,1 \\
-199,9 \\
4425,3\end{array}$ \\
\hline 3 & $\begin{array}{l}44,7 \\
51,6 \\
58,5\end{array}$ & $\begin{array}{r}-494,2 \\
-113,5 \\
267,2\end{array}$ & mínimo \\
\hline 4 & $\begin{array}{l}49,3 \\
71,0 \\
92,7\end{array}$ & $\begin{array}{l}60,1 \\
69,7 \\
79,2\end{array}$ & $\begin{array}{l}-4,4 \\
29,0 \\
62,5\end{array}$ \\
\hline 5 & $\begin{array}{l}66,0 \\
73,6 \\
81,2\end{array}$ & $\begin{array}{l}46,1 \\
54,3 \\
62,5\end{array}$ & mínimo \\
\hline 6 & $\begin{array}{r}-14,7 \\
20,1 \\
54,9\end{array}$ & $\begin{array}{l}10,0 \\
33,4 \\
56,8\end{array}$ & mínimo \\
\hline 7 & minimo & $\begin{array}{r}-200,1 \\
-34,3 \\
131,5\end{array}$ & mínimo \\
\hline 8 & $\begin{array}{l}21,7 \\
33,7 \\
45,7\end{array}$ & mínimo & mínimo \\
\hline 9 & $\begin{array}{r}67,1 \\
96,5 \\
125,9\end{array}$ & mínimo & $\begin{array}{l}24,7 \\
29,2 \\
33,7\end{array}$ \\
\hline
\end{tabular}

Obs.: Para cada caso, a linha superior refere-se ao extremo inferior e a linha inferior ao extremo superior do intervalo de confian ça da dose em questão. 
138.

Tabela 15 - Doses ótimas de calcário pelo trinômio do segundo grau e respectivos intervalos de confiança ao nível de $95 \%$ de probabilidade para os grupos de ensaios dos tratamen tos adicionais.

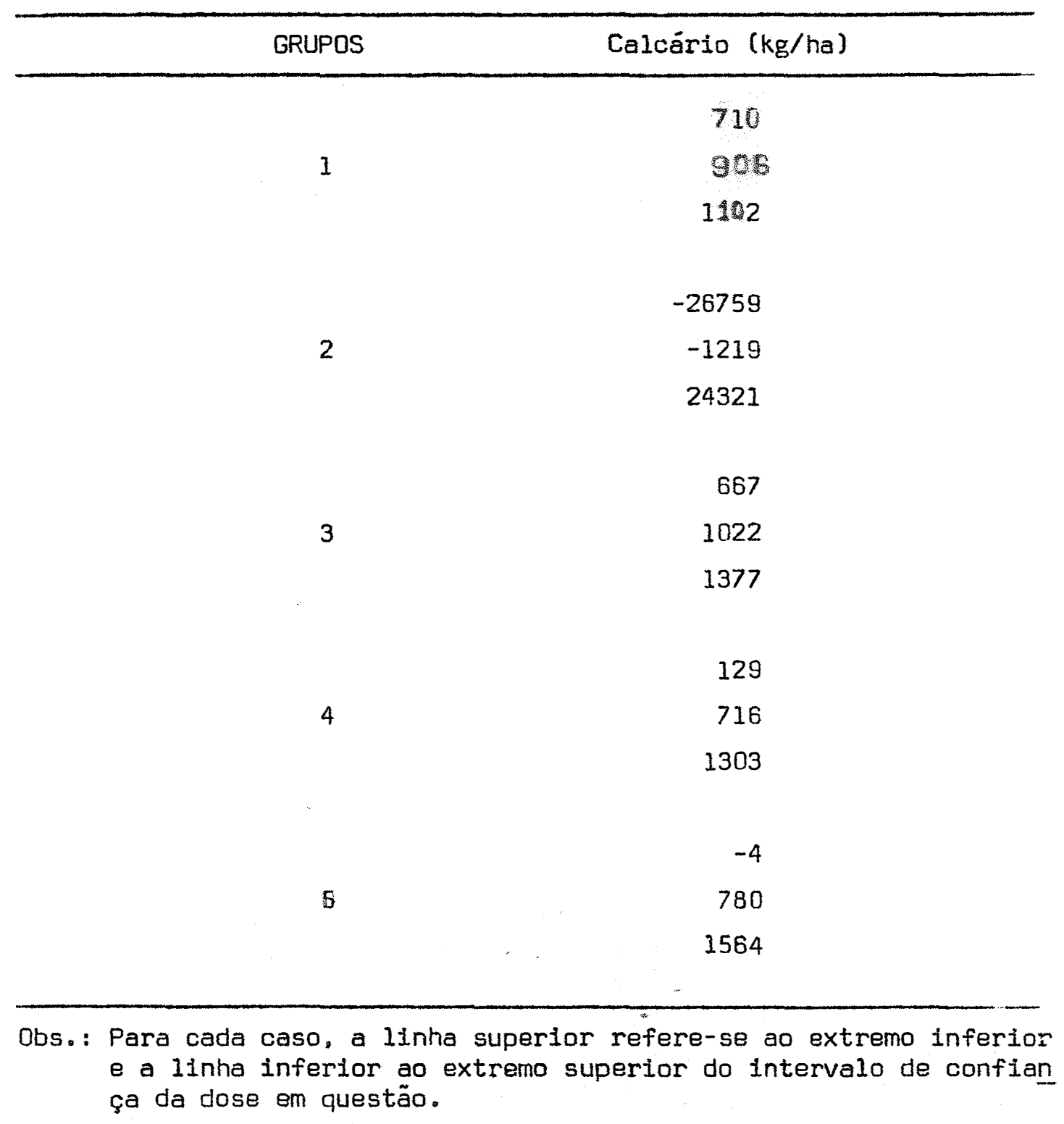

